\title{
Evaluation of Models and Data for Assessing Whooping Crane Habitat in the Central Platte River, Nebraska
}

Scientific Investigations Report 2005-5123

U.S. Department of the Interior

U.S. Geological Survey 


\section{Evaluation of Models and Data for Assessing Whooping Crane Habitat in the Central Platte River, Nebraska}

By Adrian H. Farmer, Brian S. Cade, James W. Terrell, and Jim H. Henriksen, U.S.

Geological Survey, and Jeffery T. Runge, U.S. Fish and Wildlife Service

Scientific Investigations Report 2005-5123 


\section{U.S. Department of the Interior \\ GALE A. NORTON, Secretary \\ U.S. Geological Survey \\ Charles G. Groat, Director}

U.S. Geological Survey, Reston, Virginia: 2005

For more information about the USGS and its products:

Telephone: 1-888-ASK-USGS

World Wide Web: http://www.usgs.gov/

Any use of trade, product, or firm names in this publication is for descriptive purposes only and does not imply endorsement by the U.S. Government.

Although this report is in the public domain, it contains copyrighted materials that are noted in the text.

Permission to reproduce those items must be secured from the individual copyright owners.

Suggested citation:

Farmer, A.H., Cade, B.S., Terrell, J.W., Henriksen, J.H., and Runge, J.T., 2005, Evaluation of models and data for assessing Whooping Crane habitat in the Central Platte River, Nebraska: U.S. Geological Survey, Biological Resources Discipline, Scientific Investigations Report 2005-5123, 64 p.

This report is available online at:

http://www.fort.usgs.gov/products/publications/21401/21401.asp 


\section{Contents}

Executive Summary .........................................................................................................................

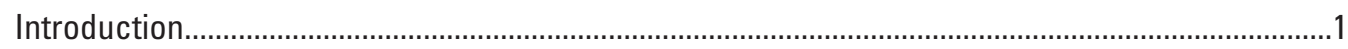

Historical Overview of Habitat Model Development ............................................................

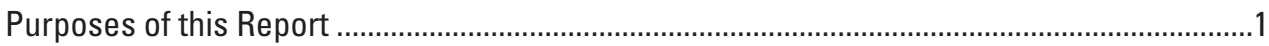

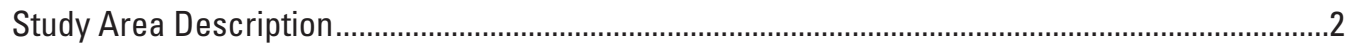

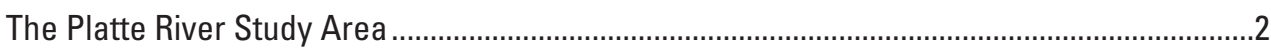

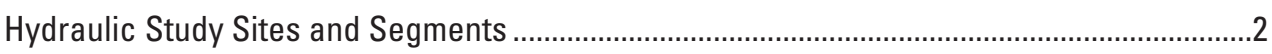

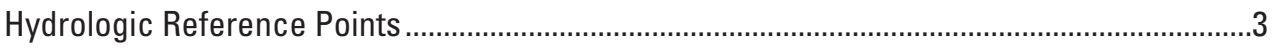

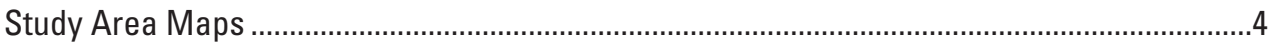

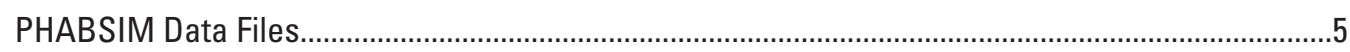

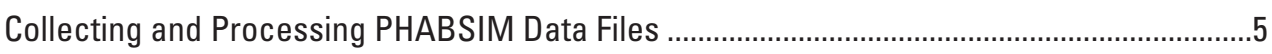

Land Cover Codes Used Before 1998 .............................................................................

Land Cover Codes for the 1998-2004 Cross Sections ...........................................................

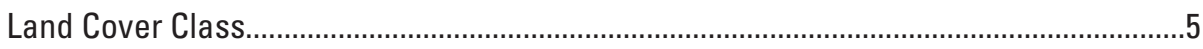

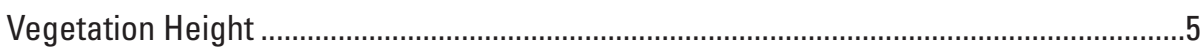

Vegetation Management ......................................................................................

Completeness, Adequacy, and Accuracy of the IFG4 Data Files............................................6

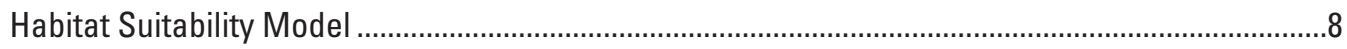

Habitat Model Development History .............................................................................

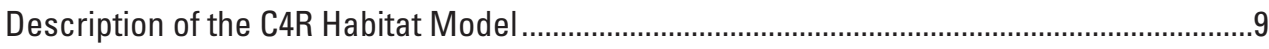

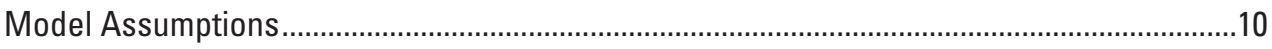

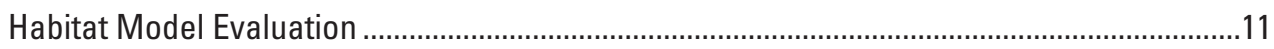

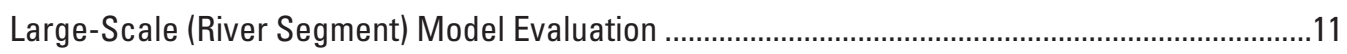

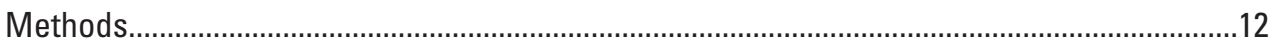

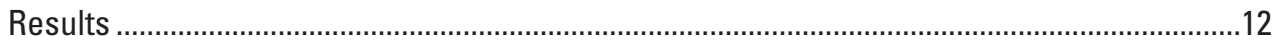

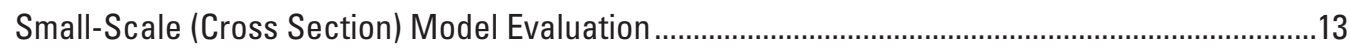

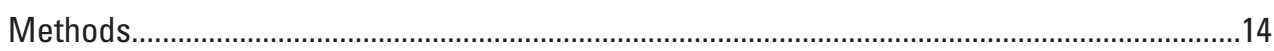

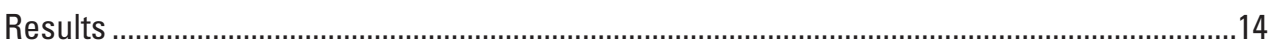

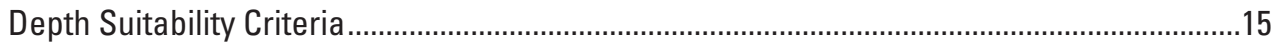

A Mathematical Bias toward High Flows ........................................................................

Alternative Depth Functions ...............................................................................................17

Comparing Depth Criteria Based on Crane Observations ......................................................17

An Evaluation of Crane Roost Observations versus Discharge ....................................................19

An Alternative to the Habitat Model ............................................................................... 19

Methods and Results.................................................................................................19

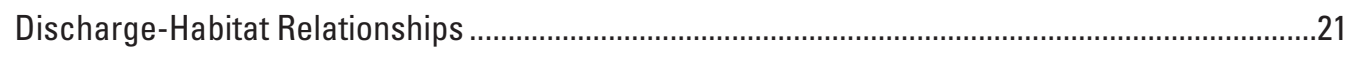

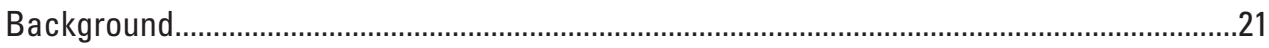

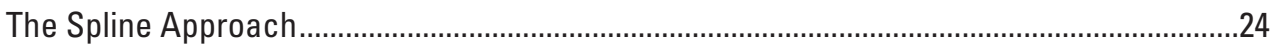

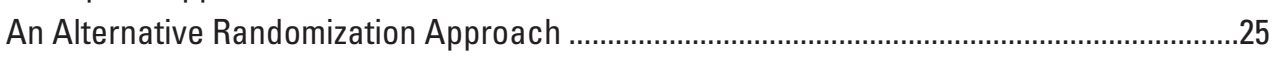

Example 1. Estimating the Discharge-Habitat Relationships for a Single

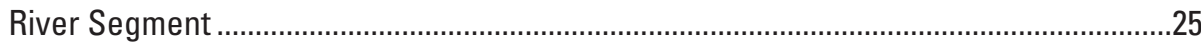

Example 2. Estimating the Optimal Flow forMultiple River Segments .............................25 
Computer Programs

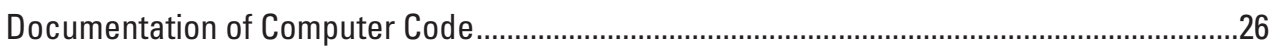

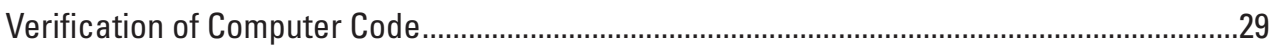

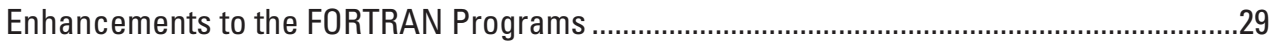

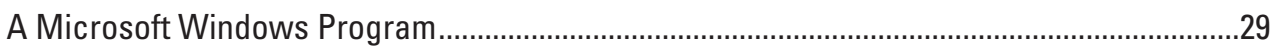

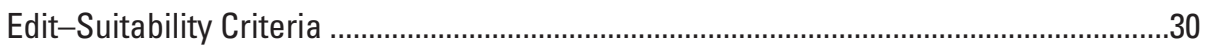

Edit-Segment Lengths ........................................................................................

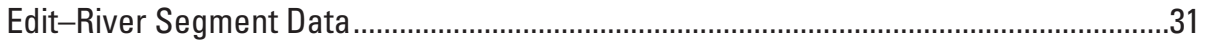

Analyses-Optimum Flow .......................................................................................

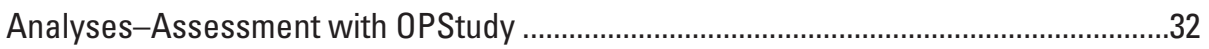

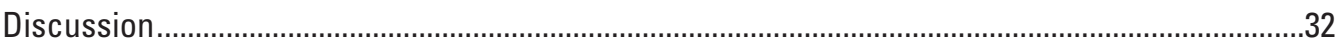

What Have We Learned About the Habitat Model? ...............................................................

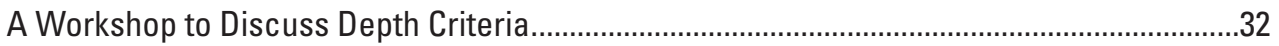

Analysis of Wetted Widths versus Discharge ....................................................................33

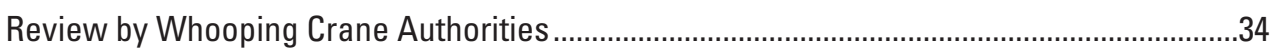

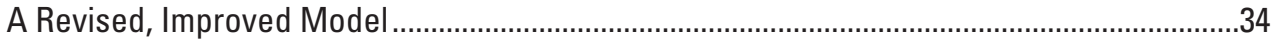

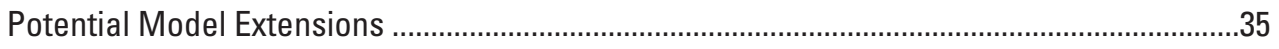

Protocols for Collecting Whooping Crane Observation Data..................................................37

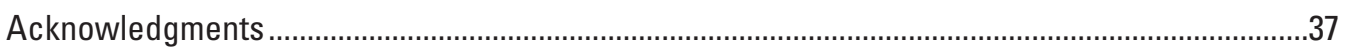

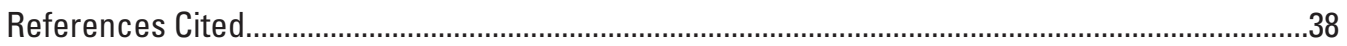

Appendix A. Study Area Maps..............................................................................................

Appendix B. Segment Lengths and Excluded Areas ..................................................................50

Appendix C. Table of UTM Coordinates for Study Site Cross Sections ............................................53

Appendix D. Whooping Crane Roost Observations ...................................................................57

\section{Figures}

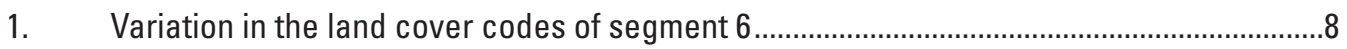

2. The suitability index for wetted width of channel...........................................................10

3. The cumulative frequency distribution of depths of cross sections for 21 separate

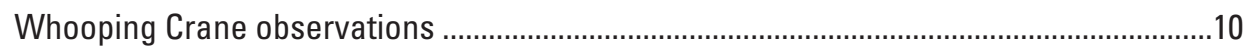

4. A water depth profile constructed for a cross section with two channels........................11

5. Scatter plot and quantiles for average channel width versus number of crane

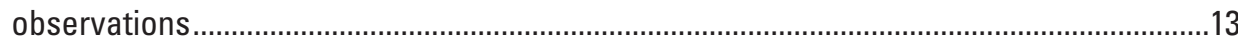

6. Proportionate differences in unobstructed channel width between roost sites used by 46 Whooping Cranes and weighted averages within a segment at a specified discharge

7. Proportionate differences in wetted width of channel between roost sites used by 46 Whooping Cranes and weighted averages within a segment at a specified discharge 
8. Proportionate differences in suitability index based on cumulative frequency distributions of depth between roost sites used by 46 Whooping Cranes and weighted averages within a segment at a specified discharge

9. Proportionate differences in wetted width of channel $<0.7$ feet in depth between roost sites used by 46 Whooping Cranes and weighted averages within a segment at a specified discharge.

10. Wetted widths of channel $<0.7$ feet in depth at 46 Whooping Crane roost sites and corresponding weighted average of wetted widths $<0.7$ feet depth for channels available within the segment at the specified discharge .....................................................................16

11. Cumulative frequency distributions for depth of Study Site 6 cross sections ...........................17

12. The area of suitable roosting habitat as a function of discharge for three maximum depth criteria

13. Proportion of simulated channel wetted widths less than or equal to the wetted widths used by cranes based on three maximum depth criteria

14. Proportionate differences in wetted channel widths used by cranes and the weighted average wetted widths from simulations for three maximum depth criteria

15. Daily flow (cfs) from 4 March-8 May in the Kearney-Odessa hydrologic reach of the Platte River for 1980-2002

16. Daily flow (cfs) from 4 March-8 May in the Grand Island hydrologic reach of the Platte River for $1980-2002$

17. Cumulative distributions of daily flows (cfs) 4 March-8 May in the Kearney-Odessa and Grand Island hydrologic reaches of the Platte River for 1980-2002

18. Flow (cfs) on days Whooping Cranes were observed by median daily flow 4 March8 May at Kearney-Odessa and Grand Island hydrological reaches on the Platte River 1980-2002

19. Whooping Crane observations (triangles) and daily flow (cfs) records from 4 March8 May in the Grand Island hydrologic reach of the Platte River

20. Whooping Crane observations (triangles) and daily flow (cfs) records from 4 March8 May in the Kearney-Odessa hydrologic reach of the Platte River..

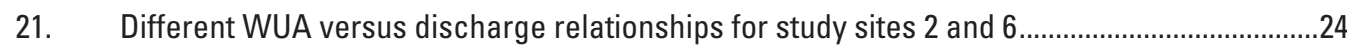

22. The range of WUA (crosshatched band) for discharges in study site 2 …...............................2

23. A flow diagram of the relationships between FORTRAN programs PREFORW, WHOPCWC,

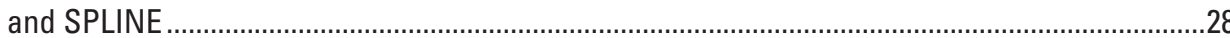

24. The Habitat Suitability Criteria screen in the Platte River Habitat Analysis Program ................30

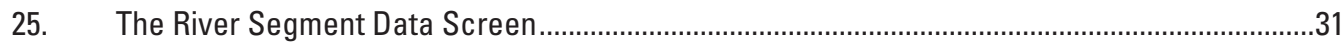

26. The output window produced by a run of the Whooping Crane model with hydrological simulations produced by program OPSTUDY ......................................................................................33

27. Variation in estimated optimal flow as a consequence of different assumptions about the minimum unobstructed width

28. Variation in estimated optimal flow as a consequence of different assumptions about the buffer distance around visual obstructions.

29. Variation in estimated optimal flow as a consequence of different assumptions about the maximum depth constraint

30. Variation in estimated optimal flow as a consequence of different assumptions about the minimum depth constraint. 


\section{Tables}

1. The approximate boundaries of river segments, in river miles, associated with study sites......3

2. Recommended stream gauge and study site associations.....................................................

3. Information about IFG4 data files used in the simulation of discharge versus roosting habitat for Whooping Cranes on the Platte River in Nebraska.................................................... 7

4. Length width, number of roost sightings, and ranks of these variables for river segments represented by specific study sites.

5. A description of 18 alternative depth criteria evaluated for modeling Whooping Crane roosting habitat.

6. Weighted usable area statistics for river segment 6 based on resampling $(n=1,000)$ the field data collected during four separate visits to study site 6 .

7. Statistics on optimal discharges estimated form 1,000 simulations with habitat suitability model C4R 


\section{Executive Summary}

The primary objectives of this evaluation were to improve the performance of the Whooping Crane Habitat Suitability model (C4R) used by the U.S. Fish and Wildlife Service (Service) for defining the relationship between river discharge and habitat availability, and to assist the Service in implementing improved model(s) with existing hydraulic files. The C4R habitat model is applied at the scale of individual river cross-sections, but the model outputs are scaledup to larger reaches of the river using a decision support "model" comprised of other data and procedures. Hence, the validity of the habitat model depends at least partially on how its outputs are incorporated into this larger context. For that reason, we also evaluated other procedures including the PHABSIM data files, the FORTRAN computer programs used to implement the model, and other parameters used to simulate the relationship between river flows and the availability of Whooping Crane roosting habitat along more than 100 miles of heterogeneous river channels. An equally important objective of this report was to fully document these related procedures as well as the model and evaluation results so that interested parties could readily understand the technical basis for the Service's recommendations. Key evaluation results were:

1. We tested Whooping Crane habitat model C4R at two different spatial scales: river segments, and individual cross sections. These evaluations provided support for some of the suitability relations in the model and suggested several improvements.

a. Crane roost observations were not distributed among river segments in proportion to segment length, suggesting that Whooping Crane roosts were not randomly distributed along the river. Instead, cranes selected river segments that had higher average unobstructed widths and higher average habitat suitability as defined by model C4R. These results indicate that habitat selection may be occurring at the river segment scale.

b. At the individual cross section scale, cranes selected roost sites that had greater unobstructed width, greater wetted width, and greater suitability indices for cumulative distribution of depths than the mean values for the segment. These relations were not strongly related to discharge but indicate that model C4R provides a reasonable description of roosting habitat at the individual cross section level.

2. The preceding evaluation of the C4R habitat model shows that it has some utility for predicting river channels more likely to be used by cranes. Evaluation of large-scale patterns indicates that cranes tend to use river segments that, on average, have greater unobstructed channel widths, and the small-scale results show that cranes also tend to select the wider, wetted cross sections within a segment. Thus, the model seems to reflect the generally agreed upon concept that channel width, both unobstructed and wetted widths are important habitat variables. These results support the continued maintenance of wide unobstructed channels and perhaps restoration of channels with low suitability, for long-term management of crane habitat.

3. However, our analyses suggest that there is substantial room for improvement in the model's depth function, which in some form is a necessity if one wishes to continue using the model to estimate target flows in the Platte River study area. The depth function was constructed from the water depth profiles of 21 crane roost observations. The manner in which the depth function (and its component depth profiles) is incorporated into model calculations leads to a serious numerical bias-the estimated optimal flow for a river segment (i.e., the flow that maximizes WUA) is the flow that emulates the water depth profile for a single, historical crane observation. That Whooping Crane group, 88A-2, was observed on 6 April 1988 in a relatively narrow channel (507 feet wide) 
during a period of relatively high flow $(2,680 \mathrm{cfs})$. Almost $90 \%$ of that depth profile was deeper than 0.8 feet (the maximum suitable depth identified in our evaluation), and $70 \%$ was deeper than 1.3 feet (the maximum roosting depth observed for Whooping Cranes).

4. That single, deep crane observation drives all model analyses. If the depth profile is removed from the depth function, the optimal flow for the study area as a whole drops to about 2,000 cfs (from C4R's 2,400 cfs optimal flow). If one removes three other depth profiles for the same crane group (88A-2) on 30 May, 1 April, and 4 April 1988, in relatively narrow channels $(172,495$, and 475 feet) at relatively high flows $(2,460$, 2,680, and 2,450 cfs)), the optimal flow drops another $200 \mathrm{cfs}$ to 1,800 cfs. Hence, the Service's recommended flow targets for Whooping Cranes are based on emulating the depth profiles from a single crane group (88A-2) that chose to roost in relatively narrow channels during a period of relatively high discharge. This problem cannot be overcome by incorporating more future crane observations into the model. The inclusion of new data into the depth function will lead to an even higher predicted optimal flow when a depth profile(s) even deeper than for crane $88 \mathrm{~A}-2$ is discovered.

5. In addition to the problems outlined above, the suitability index for depth $(D F)$ was also a weak predictor of crane usage. Cranes demonstrated a more consistent pattern of selection for an alternative, simpler depth criteria based on depth limits (e.g., suitable roosting habitat occurs from 0.1 to 1.0 feet depth). The selection pattern was nonlinearly related to discharge. Several options for incorporating this new depth criterion were evaluated, and all would improve the model's predictive accuracy.

6. Selecting among the alternative depth criteria that we evaluated could not be reliably done on the basis of the available crane data alone. However, results from a workshop held in February 2002, as well as reviews by several Whooping Crane authorities suggest that the proper depth range for roosting is about 0.1 to 0.8 feet deep. There is some uncertainty, however, and the upper depth limit could be as high as 1.3 feet. Incorporating these new depth criteria into the model results in optimal flow estimates of $1,350 \mathrm{cfs}$ (maximum depth of 0.8 feet) to $1,850 \mathrm{cfs}$ (maximum depth of 1.3 feet).

7. Realizing that crane habitat suitability may be causally related to river discharge, but not in ways that have been perceived by model builders, we evaluated the Whooping Crane data to see if there was any evidence that crane sightings are related to discharge in a general sense. We examined the daily flow records for 4 March to 8 May, corresponding to the dates of earliest and latest observations of Whooping Cranes during spring migration, for the Grand Island and Kearney-Odessa hydrologic reaches for the 23 years comprising 1980-2002. Days on which cranes were observed, number of sightings, and numbers of cranes were identified in the flow record. We evaluated whether the abundance of crane observations was related to season-wide total (or mean) flows, as well as to daily flows within years. There were no patterns indicating that cranes were more frequently observed in high flow years or on days with relatively high flows. We also examined time series plots of daily flows between 4 March and 8 May in each hydrologic reach for each year cranes were observed. Flow on days cranes were observed within these time series exhibited no obvious consistent pattern relative to peak, lowest, rising, or declining flows.

8. Procedures for performing impact assessments at the study area scale must be improved in order to realize the full potential benefits of changing individual habitat suitability criteria or adding new variables to the habitat model.

a. Habitat-discharge relationships for each study site were historically derived using a 'spline' procedure that overemphasizes habitat - discharge relationships at measured flows and masks variability in habitat conditions associated with river dynamics. As a 
substitute for the spline approach, we provide guidelines for applying a 'randomization' approach based on a broad band of habitat-discharge relationships.

b. Habitat changes for the study area should be estimated with records from four stream gauges. Use of a single gauge (Grand Island) ignores known variation in hydrology.

c. We modified several FORTRAN programs, used by the Service to implement the habitat model, and to make them user-friendlier. To make these programs accessible to future Service users, we developed a MS Windows program that provides a user interface and fully integrates data entry, analyses and display. Additional enhancements to these computer programs could be implemented in the future to meet requirements of specific analyses.

9. A number of important future enhancements to the database, habitat model, and related procedures were identified during the course of this evaluation. These include modifying the protocols for collecting more Whooping Crane observations, updating hydraulic aspects of the model (e.g., update data at each of the river study sites, incorporate 2-D modeling of the river, revise the equations for splitting flows among segments), incorporating landscape features into the model (e.g., proximity to wet meadows, roost macroforms, improved disturbance buffers), evaluating the effect of regional landscape dynamics on Crane usage of the Platte River, and developing alternative criteria for defining "optimal" flows. 



\title{
Evaluation of Models and Data for Assessing Whooping Crane Habitat in the Central Platte River, Nebraska
}

\author{
By Adrian H. Farmer', Brian S. Cade', James W. Terrell', Jim H. Henriksen'1, and Jeffery T. Runge ${ }^{2}$
}

\section{Introduction}

\section{Historical Overview of Habitat Model Development}

Beginning in the early 1980's, the U.S. Bureau of Reclamation (Reclamation) and the U.S. Fish and Wildlife Service (Service) initiated the Platte River Management Joint Study (PRMJS) with a purpose, in part, of defining the relationship between river discharge and Whooping Crane (Grus americana) habitat availability in the central Platte River of Nebraska. The principal issue was, and still is, determining the instream flows necessary to provide river-channel roosting habitat for Whooping Cranes, listed as endangered under the Endangered Species Act of 1973, when they migrate through the central Platte River basin in the spring and fall. In reality, there are two time scales associated with this issue, and tools have been developed to deal with the issue in the long- and short-term. The long-term focus is on determining the flow regime necessary to sustain (and perhaps restore) suitable channel morphology throughout the central Platte study area. The short-term focus, on the other hand, is on estimating the instream flow needed to provide roosting habitat, given that channel morphology remains in a dynamic equilibrium around its current state. To address the short-term needs, Reclamation and the Service (USBR, 1987) began developing a habitat suitability model that could be coupled to the Physical Habitat Simulation Model (PHABSIM) (Milhous and others, 1984).

Development of a Whooping Crane habitat suitability model proceeded in several discrete steps. In 1986, two workshops, one by the PRMJS Biology Workgroup and a second by the Service, resulted in the development of an initial habitat suitability index model (Ziewitz, 1992). At that time, data were limited to eight Whooping Crane roosting sites on the Platte River, and channel characteristics had been measured for only three of those sites. The habitat model underwent several progressive reviews by crane authorities and modeling staff at the National Ecology Research Center (now called the Fort Collins Science Center) in Ft. Collins, Colorado. Also during this period, a field protocol was developed for measure-

${ }^{1}$ U.S. Geological Survey, Fort Collins Science Center, 2150 Centre Avenue, Bldg. C, Fort Collins, CO 80526

${ }^{2}$ U.S. Fish and Wildlife Service, 203 W. Second Street, Federal Building, 2nd Floor, Grand Island, NE 68801 ment of habitat variables at roost sites in a manner consistent with the Physical Habitat Simulation (PHABSIM) model, allowing the habitat model to be coupled to hydraulic simulations of the river. During 1987-1990, a number of alternative HSI models were developed and evaluated by the PRMJS based on measurements from additional Platte River roost sites as well as other riverine roost locations in the central flyway (Carlson and others, 1990). Further investigations to refine the model's depth criteria were completed by the subcommittee during 1990-1991.

These continuing efforts to refine a roosting-habitat model resulted in model "C4R" (Anonymous, 1992; Carlson, 1994) developed by the Biology Workgroup of the Interagency Platte River Management Joint Study. Wingfield (1993) first used this model to develop recommendations for instream flows on the Platte River. Since that time, the Service has continued to develop predictions of impacts of proposed flow alterations on Whooping Crane habitat in the Platte River of Nebraska based on Wingfield's original model application. The Service has used model results for Endangered Species Act (ESA) Section 7 consultations with the Forest Service and other Federal agencies on their proposed actions that would result in flow depletions in the Platte River. Model C4R results have been used in several water rights hearings in Nebraska, and the model was the primary tool used to determine the Service's Whooping Crane instream flow targets for the central Platte River. Because of the political interest focused on endangered species and instream flow issues, the C4R habitat model is the subject of intense scrutiny.

In a parallel effort to better understand roosting habitat requirements, the Service also began collecting habitat measurements at crane roost sites in the Platte River in 1966. Through 2002 data were collected for more than 71 individual roost observations, but most of these data were collected since the development of model C4R and had not previously been used to evaluate the model. The National Research Council (2004) further underscored the need to test the habitat model, and urged that calibration of the model be improved. Hence, it would be appropriate to ask, "What do the actual crane observations tell us about the validity of the C4R model?"

\section{Purposes of this Report}

In order to answer this question and to incorporate these new data into future model applications, the Service contracted with the USGS, Fort Collins Science Center to evaluate and update model C4R based on the crane observations, especially 
those observations made since model C4R was developed. The objectives of this effort were to improve the performance of the model for estimating instream flows needed to provide roosting habitat and to assist the Service in implementing improved model(s) with existing hydraulic files.

As background material for this work, the Service provided a large volume of material for our review. This included a notebook entitled "Information Pertaining to Platte River Whooping Crane Roost Habitat and Model Development", that contained several published papers and numerous other file reports that collectively represent the documentation of model C4R, associated instream flow data, and computer programs. The material included a 142-page transcript of the February 20, 1997 court testimony of James Jenniges (General Reporting Services, 1997) that was essentially a critique of model C4R, and a Hydrology Appendix (USBR, 1987) describing field sampling methods and hydraulic models used for habitat simulation. The Service also provided computer disks containing PHABSIM data files, computer programs for running the $\mathrm{C} 4 \mathrm{R}$ model and the crane roost site data collected by the Service from 1966 through 2002.

After an initial attempt to assimilate this background material, two things became apparent. First, we realized that an evaluation of the habitat model would require more than just a test of the model with data from crane roosting sites. For instance, the C4R habitat model is applied at the scale of individual river cross sections, but the model outputs are scaled-up to larger reaches of the river. This scaling-up process is conducted using a decision support "model" comprised of other data and procedures, and the perceived validity of the habitat model depends at least partially on how its outputs are incorporated into this larger context. Hence, we believed that our task also required an evaluation of other procedures including the PHABSIM data files, the FORTRAN computer programs used to implement the model, and other parameters used to simulate the relationship between river flows and the availability of Whooping Crane roosting habitat along more than 100 miles of heterogeneous river channels.

Our second realization slowly emerged from our efforts to understand how the habitat model had been applied to make decisions over the past 10 years or more. We collectively had to invest an enormous amount of time and energy to scale this learning curve. Even after we began to understand what had been done, and why it had been done, we found it impossible to precisely replicate how it had been done. There was no one working for the Service that could run the computer programs that originally implemented the habitat model. Moreover, we could not get all the programs to run on available computers and in some cases we had to rewrite the FORTRAN code to make it run successfully. Consequently, we were never completely certain that we were actually evaluating the same computer models and habitat simulations that had been previously used to support decisions on the Platte River.

Hence, we slowly came to realize that it was at least as important to document the habitat model and its associated procedures, as it was to conduct a technical evaluation of these tools. After all, if we could not replicate past analyses or understand the background technical information given our collective skills and experience in habitat modeling, PHABSIM, and statistical analyses, then this would be an even more difficult task for others with less experience, but who have an interest in understanding the Service's recommendations for instream flows in the Platte River.

Consequently, this report has two purposes. The first purpose is to document an interrelated set of data and models that replicate as closely as possible those that we believe have been used to make flow recommendations so that interested parties can more readily understand the technical basis for the Service's flow recommendations of the past as well as the future. The second purpose is to document our evaluation of the C4R habitat model, and to provide the results of our statistical analyses as well as our recommendations for model modifications that we believe more accurately reflects the relationship between river discharge and Whooping Crane habitat availability in the Central Platte River of Nebraska.

\section{Study Area Description}

\section{The Platte River Study Area}

The study area is the central Platte River channel that has been used historically for nighttime roosting by Whooping Cranes that stopover during spring and fall migration. The specific area of study is defined as all river channels from the $\mathrm{J}-2$ return, near river mile 246, east of Lexington, Nebraska downstream to river mile 157 at Chapman, Nebraska. This 89mile zone includes more than 100 miles of identifiable river channels because the main channel is braided in many places. Furthermore, the hydrologic regime of these individual channels varies substantially throughout the study area. In order to model the flow in multiple channels, and to accurately define the relationship between river discharge and Whooping Crane habitat, it was necessary to stratify the study area into representative reaches and to associate each of the reaches with a specific hydrological reference point.

\section{Hydraulic Study Sites and Segments}

An interagency team of biologists and hydrologists from the Service and Reclamation used PHABSIM, the best available river modeling approach at the time (National Research Council, 2004), to simulate the relationships between river discharge and crane habitat variables, and the results of these simulations (IFG4 files) were provided for our model evaluations. To apply PHABSIM to the entire study area, the team took river measurements at selected cross sections of the river channel. The cross sections are grouped within a "study site", selected to be representative of a larger, relatively homogeneous river reach (termed "segment" in this report). The 
interagency team identified 16 river segments having similar channel morphology and hydrologic conditions (USBR, 1989; USGS, 2000) following guidelines presented in Bovee (1982). Using a stratified random sampling approach, study sites were chosen in each of the 16 segments, and between 3 and 9 cross sections were identified in each of the study sites. Approximately 85 cross sections have been identified in the 16 study sites between Lexington and Chapman, Nebraska, and the Bureau of Reclamation collected data at each of these cross sections on more than one occasion from 1984-1989 (see "PHABSIM data files"). The study site and associated segment(s) were generally given the same identifier (e.g., 12A), but there are some exceptions to this (table 1).

\section{Hydrologic Reference Points}

The discharge-habitat relationships derived from the PHABSIM were coupled to a habitat model to compute an index of habitat availability, "weighted usable area" (WUA), for each river segment. These estimates were summed across all segments to estimate the total WUA at a given river discharge, referenced to the Grand Island, Nebraska gauge. The estimates of total WUA were then used to make decisions about the impacts of flow modifications on the amount of roosting habitat in the entire study area (e.g., Carlson; fig. 2).

However, the simple addition of WUA across segments is meaningful only if the study area is a closed system; e.g., the flow at the upstream end of the study area would result in the

Table 1. The approximate boundaries of river segments, in river miles, associated with study sites.

\begin{tabular}{ll}
\hline Study site & Segment boundaries (river mile) \\
\hline 2 & $239-246$ \\
$4 \mathrm{~A}$ & $227-231$ \\
$4 \mathrm{~B}$ & $208-211,224-227$ \\
$5^{\mathrm{a}}$ & $231-239^{\mathrm{a}}, 212-224$ \\
6 & $200-208,211-212$ \\
7 & $200-212$ \\
$8 \mathrm{an}, 8 \mathrm{as}$ & $187-189,192-196$ \\
$8 \mathrm{~b}$ & $189-192$ \\
$8 \mathrm{c}$ & $196-200$ \\
$9 \mathrm{be}, 9 \mathrm{bw}$ & $168-187$ \\
10 & $171-190$ \\
11 & $168-187$ \\
$12 \mathrm{a}$ & $157-159,161-164$, \\
& $166-168$ \\
$12 \mathrm{~b}$ & $159-161,164-166$ \\
\hline
\end{tabular}

\footnotetext{
${ }^{\mathrm{a}}$ There is no study site in segment 3 ; habitat conditions in segment 3 (river miles 231-239) are predicted by using study site 5 (located in segment 5).
}

same flow at all downstream study sites. Such is not the case for two reasons. First, some of the segments represent individual channels that do not carry the entire river flow at that point. Segments 6 and 7 divide the total flow around Kilgore Island, for example (see maps in Appendix A). The Service and Reclamation developed a series of regression equations based on historic flow data that describe the partitioning of the total flow (TotalQ) within segments with multiple channels $(\mathrm{Q}$ and TotalQ in cfs):

$$
\begin{aligned}
& \text { Kilgore Island (river miles 200-212) } \\
& Q \text { in Segment } 6=\text { Total } Q * 0.68 \\
& Q \text { in Segment } 7=\text { Total } Q * 0.32 \\
& \text { Unnamed Island (river miles 191.5-196) } \\
& Q \text { in Segment 8AS }=\text { TotalQ } * 0.6 \\
& Q \text { in Segment } 8 \mathrm{AN}=\text { Total } Q * 0.4 \\
& \text { Shoemaker Island (river miles 168-187) } \\
& \mathrm{Q} \text { in Segment 9BE }=(\text { TotalQ }-354.48) / 1.81 \text { (aver- } \\
& \text { aged with 9BW to compute WUA) } \\
& \mathrm{Q} \text { in Segment 9BW = Q in Segment 9BE (averaged } \\
& \text { with 9BE to compute WUA) } \\
& \mathrm{Q} \text { in Segment } 10=(\text { TotalQ }-266.48) / 7.35 \\
& Q \text { in Segment } 11=(\text { Total } Q+144.52) / 2.64
\end{aligned}
$$

Second, after taking into account the partitioning of flows among braided channels, there is still considerable variability in monthly mean flows between the gauges throughout the study area. These variations are caused by the routing of water (withdrawal and return flows) and interchange with groundwater within the study area as a whole. For example, during the month of April for a 25-year period (1970-1994), Overton discharges range from $343 \mathrm{cfs}$ more, to $852 \mathrm{cfs}$ less than the discharges at Grand Island and differ from the Grand Island discharge by $200 \mathrm{cfs}$ or more in 11 out of 25 years. For the same time period, Odessa discharges range from $476 \mathrm{cfs}$ more, to $790 \mathrm{cfs}$ less than Grand Island discharges and they differ by $200 \mathrm{cfs}$ or more in 18 out of 25 years. Consequently, assuming a homogeneous discharge throughout the study area as has been done in past applications of model C4R can result in inaccurate estimates of total WUA.

Estimates of total WUA therefore should be determined by adjusting for systematic flow differences between "reaches", which are groupings of segments associated with a specific gauge. The hydrology of the study area is such that the river segments are associated with one of four different gauges: the J-2 return, Overton, Kearney-Odessa, and Grand Island (table 2). The J-2 Return gauge measures the discharge in segment 2; the Overton gauge is associated with segment 3 ; the Kearney-Odessa gauge with segments 4A, 4B, 5, 6 , and 7; and the Grand Island gauge with segments 8AN, 8AS, $8 \mathrm{~B}, 8 \mathrm{C}, 9 \mathrm{BE}, 9 \mathrm{BW}, 10,11,12 \mathrm{~A}$, and 12B. Although flow corrections may have to be implemented in slightly different ways for different planning purposes, we developed Windows 
Table 2. Recommended stream gauge and study site associations.

\begin{tabular}{ll}
\hline Study site/segment & Gauge/hydrologic reach \\
\hline 2 & J-2 return \\
$5 / 3^{\mathrm{a}}$ & Overton \\
$4 \mathrm{~A}$ & Odessa \\
$4 \mathrm{~B}$ & \\
5 & \\
6 & \\
7 & \\
$8 \mathrm{AN}$, & Grand Island \\
$8 \mathrm{AS}$ & \\
$8 \mathrm{~B}$ & \\
$8 \mathrm{C}$ & \\
$9 \mathrm{BW}$ & \\
$9 \mathrm{BE}$ & \\
10 & \\
11 & \\
$12 \mathrm{~A}$ & \\
$12 \mathrm{~B}$ & \\
\hline
\end{tabular}

\footnotetext{
${ }^{\mathrm{a}}$ Habitat conditions should be predicted by using study site 5 cross sectional and hydraulic data and Overton Gauge discharges.
}

computer programs for use by the Service (see COMPUTER PROGRAMS) following the specific segment-gauge associations in table 2.

\section{Study Area Maps}

We were initially unable to develop a clear understanding of the study area stratification described in the preceding sections because there were no detailed maps showing the location of study sites, the boundaries of river segments, and the location of river areas that were excluded as potential Whooping Crane habitat due to human disturbance. Furthermore, the existing documentation was contradictory. For example, Appendix II of Wingfield (1993) indicated that $1 / 2$ mile (or more) on either side of bridges was excluded, whereas Wingfield (1993) and Carlson and others (1990) stated that $1 / 4$ mile on either side of bridges and power lines was excluded.

Because of the problems in study area documentation, we developed our own Geographical Information System (GIS) database of the study area using data themes from numerous sources. Polygonal data were developed from U.S. Bureau of Reclamation archival data themes, surface cover types described by Williamson (1983), cross-section information from spread sheets provided by Duane Woodward of the Central Platte Natural Resources District, and by digitizing boundaries based on descriptions of critical habitat in CFR, Title 50, Part 17.95. Segment boundaries were developed by visually approximating the boundaries shown in fig. 3-1 of USBR (1987). In order to standardize the exclusion of human disturbance areas from our calculations of available habitat, we mapped the areas described in Wingfield (1993) but adjusted them to reflect recommendations of Service biologist Dave Carlson (oral commun., 1999). The large-scale maps developed with our GIS database (USGS, 2000) show the portions of river channel represented by each study site and the location of the segment boundaries, as we believe they were originally intended in USBR (1987).

A map-related problem that continued to arise during our work was the different spatial definitions of "segments" that have evolved in Platte River studies during the last 15 years. Originally, a river segment was defined as a contiguous portion of the channel with the upstream and downstream boundaries fixed by referring to the distance from an easy to locate object such as a bridge or point of an island, as shown in fig. 3-1 of USBR (1987). These original segments are shown on the accompanying maps (Appendix A). As the Service began to develop habitat models, access problems prevented the placement of study sites in all of the original segments, and some segments were subdivided or redefined to better associate homogeneous portions of river with individual study sites. These revised segments were not always continuous reaches of the river, their associated study site(s) was not always within the segment, and the segment boundaries may differ from their original definitions. With the exception of the accompanying maps (Appendix A) showing the original segments as defined by USBR (1987), we have used the newer definitions. The upstream and downstream boundaries of these currently recognized river segments are listed in table 1 and their locations are shown on the accompanying maps as green polygons (Appendix A).

The data themes developed for the accompanying maps provided sufficient information to recalculate the weighted usable area (WUA) associated with each study site using the average reach lengths provided in Appendix B. In a braided channel such as the Platte River, determining "average length" of a selected reach was difficult. Typical approaches used in a single channel, such as following the channel center line, seemed inappropriate because of the numerous crossovers of multiple channels. We calculated "average length" as the mean of the length of the north bank and the length of the south bank of the selected channel (either the main channel or a distinct secondary channel). Bank length was measured along the edge of the water, ignoring small land intrusions whose length exceeded the width of their base. Various sources of human disturbance (e.g., bridges) also affect WUA by excluding areas of the channel that might otherwise be usable by cranes. The maps show the location of human disturbance and the potentially disturbed areas around them. We calculated WUA by excluding these areas of human disturbance from the analyses. Although their intent was to do so, power lines were apparently not excluded from earlier analyses by the Service (e.g., fig. 2 of Carlson, 1994), and to be consistent we also did not exclude these areas when calculating the lengths of channel represented by each study site (Appendix B). 


\section{PHABSIM Data Files}

\section{Collecting and Processing PHABSIM Data Files}

The Service and Reclamation collected channel configuration and hydraulic data at cross sections within study sites using procedures described by U.S. Bureau of Reclamation (1989). The data collected along each cross section consisted of: (1) channel bed and water surface elevations, (2) water depth and velocity data, and (3) land cover codes. Samples of channel bed sediment material also were collected at each study site. Data from each visit to a study site were separately processed using a combination of channel conveyance and step-backwater techniques to produce a separate IFG4 format data file. These IFG4 data files contain the field measurements of bed and water surface elevations, the land cover codes (used for classifying obstructions), and predicted water surface elevations at each cross section for a range of simulated flows. Flow simulations were extrapolated from $40 \%$ to $250 \%$ of the measured flow ( $40 \%$ of the minimum to $250 \%$ of the maximum, if more than 1 measured flow) following PHABSIM guidelines (Bovee, 1982). We accepted these simulation results as supplied and did not rerun the PHABSIM simulations.

The water-surface elevations and channel bed elevations taken from the IFG4 files were then incorporated by other application programs (see Computer Programs) to compute discharge related habitat variables such as "wetted width." The land cover information collected along each cross section plays an important role because it is used to define "visual obstructions", which are used to delimit separate channels and measure "unobstructed channel width", another habitat model variable.

\section{Land Cover Codes Used Before 1998}

The IFG4 file format makes allowances for a special code at each "station" (measurement point) along a cross section. Typically these codes have been used to define substrate type, which is important in habitat models for aquatic organisms. In the Whooping Crane application, this code was used to record information related to visual obstructions. The initial coding system that was begun in the 1980's assigned vegetation codes between 50 and 71 :

1. 50 - a visual obstruction for cranes occurs at the station

2. Other values indicate the distance to the nearest visual obstruction, in 25 foot intervals. For example:

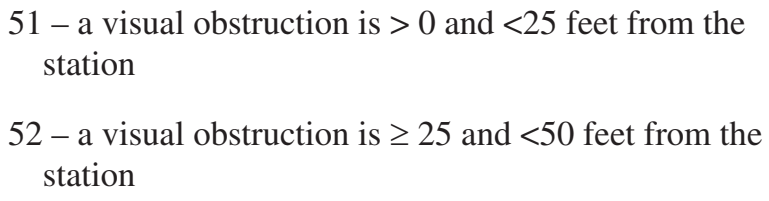
station

52 - a visual obstruction is $\geq 25$ and $<50$ feet from the station

71 - a visual obstruction is $>525$ feet from the station
These codes could potentially be used to adjust the suitability of a station as a function of its distance to an obstruction, but the C4R model did not do this; it uses only the code 50 values to delimit the boundary of channels for estimation of unobstructed channel width.

\section{Land Cover Codes for the 1998-2004 Cross Sections}

A new land cover coding system was begun in 1998 by the Service (Dave Carlson, oral commun., 2002). Existing land cover was assigned a land cover code at points along each cross section. The land cover code consists of three digits in the format "LV.M." Each digit represents a separate land cover feature. The three features are: Land Cover Class, Vegetation Structure, and Vegetation Management.

\section{Land Cover Class}

Refers to the dominant land cover at a station on a cross section. Land Cover Class represents the first digit in the three-digit land cover code. The following values can be assigned to Land Cover Class:

0 barren substrate or annual herbaceous vegetation 4 perennial herbaceous vegetation

5 woody vegetation

6 high bank greater than $1 \mathrm{~m}$ above active channel bed

\section{Vegetation Height}

Refers to the average height of dominant vegetation. Vegetation Height represents the second digit in the three-digit land cover code. The following values can be assigned to Vegetation Height:

0 bare substrate or vegetation $<1 \mathrm{~m}$

1 vegetation $1-5 \mathrm{~m}$ in height

2 vegetation $5-10 \mathrm{~m}$ in height

3 vegetation $>10 \mathrm{~m}$ in height

\section{Vegetation Management}

Refers to any management activity (mowing, disking) that has occurred at the point (station) resulting in reducing the vegetation height to $<1 \mathrm{~m}$. Vegetation Management represents the third digit (decimal position) in the three-digit land cover code. The following are possible Vegetation Management values:

0 not recently managed

1 recently managed 
Upon examining this coding system, we found an apparent inconsistency with established management priorities for the area. With this newer coding system, any point along the cross section that has a land cover code $(=41.0$, or $\geq 50.1)$ is considered a visual obstruction. This system codes all recently managed woody vegetation areas (50.1) as an obstruction, even if there is no vegetation high enough to be an obstruction during the site visit to collect data. This result seems inconsistent with established management priorities within the study area. Some management actions such as burning may have short-lived effects, especially if they are not repeated periodically. But other management actions such as disking can have longer-term effects on vegetation. Furthermore, there are programs (managed by the Service, The Audubon Society, and the Platte River Whooping Crane Management Trust) to periodically alter the vegetation at selected sites. However, the positive results of such management for Whooping Cranes would not be reflected as an improvement if the habitat model and the new coding system were applied to the managed area. This apparent inconsistency may cause problems for the Service in the future, and thus we believe that more flexible coding rules should be considered.

\section{Completeness, Adequacy, and Accuracy of the IFG4 Data Files}

Cross sections at each of the 16 study sites were initially measured between 2 and 9 times at varying river discharges during 1984-1989. This effort resulted in 54 data files in IFG4 format that were prepared by the Platte River Management Joint Study by the USBR (U.S. Bureau of Reclamation, 1987; table 3), and provided to us for this evaluation. Eight of the study sites (Sites 2, 4a, 6, 8b, 8c, 9bw, 9be, and 12a) were resurveyed by the Bureau of Reclamation, Technical Service Center, Denver, in 1998 and 2001, and these 8 additional IFG4 files also were provided for our use.

Each of these 62 hydraulic data files contains both field data and hydraulic model outputs derived from the field data for a single visit to a particular study site. Only part of the data contained in these files is useful for modeling Whooping Crane habitat (see Computer Programs) including: (1) stream bed elevations at the cross sections, (2) discharge measured during the field visit, and (3) predicted water surface elevations (from program Water Surface Profile (WSP)) for a range of model simulations. We crosschecked these data, compared them to published reports (Carlson and others, 1990), and identified two issues that require further resolution.

1. The individual entries in table 3 , extracted from the initial 54 IFG4 data files, did not agree in many cases with a similar table (table A-1) in Carlson and others (1990). In particular, the entries for date, measured discharge, and range of simulation were frequently in disagreement. The individual discrepancies were generally small (e.g., 5-10 cfs), although some were much larger (e.g., $350 \mathrm{cfs}$ ). The entries in our table 3 also differed from the published simulation results developed by the Platte River Management Joint Study (U.S. Bureau of Reclamation, 1987). Since the 1987 USBR report contained actual computer printouts it seemed unlikely that the inconsistencies were due to typographical errors in the published reports. We had no apparent means to resolve these differences with the background information that we were given. However, we later learned (Dave Carlson, oral commun., 1999) that the data files we had been given were revised versions of the original data files and were developed after the Service produced habitat-discharge curves (Carlson and others, 1990) on which its management recommendations have been based. Because the Service's management recommendations were derived from original data files that we could not obtain, we were unable to precisely replicate their earlier modeling results.

2. There appeared to be errors in the vegetation/habitat codes collected at some study sites before 1989 . The problem concerns temporal variation in habitat code '50', the code that was used prior to 1998 to identify a visual obstruction (e.g., tall vegetation) that would constrain effective channel width from the perspective of a Whooping Crane. Effective channel width is an important variable in the habitat suitability model; consequently changes in the amount or location of code 50 along a cross section can significantly affect the computed habitat suitability at that cross section.

Some cross sections show significant variation, between visits, in the number of stations assigned a code 50 . For example, a plot of habitat codes for study site 6 , cross section 2 (fig. 1) shows a large visual obstruction from stations 400-800 feet during the first three visits, but only between stations 500-600 feet on the fourth visit. This difference translates to a 300 feet difference in channel width, which has a significant effect on habitat suitability.

Of course, some of the variation may represent reality; certainly the river channel and associated vegetation change through time in response to hydrologic events. However, based on our discussions with Dave Carlson we believe that some of the apparent variations in code 50 did not actually occur, but rather reflect errors caused by a data reduction process. Earlier versions of the hydraulic models were dimensioned to handle no more than 100 stations per cross section. Therefore, when the original field data for a cross section contained more than 100 stations, some of the data points were arbitrarily eliminated during automated data processing, and some of the eliminated data points were inadvertently located at the transition to code 50 . Thus, in the above example for study site 6 , the apparent 300 feet difference in channel width may simply reflect the fact that "code 50" data points were eliminated in the 300-foot stretch.

Dave Carlson (oral commun., 1999) reviewed the data files and found 16 cases of cross sections (out of approxi- 
Table 3. Information about IFG4 data files used in the simulation of discharge versus roosting habitat for Whooping Cranes on the Platte River in Nebraska. Discharges are at the specific study site, and are not total river discharge.

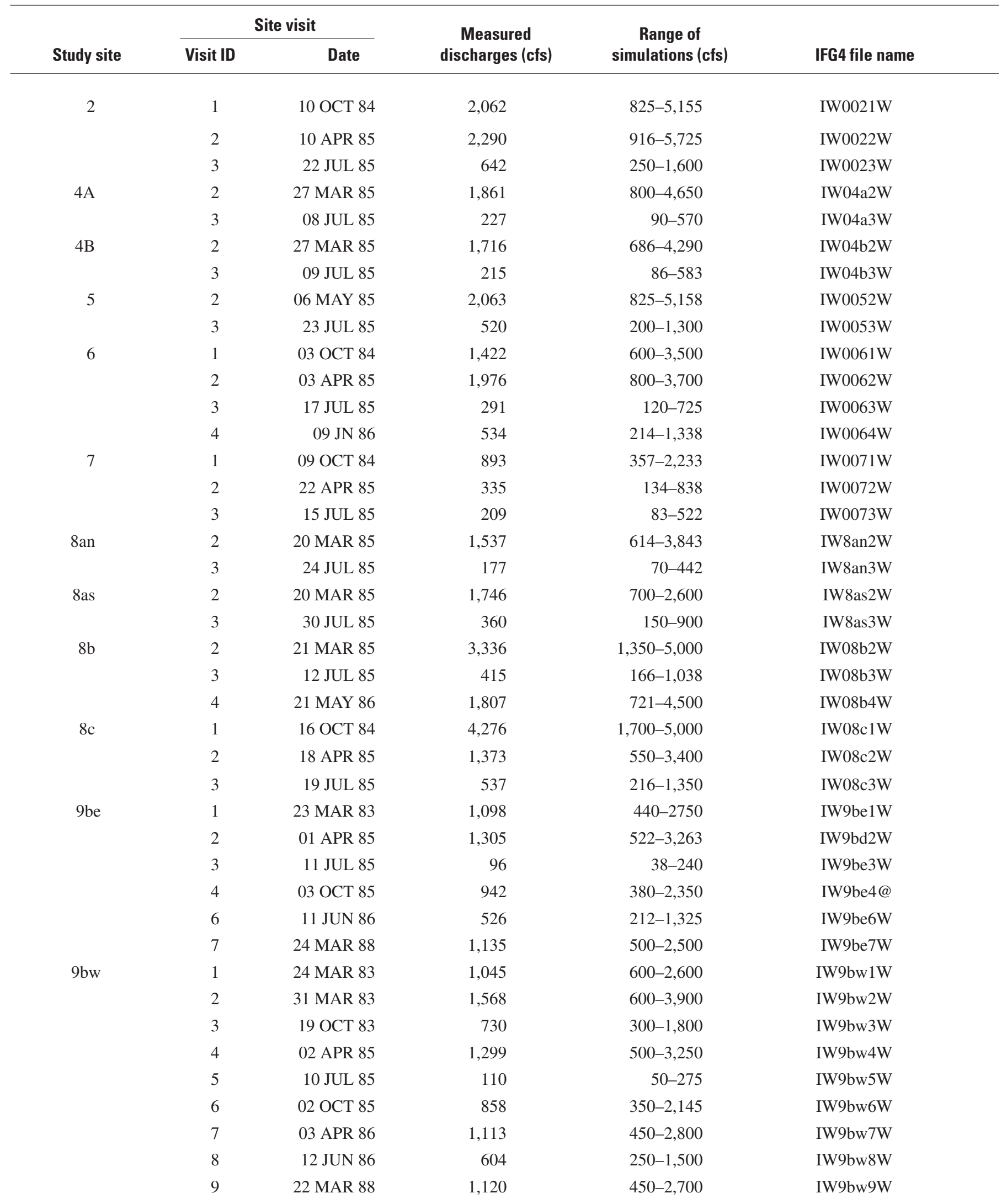


Table 3. Information about IFG4 data files.-Continued.

\begin{tabular}{|c|c|c|c|c|c|}
\hline \multirow[b]{2}{*}{ Study site } & \multicolumn{2}{|c|}{ Site visit } & \multirow{2}{*}{$\begin{array}{c}\text { Measured } \\
\text { discharges (cfs) }\end{array}$} & \multirow{2}{*}{$\begin{array}{c}\text { Range of } \\
\text { simulations (cfs) }\end{array}$} & \multirow[b]{2}{*}{ IFG4 file name } \\
\hline & Visit ID & Date & & & \\
\hline \multirow[t]{4}{*}{10} & 1 & 01 OCT 84 & 464 & $186-1,160$ & IW0101W \\
\hline & 2 & 05 APR 85 & 380 & $152-950$ & IW0102W \\
\hline & 3 & 13 JUL 85 & 34 & $14-240$ & IW0103W \\
\hline & 4 & 23 MAR 88 & 564 & $250-1,400$ & IW0104W \\
\hline \multirow{3}{*}{11} & 1 & 04 OCT 84 & 758 & $300-2,000$ & IW0111W \\
\hline & 2 & 12 APR 85 & 885 & $350-2,200$ & IW0112W \\
\hline & 3 & 20 JUL 85 & 347 & $150-850$ & IW0113W \\
\hline \multirow{4}{*}{$12 \mathrm{a}$} & 1 & 12 OCT 84 & 2,225 & $890-4,500$ & IW12a1W \\
\hline & 2 & 15 APR 85 & 1,837 & $600-3,500$ & IW12a2W \\
\hline & 3 & 16 JUL 85 & 221 & $130-550$ & IW12a3W \\
\hline & 4 & 13 JUL 86 & 1068 & $427-2,670$ & IW12a4W \\
\hline \multirow[t]{2}{*}{$12 b$} & 2 & 16 APR 85 & 1,857 & $743-4,643$ & IW12b2W \\
\hline & 3 & 25 JUL 85 & 598 & $239-1,495$ & IW12b3W \\
\hline
\end{tabular}

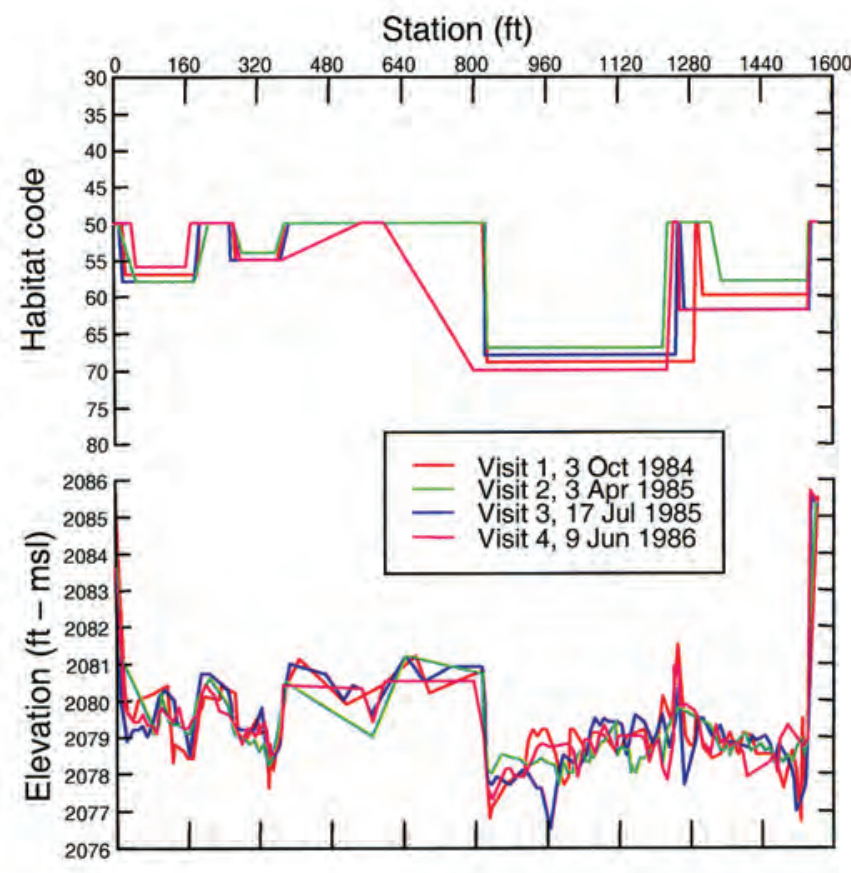

Figure 1. Variation in the land cover codes of segment 6 , cross section 2 apparently due to weeding out data points in the IFG4 data file.

mately 300) that may contain this type of coding error. We have no way of knowing how much error is introduced into the overall analysis due to these coding errors. Certainly each of these coding errors affects the habitat-discharge relationship for that specific cross section, but the degree of bias is diluted when all cross sections within the study site are combined, and diluted even further when all segments are combined to produce a habitat-discharge relationship for the entire study area. Hence, the degree of bias introduced by these inconsistencies is probably small, but the Service should try to resolve these errors, although it may not be possible to recover the original field data and reproduce the IFG4 files at this late date.

\section{Habitat Suitability Model}

\section{Habitat Model Development History}

The Biology Ad Hoc Workgroup sponsored a workshop for the Platte River Management Joint Study in May 1986 with the purpose of developing a habitat suitability model that could be applied to Whooping Crane habitat assessment studies in the Platte River (Shenk and Armbruster, 1986). The workshop identified roosting and feeding requirements of the Whooping Crane on the Platte River for both riverine and nonriverine roost sites. The workgroup identified six criteria that would characterize riverine Whooping Crane roost suitability:

1. Horizontal visibility: The distance from the roost site to the nearest visual obstruction. A visual obstruction is defined as any feature $>1.4 \mathrm{~m}$ in height. Horizontal visibility can be defined spatially as a radius of obstruction free visibility from the roost site. For riverine sites, horizontal visibility is defined as the bank-to-bank distance or unobstructed width of a channel.

2. Disturbance: Human activities or other non-habitat factors that might interfere with crane use of otherwise suitable habitat. 
3. Water depth: The depth of water at potential roost sites is important to providing security to the standing birds.

4. Distance to feeding sites: Location of potential feeding sites in close proximity to potential roosting sites would improve the suitability of a roost site location.

5. Water width: The workshop participants identified two definitions for water width. The first definition is the distance from water's edge to water's edge. A second definition is the unobstructed width of a wetted channel minus beach or sandbars.

6. Water velocity: Workshop participants identified water velocity as an important factor in roost suitability, but the authors stated that there is no current information relating velocity to roost site suitability.

The Service's Nebraska Field Office organized a second workshop in November 1986 to further develop the suitability model. The workshop participants identified three of the six previously identified criteria as being important indicators of riverine Whooping Crane roost suitability (U.S. Fish and Wildlife Service, 1987), and they defined specific measurement protocols:

1. Unobstructed width (a measure of horizontal visibility) was defined as the distance across a channel between visual obstructions. Visual obstructions are defined as either a bank and/or vegetation whose combined height is greater than $1 \mathrm{~m}$.

2. Water width is the summation of all wetted widths within the unobstructed width.

3. Percent water width shallow was defined as the percentage of water width less than or equal to 8 inches in depth.

Although workgroup members supported the concept of suitable roost site velocities, the absence of velocity related information resulted in velocity being removed as a suitability criterion. Of the three criteria selected by workshop participants, water width and percent of width with shallow water are influenced by discharge. Ziewitz $(1987,1992)$ documented the first use of the Whooping Crane habitat model from the November 1986 workshop using PHABSIM. However, these initial efforts evaluated habitat in only 8 of the 16 PHABSIM study sites. The other eight sites were not evaluated because they had an unobstructed width $<500$ feet and the model specified that unobstructed channel widths less than 500 feet were unsuitable.

Several years later, additional changes were made to the November 1986 suitability model (Carlson and others, 1990). These changes resulted from discussions by the Platte River Management Joint Study Biology Workgroup (Carlson and others, 1990). Changes included: (1) unobstructed width $>170$ feet were considered suitable, (2) the wetted width curve was modified, (3) a depth function (DF) based on cumulative frequency distributions (see the next section Description of the C4R Habitat Model) was used in place of percent shallow water width, and (4) disturbance zones were identified. The reduction in minimum unobstructed width to 170 was made because a crane observation on the Loup River showed that cranes used channels as narrow as 172 feet. Disturbance zones were defined to account for reduction in habitat suitability due to the proximity of potential disturbances, defined as any county road, railroad track, bridge, or power line. Power lines, though not a direct disturbance, were included because of their potential as a flight hazard. Any reaches of the river that were within a quarter of a mile of a defined disturbance were supposed to be excluded from calculated area, the equivalent of assigning a suitability rating of 0 . However, areas of disturbance due to power lines were apparently never excluded from area calculations (see page 4 ).

The next refinements to the model resulted in two alternatives for the depth function (Carlson, 1994). The alternatives were labeled C4R (for crane ("C $C$ "), $4^{\text {th }}$ version, range (" $R$ ") and C5R ( $5^{\text {th }}$ version). The differences had to do with how the depth function was actually applied to derive a suitability index (C4R used a proportion and C5R used a binary approach) and the differences were not significant enough to detail here. The C4R approach was eventually selected because it assigned greater WUA to higher and lower discharges (Carlson, 1994).

\section{Description of the C4R Habitat Model}

The C4R habitat model produces an index of habitat suitability for individual channels along a cross section as a function of three habitat variables: unobstructed channel width, wetted width of the channel, and the cumulative distribution of depths within the wetted width. The model assigns a suitability index, from 0.0 (unsuitable) to 1.0 (optimum) (Carlson, 1994), to a river channel based on the following criteria:

a. A channel is assigned a value of 0.0 if the unobstructed channel width is $<170$ feet.

b. For channels that equal or exceed 170 feet in width, the suitability is a product of two indices: a suitability index of the wetted width of the channel (fig. 2), and a suitability index based on cumulative frequency distributions of water depth (fig. 3).

The C4R model is implemented with predicted watersurface elevations from PHABSIM on individual channels within a cross section. The vegetation codes for a cross section are parsed to identify individual channels, delineated by visual obstructions (code 50 prior to 1998 , or code $=41.0$ or $\geq 50.1$ since 1998 as described in Collecting and Processing PHABSIM Data). A cross section may contain two or more separate channels (fig. 4). If the unobstructed width of a channel is $<170$ the suitability is zero; otherwise proceed with the following analyses at each of the simulated discharges:

\footnotetext{
1. A water depth profile is constructed using water surface elevations predicted by PHABSIM combined
} 
with measured channel bed elevations. Wetted width as well as a cumulative frequency distribution of depth is calculated.

2. A suitability index is computed for the wetted-width, and for the cumulative frequency distribution of depth. The index of wetted width is estimated directly from fig. 2. The index for depth is computed as follows. The cumulative frequency distribution is superimposed on fig. 3. If $100 \%$ of the distribution lies within the solid lines (i.e., the envelope of the family of curves in fig. 3 ) then the depth is assigned an index $=1.0$; i.e., an optimal condition. If only $60 \%$ of the distribution lies within the envelope the profile is assigned an index of 0.6 and so on. The suitability index for the channel is computed as a product of the wetted width and depth indices.

3. The suitability index for a channel is multiplied by the surface area of its associated river reach to produce the number of weighted-usable-area (WUA) units associated with the channel. The surface area is defined as the product of the reach length and unobstructed channel width (the distance between the obstructions that delimit the channel).

This 3-step process is repeated for all channels along the cross section and then all cross sections within the study site. The WUA is summed for all channels within a study site, and the sum is converted to another index, the WUA/1,000 feet of channel. This index is multiplied by the length of a river segment (in 1,000's of feet) to compute the WUA in that river segment. However, portions of a river segment are excluded

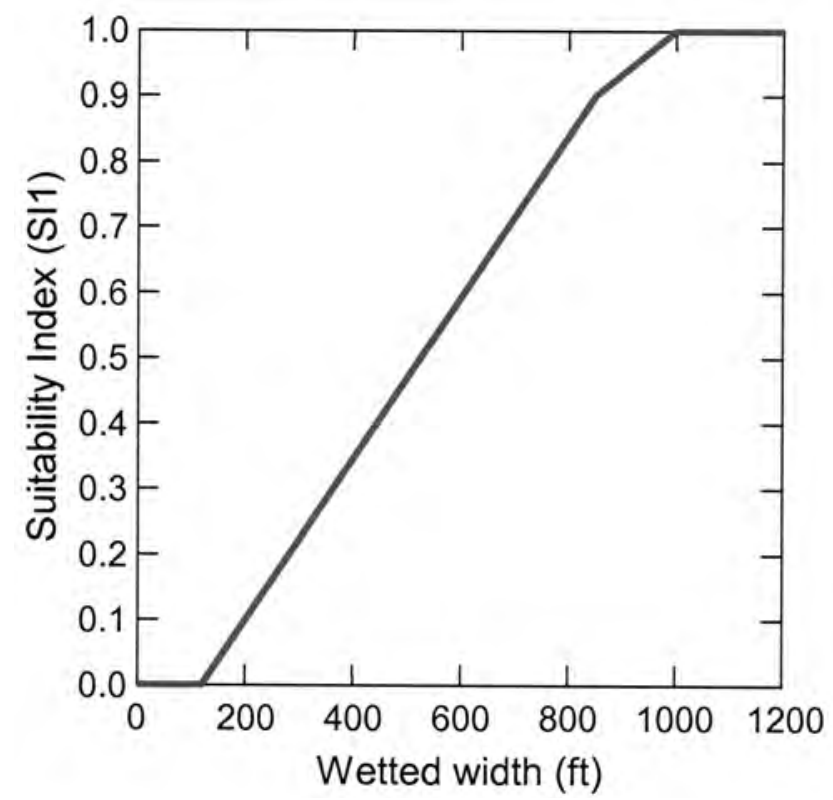

Figure 2. The suitability index for wetted width of channel (from Carlson and others, 1994).

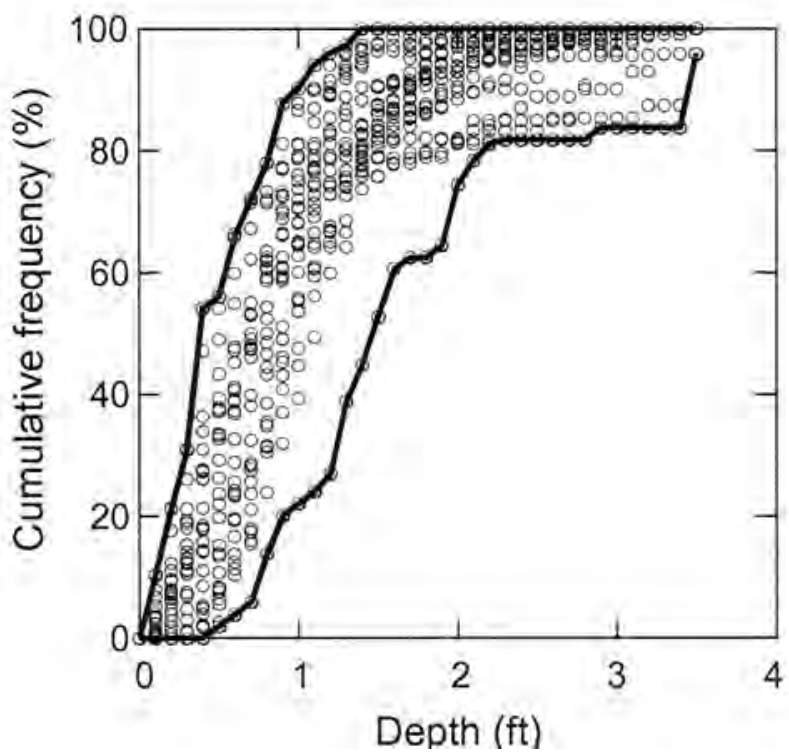

Figure 3. The cumulative frequency distribution of depths of cross sections for 21 separate Whooping Crane observations. The plotted lines are the extreme of the 21 observations, and form an envelope used to compute a suitability index for other river cross sections.

from the length calculation if they are within half mile [although Carlson and others (1990) recommended quarter mile] of a known human disturbance. Hence, disturbances reduce the 'length' of river channel to which the suitability index (WUA/1,000 feet) is extrapolated.

\section{Model Assumptions}

Figure 3 is based on data from 21 Whooping Crane roost observations with depth profiles measured from 1983 through 1989. The approximate river location of these 21 roosts, as well as the river discharge on the observation date was recorded and shortly thereafter a study team returned to the site to measure river depths along a cross section running perpendicular to the flow and through the approximate point where the crane was observed. The depth measurements for each crane sighting were converted to a cumulative frequency distribution, which described the proportion of the cross section that was less than or equal to a given depth. The PRMJS Biology Workgroup subcommittee elected to use this criterion because it was based on actual crane observations. Basing the model on actual crane observations seemed a reasonable decision, but this particular function has been the object of criticism because it is perceived to reduce the suitability of wide, shallow channels (where cranes roost) if there is little or no deep water in the channel (where cranes cannot roost). Furthermore, recent crane sightings (i.e., post-1990) are clustered toward the shallower range (left side of the envelope of fig. 3), suggesting that deeper channels (and higher flows) 


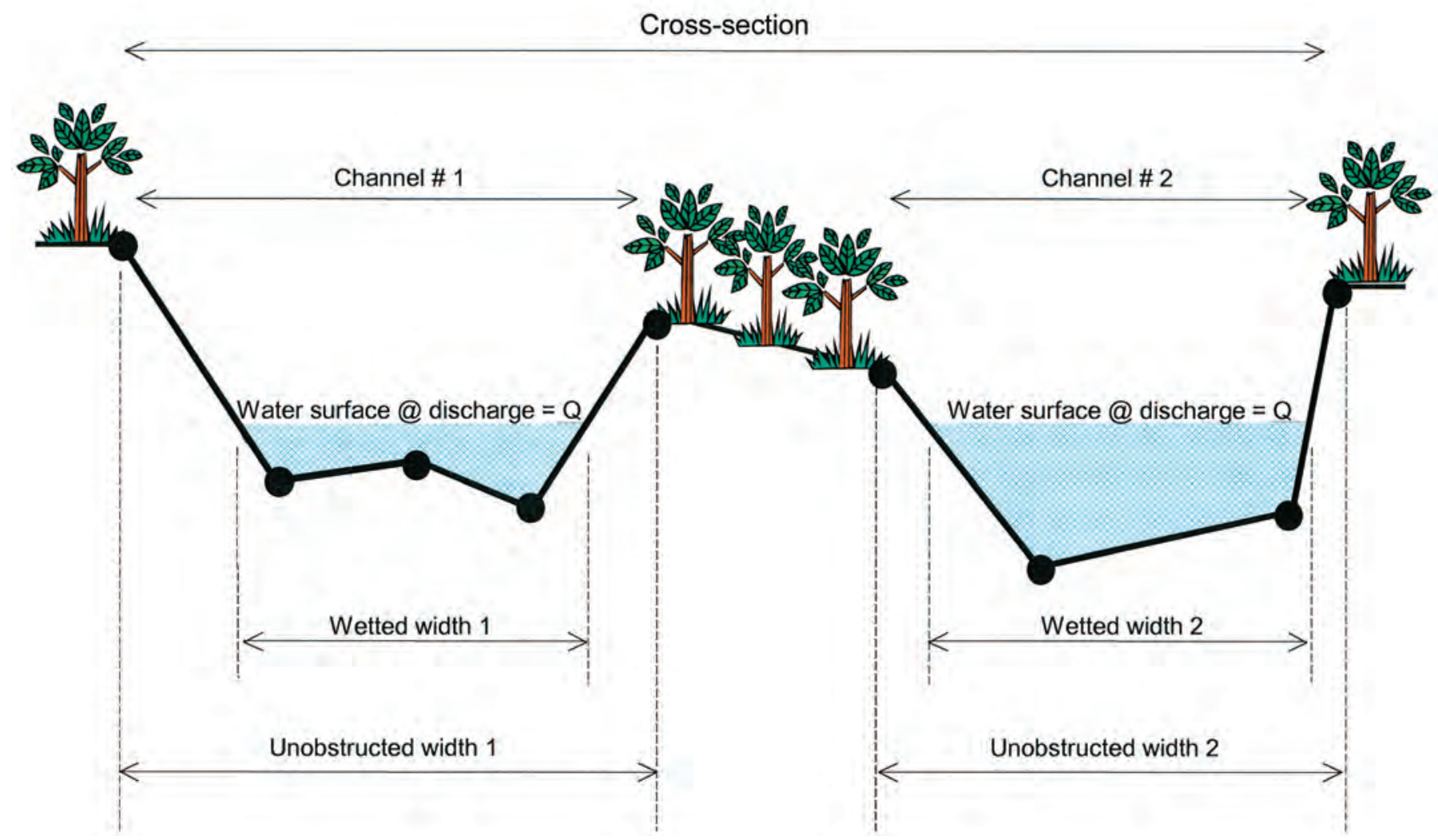

Figure 4. A water depth profile constructed for a cross section with two channels. The unobstructed width of the channels is measured between visual obstructions, which in this case were defined by trees along the banks and on an island. Wetted width is estimated for each simulated discharge (Qi). Filled circles represent the stations where IFG4 data were collected.

would be overvalued by the existing model (we address this issue in more detail in Depth Suitability Criteria).

\section{Habitat Model Evaluation}

The key task that we were asked to perform was an evaluation of the Whooping Crane habitat model in light of the habitat data that have been collected in association with Whooping Crane roost observations on the Platte River in Nebraska. Evaluation of the Whooping Crane model seemed, conceptually at least, to be a straightforward task. We were provided with data on 71 different crane roosting locations, collected from 1966 through 2002 (table D.1). These observations differed somewhat with respect to the available specific data: a depth profile at the site, river discharge at the time, channel widths, and ancillary habitat data from the surrounding river. Our initial idea was to conduct a use-availability analysis, by inputting the habitat data from each roost observation into the C4R model to produce a suitability index for the roost site. These suitability indices could then be compared to the suitability of other 'unused' sites to determine if cranes actually selected the higher suitability sites. If such were the case, then one might begin to trust that the model was realistic. If not, then alternative models would clearly be called for.
In practice, conducting such an analysis was complicated by the fact that there was more than one spatial scale at which the evaluation could be conducted. Whooping Cranes may select a roost site based on the large-scale, landscape characteristics of the site. Such variables as the average width of a segment or segment length might be important cues used by a crane that is flying overhead and looking for a suitable roost site. On the other hand, cranes may select roost sites based solely on the small-scale characteristics of a cross section. Variables such as the wetted width of the cross section or the proportion of the cross section below a certain depth may be important cues for roost selection, independent of the largescale context of the site. Of course, cranes may actually select sites based on both large- and small-scale cues. Thus, we evaluated the model at both spatial scales.

\section{Large-Scale (River Segment) Model Evaluation}

We evaluated the habitat suitability model (C4R) at the level of river segments. We tested two hypotheses concerning the distribution of roost sites with respect to attributes of the 
river channel. First, we were interested to learn if Whooping Crane roost locations were randomly distributed along the Platte River (i.e., there was no apparent selection). If such were the case, then the number of roost locations in each segment would be expected to be proportional to segment length, leading to the following hypothesis:

$\mathrm{H}_{0}$-Whooping Crane (roosting) observations are not proportional to segment length.

$\mathrm{H}_{\mathrm{A}}$-Whooping Crane (roosting) observations are distributed randomly along the river (i.e., the number of observations in each segment is proportional to segment length); versus

\section{Methods}

We tested this hypothesis (in this case, the statistical null hypothesis actually corresponds to a scientific hypothesis of a nonrandom distribution) using two slightly different statistical approaches. First, we evaluated whether there was any significant correlation (Spearman's) between a segment's rank based on length (in $\mathrm{km}$ ) and its rank based on the number of crane observations. Second, we used multiresponse permutation procedures (MRPP) (Mielke and Berry, 2001) to calculate probabilities that river segments containing roost observations were selected randomly from the 15 available segments with respect to their length and rank of length.

We also wanted to determine if Whooping Cranes chose roost sites in wider river channels. There seems to be a consensus among Whooping Crane authorities that channel width is an important determinant of habitat suitability and, indeed, the different versions of the habitat model have included variables pertaining to channel width. We assumed that average width of the study site was representative of an entire segment and tested the following:

$$
\begin{aligned}
& \mathrm{H}_{0}-\text { The number of Whooping Crane (roosting) obser- } \\
& \text { vations is not related to unobstructed channel width; } \\
& \text { versus } \\
& \mathrm{H}_{\mathrm{A}}-\text { Whooping Crane (roosting) observations are } \\
& \text { related to unobstructed channel width. }
\end{aligned}
$$

We tested this hypothesis using the same procedures as for testing segment length. We evaluated whether there was any significant correlation (Spearman's) between a segment's rank based on width and its rank based on the number of crane observations. Second, we used the excess group option of the multiresponse permutation procedures (MRPP) (Mielke and Berry, 2001) to calculate probabilities that river segments containing roost observations were selected randomly from the 15 available segments with respect to their width and rank of width.

Of course, we were also interested in whether roost locations were selected in the more suitable river locations, as defined by the habitat model. We tested the following hypothesis:
$\mathrm{H}_{0}$ - Whooping Crane (roosting) locations are not distributed with respect to segment suitability; versus

$\mathrm{H}_{\mathrm{A}}-$ Whooping Crane (roosting) locations are found in the river segments with the highest habitat suitability, defined as WUA/1,000 feet.

A test of this hypothesis was more problematic. We wished to test for a relationship between the habitat suitability (WUA/1,000 feet) and the number of crane observations per segment. However, this could not be accomplished because a segment's suitability varied with discharge and, moreover, a segment's rank (in terms of habitat quality) also changed with respect to discharge. Thus, we were forced to use individual crane observations as replicates for this test. Each crane observation was assigned the habitat suitability ranking for the segment and the discharge at which it occurred $(1=$ the crane was in the segment with the highest suitability at the discharge; $15=$ the crane was in the segment with the lowest suitability at the discharge). We then did a one-sample permutation test to determine if the ranks of the observations clustered about the median value ( $=8.0$ for 15 segments). If crane observations tended to be centered about the median value, there would be no evidence of selection for quality habitats (as defined by the suitability criteria). Conversely, crane observations displaced from the median toward low values $(<8)$ would be evidence that habitat selection is occurring and would provide support for the habitat model.

\section{Results}

We ranked the river segments with respect to their length [as reported in Wingfield (1993)], width, and number of roost observations, using 59 roost observations that included information on the discharge and channel widths (table 4).

There was no correlation between a segment's rank of length and number of roosting observations $(P=0.487)$. Of 15 segments tested, 10 had roost observations $(04 \mathrm{a}, 04 \mathrm{~b}, 005$, 006, 009, 8an, 8as, 08b, 10, and 12a) and 5 did not (2, 7, 8c, $11,12 \mathrm{~b})$. The probability that the 10 used segments were selected based on length was not significant $(P=0.386)$, and the probability based on rank of length also was not significant $(P=0.384)$. Thus, it appears that Whooping Cranes roost observations are not randomly distributed along the Platte River.

There was a moderate correlation (Spearman's rho $=$ 0.372 ) between a segment's rank of width and number of roosting observations $(P=0.081)$. The probability level of this test declines $(P=0.02)$ if segment $12 \mathrm{~A}$ is excluded. A possible reason to exclude this very wide segment from the analysis is that it is east of the main migration corridor, and as a possible consequence of its location, had only one roost site observation. A scatter plot of the width and roost site data (fig. 5) shows that without this data point (the right-most point near the $\mathrm{x}$-axis at an average width of 1,400 feet) there is an obvious trend of more observations at higher channel widths. Nonlinear functions [number of crane observations $=$ 
Table 4. Length width, number of roost sightings, and ranks of these variables for river segments represented by specific study sites (from Wingfield, 1993).

\begin{tabular}{rrrrccc}
\hline Segment & Length & Rank of length & $\begin{array}{c}\text { Unobstructed } \\
\text { width }\end{array}$ & $\begin{array}{c}\text { Rank of } \\
\text { width }\end{array}$ & $\begin{array}{c}\text { Number of roost } \\
\text { sightings }\end{array}$ & Rank of roosts \\
\hline 2 & 3.9 & 12 & 714 & 5 & 0 & 13 \\
5 & 18.7 & 1 & 579 & 9 & 2 & 6.5 \\
6 & 7.6 & 6 & 936 & 3 & 27 & 1 \\
7 & 11.2 & 4 & 241 & 14 & 0 & 13 \\
9 & 16.3 & 3 & 817 & 4 & 12 & 2 \\
10 & 8.2 & 5 & 202 & 15 & 1 & 9 \\
11 & 16.5 & 2 & 291 & 12 & 0 & 13 \\
4A & 3 & 13.5 & 590 & 8 & 4 & 4 \\
4B & 5 & 10 & 424 & 10 & 3 & 5 \\
8B & 2.3 & 15 & 1,030 & 2 & 7 & 3 \\
$8 \mathrm{C}$ & 3 & 13.5 & 678 & 7 & 0 & 13 \\
12A & 5.4 & 9 & 1,403 & 1 & 1 & 9 \\
12B & 4.4 & 11 & 663 & 6 & 0 & 9.5 \\
$8 \mathrm{AN}$ & 7.3 & 7.5 & 366 & 11 & 0 & 9 \\
$8 \mathrm{AS}$ & 7.3 & 7.5 & 275 & 13 & 1 & \\
\hline
\end{tabular}

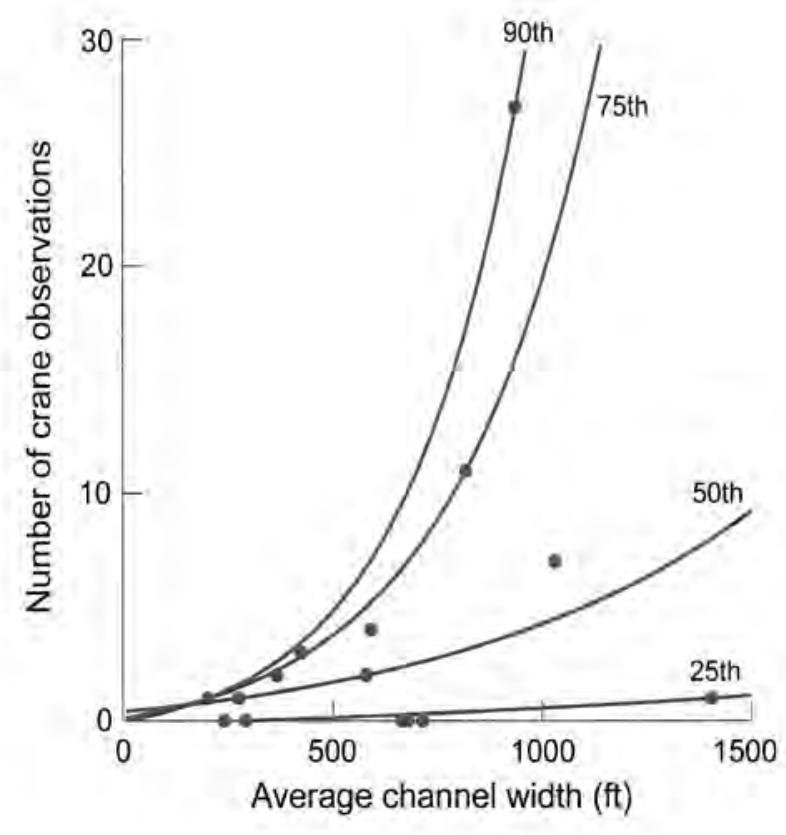

Figure 5. Scatter plot and quantiles for average channel width versus number of crane observations.

$\exp \left(\beta_{0}+\beta_{1}\right.$ average channel width)] for the 15 river segments were estimated with regression quantiles (Cade and others, 1999) for $10^{\text {th }}, 25^{\text {th }}, 50^{\text {th }}, 75^{\text {th }}$, and $90^{\text {th }}$ percentiles. There was a pattern of increasing rates of change with higher quantiles of crane numbers as a function of increasing segment width (fig. 5). An increase in average width of 500 feet increases the $90^{\text {th }}$ quantile of crane numbers by 6 fold whereas the $10^{\text {th }}$ quantile does not increase at all. This pattern is consistent with an interpretation that wide channel width was a necessary condition for large numbers of roosting cranes to occur but was not sufficient to guarantee that cranes would roost in a segment; however, narrow channel widths were sufficient to preclude large numbers of cranes.

We assigned each of the 59 roost observations a ranking (1 to 15 ) based on the segment habitat suitability (WUA/1,000 feet) ranking at the flow measured in the segment at the time of the roost observation. A one-sample permutation test shows a significant displacement from the median rank toward higher suitability ranks $(P<0.0001)$. Thus, it appears that Whooping Crane roosts are located in the higher quality segments as defined by the model C4R. However, it could be argued that some of the earlier observations were actually used to construct the model and, thus, are not independent observations. We repeated the permutation test using only the roost observations made after $1993(n=24)$ and also found a significant relationship between habitat suitability and roost locations $(P<0.001)$.

\section{Small-Scale (Cross Section) Model Evaluation}

We also evaluated model C4R at the level of individual cross sections by comparing the characteristics of cross sections used for roosting by Whooping Cranes with the characteristics of study sites representing the roost locations. 


\section{Methods}

We had 46 observations of habitat use by Whooping Cranes documented by the Service between 1983 and 1998 for which there were recorded discharges and channel depth profiles (table D.1). Each of these 46 channel depth profiles was run through program WHOPCWC (see Computer Programs) at the measured discharge to compute the following variables: (1) unobstructed channel width $\left(U W_{\text {used }}\right) ;(2)$ wetted channel width ( $W W_{\text {used }}$ ); (3) wetted channel width $<0.7$ feet in depth $\left(W W<0.7_{\text {used }}\right)$; and (4) the suitability index for the cumulative frequency distribution of depths $\left(D F_{\text {used }}\right)$.

The habitat considered available for our comparisons was the set of channel cross sections comprising the study site(s) representing the river segment where cranes roosted. Study sites had as many as nine separate cross sections and, at some discharges, some cross sections spanned multiple channels. We used program WHOPCWC to compute the same four variables for each channel at the discharges associated with crane use. We obtained a single estimate of available habitat within a segment at a given discharge by computing a weighted average for each variable $\left(U W_{\text {avail }}, W W_{\text {avail }}, W W<0.7_{\text {avail }}\right.$, and $\left.D F_{\text {avail }}\right)$ by weighting each cross section in a channel by the length of river represented [a default section weighting of 0.5 , meaning the condition on the cross section was extrapolated half the distance to the next cross section, was used following IFIM procedures (Bovee, 1982)].

If Whooping Crane roosts were located randomly with respect to channel characteristics within a segment, the channel variables (1-4 above) at crane roosts would be centered on the measures of available habitat, as defined by the weighted averages defined above. Because our tests were intended to compare channels used and available to cranes across discharges and study sites where available channel characteristics might differ greatly, we chose to standardize variables to proportionate differences [e.g., $\Delta \mathrm{WW}=\left(W W_{\text {used }}-W W_{\text {avail }}\right] /$ $\left.\left.\mathrm{WW}_{\text {avail }}\right]\right)$ before performing statistical tests. We tested the null hypothesis that these proportionate differences $(\Delta U W, \Delta W W$, $\Delta W W<0.7$, and $D F$ ) had a median of zero using a matched pairs variant of multiresponse permutation procedures (Mielke and Berry, 2001).

We were also interested to learn if the proportionate differences changed with discharge. Therefore, we also estimated the change in proportionate differences as a function of river discharge with the $50^{\text {th }}$ (median) regression quantile (Cade and others, 1999). We tested the null hypothesis that rates of change with discharge (i.e., slopes) did not differ from zero with rank-score quantile tests. We also estimated $5^{\text {th }}$ and $95^{\text {th }}$ regression quantiles to describe variation in the functional changes between river discharge for the central $90 \%$ of the proportionate differences.

\section{Results}

Comparisons of crane roosts with available channels indicated that habitat use was not random for all four variables
$(P<0.001)$. Cranes used channels with greater unobstructed width (median $\Delta U W=0.57$, range $=-0.71$ to 1.85 ), greater wetted width (median $\Delta W W=0.58$, range $=-0.71$ to 1.87 ), greater wetted width $<0.7$ feet deep (median $\Delta W W<0.7=$ 1.16 , range $=-0.51$ to 744.15 ), and greater suitability indices for depth based on the depth function (median $\triangle D F=0.12$, range $=0.00$ to 3.54 ). The magnitude of proportionate differences was greatest for $\Delta W W<0.7$ indicating more selective use of roost channels with greater channel widths $<0.7$ feet deep than for the other three variables.

There was little evidence that median proportionate difference in unobstructed channel width (fig. 6) and wetted channel width (fig. 7) changed as a linear function of discharge $(P>0.175)$ based on slopes of $50^{\text {th }}$ regression quantile estimates. Thus, crane habitat selection for these variables appears to be constant across discharge. However, the variability of the proportionate difference in wetted widths (as measured by $\Delta W W$ ) increased with discharge (fig. 7), indicating less consistent selection at higher discharges. Medians for the depth suitability index $(D F)$ increased with discharge (fig. $8 ; P<0.001$ for slope of $50^{\text {th }}$ regression quantile), but the estimated increase in proportionate differences across the $3,000 \mathrm{cfs}$ of observed discharges was only 0.33 . There was a strong, heterogeneous nonlinear increase in selective use of wetted widths of channels $<0.7$ feet deep $(\Delta W W<0.7)$ as a function of discharge (fig. 9; $P=0.069$ for slope of $50^{\text {th }}$ regression quantile), with increases across $3,000 \mathrm{cfs}$ an order of magnitude greater than for $\triangle D F$. Variation in selective use of wetted widths of channels $<0.7$ foot deep increased at higher discharges as indicated by the spread in slopes between $5^{\text {th }}$ and $95^{\text {th }}$ regression quantile estimates (fig. 9), but all rates

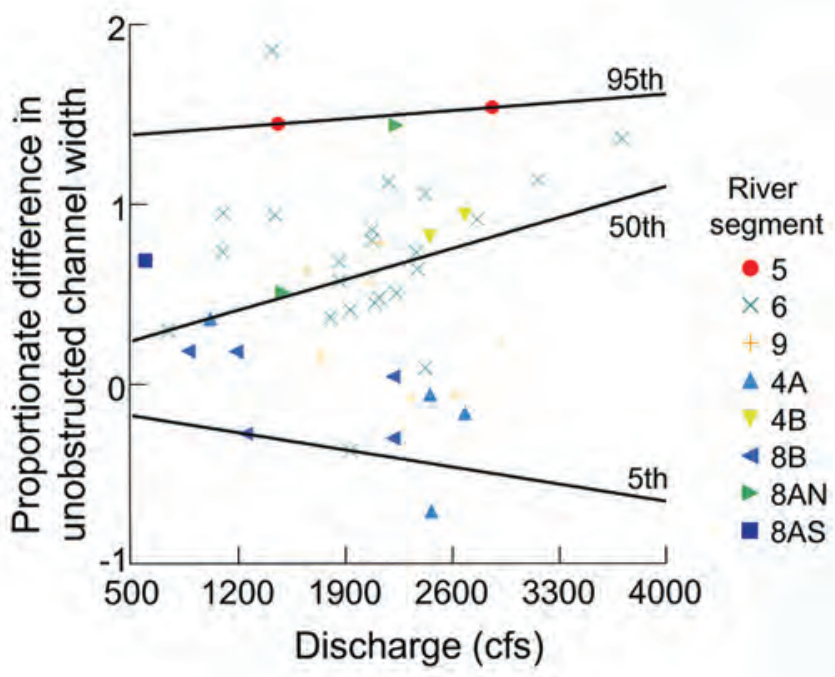

Figure 6. Proportionate differences in unobstructed channel width between roost sites used by 46 Whooping Cranes and weighted averages within a segment at a specified discharge. Functions plotted are the $5^{\text {th }}, 50^{\text {th }}$, and $95^{\text {th }}$ regression quantile estimates for linear model $y=\beta_{0}+\beta_{1} X+\varepsilon$. Slope estimates are -0.0001 for $5^{\text {th }}$, 0.0002 for $50^{\text {th }}$, and 0.00006 for $95^{\text {th }}$ quantiles. 


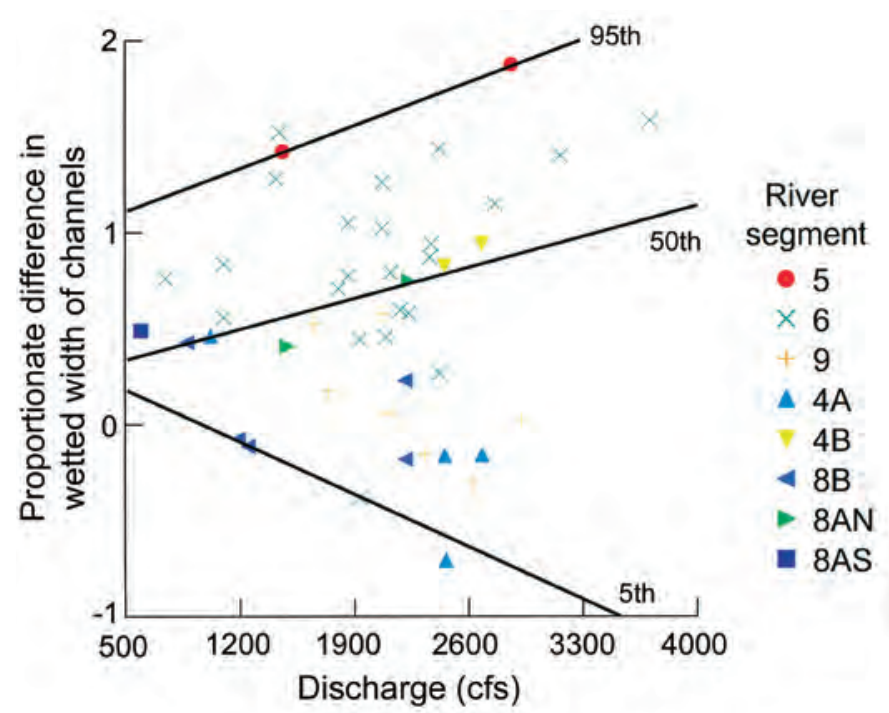

Figure 7. Proportionate differences in wetted width of channel between roost sites used by 46 Whooping Cranes and weighted averages within a segment at a specified discharge. Functions plotted are the $5^{\text {th }}, 50^{\text {th }}$, and $95^{\text {th }}$ regression quantile estimates for linear model $y=\beta_{0}+\beta_{1} X+\varepsilon$. Slope estimates are -0.0004 for $5^{\text {th }}$, 0.0003 for $50^{\text {th }}$, and 0.0003 for $95^{\text {th }}$ quantiles.

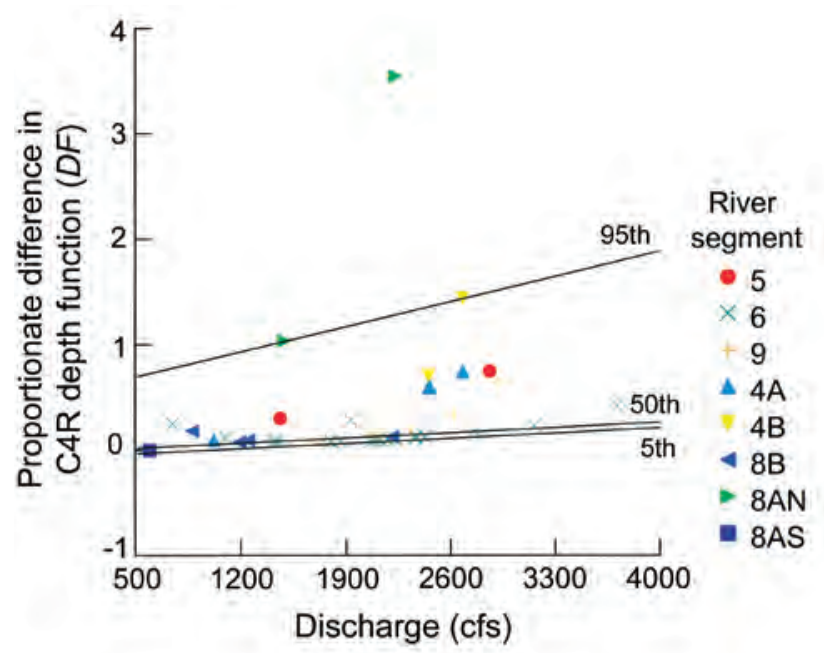

Figure 8. Proportionate differences in suitability index based on cumulative frequency distributions of depth (DF) between roost sites used by 46 Whooping Cranes and weighted averages within a segment at a specified discharge. Functions plotted are the $5^{\text {th }}$, $50^{\text {th }}$, and $95^{\text {th }}$ regression quantile estimates for linear model $y=$ $\beta_{0}+\beta_{1} X+\varepsilon$. Slope estimates are 0.0002 for $5^{\text {th }}, 0.0001$ for $50^{\text {th }}$, and 0.0003 for $95^{\text {th }}$ quantiles.

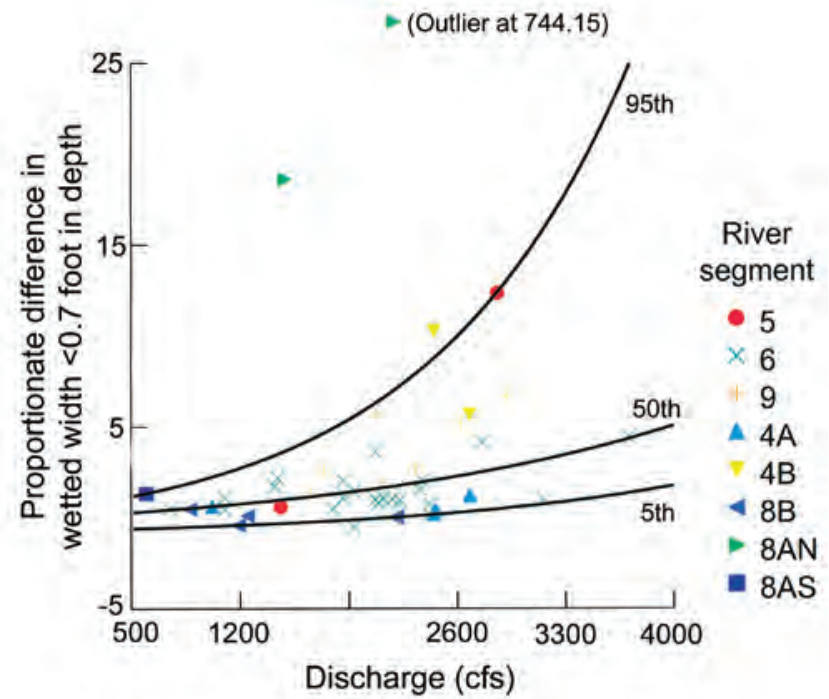

Figure 9. Proportionate differences in wetted width of channel $<0.7$ feet in depth between roost sites used by 46 Whooping Cranes and weighted averages within a segment at a specified discharge. Functions plotted are the $5^{\text {th }}, 50^{\text {th }}$, and $95^{\text {th }}$ regression quantile estimates for linear model $y=\beta_{0}+\beta_{1} X+\varepsilon$. Slope estimates are 0.0006 for $5^{\text {th }}, 0.0008$ for $50^{\text {th }}$, and 0.0011 for $95^{\text {th }}$ quantiles.

of change were positive and skewed towards higher selection at greater discharges. The pattern in $\Delta W W<0.7$ occurs because available wetted widths of channels $<0.7$ feet deep decreased greatly with an increase in discharge, whereas the channels used by cranes had more constant distribution of wetted widths of channels $<0.7$ feet deep across discharges (fig. 10).

\section{Depth Suitability Criteria}

Our preceding evaluation of the $\mathrm{C} 4 \mathrm{R}$ habitat model shows that it has some utility for predicting river channels more likely to be used by cranes. Evaluation of large-scale patterns indicates that cranes tend to use river segments that, on average, have greater unobstructed channel widths, and the small-scale results show that cranes also tend to select the wider, wetted cross sections within a segment. Thus, the model seems to reflect the generally agreed upon concept that channel width, both unobstructed and wetted widths are important habitat variables. These results support the continued maintenance, and perhaps restoration, of wide unobstructed channels in the study area for long-term management of crane habitat. However, our analyses suggest that there is substantial room for improvement in the depth function, which is a necessary component of the model for setting target flows in the Platte River study area.

The suitability index for depth $(D F)$ was a weak predictor of crane usage. The cumulative frequency distributions used to construct the depth function were based on actual river cross sections that contained some deep water not usable by cranes. The cross sections were assumed to be optimal, 


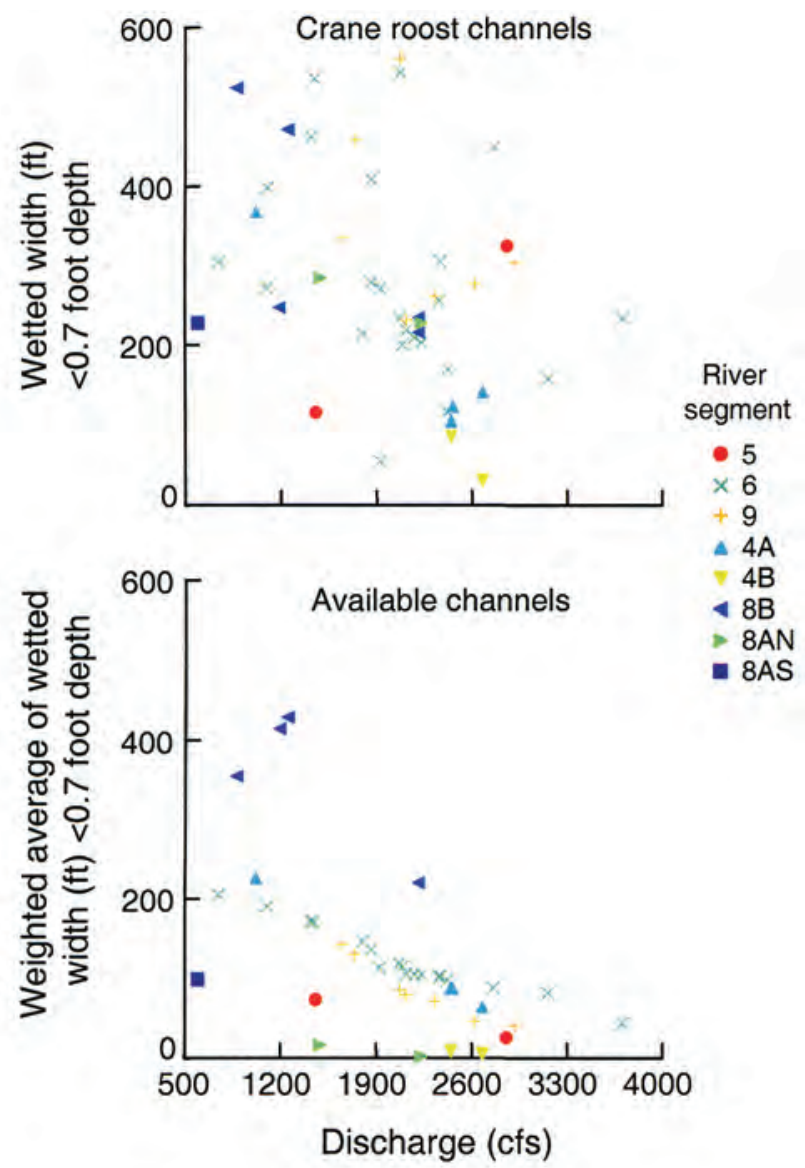

Figure 10. Wetted widths of channel $<0.7$ feet in depth at 46 Whooping Crane roost sites and corresponding weighted average of wetted widths $<0.7$ feet depth for channels available within the segment at the specified discharge.

leading therefore to the implicit assumption that deep water is required along a cross section for optimal conditions. The importance of deep water to Whooping Cranes is not documented in model reports, nor are we aware of any ecological rationale that supports the need for deep water. Faanes and others (1992) found that deeper water surrounded shallow Whooping Crane roost sites more frequently than "unused" sites. We disagree with their statistical analysis, however, because their "unused" sites included deep sites (D. Johnson, written commun., 2004) that by definition are less likely to be surrounded by deeper water, and they failed to account for available depth profiles changing with discharge (i.e., differences between used and "unused" depths were confounded by depth changing with discharge). Nevertheless, if deep water were important for Whooping Cranes as they speculated that it might be, its spatial location with respect to potential, shallow roost sites also would be important. The suitability index $D F$, however, does not take spatial location into account. For the above reasons, the depth function has been the object of past criticism. However, we believe that it has a more serious flaw that apart from the above is ample reason alone to reject this particular function.

A fundamental problem with the depth function is the implicit assumption that all 21 Whooping Cranes represented in fig. 3 were roosting along a cross section with an optimal depth profile. Conceptually, the fact that an animal uses a particular place does not lead to the conclusion that the habitat conditions at that location are optimal. Animals commonly occur in sub-optimal habitats for many reasons including that optimal habitats are not available to them. Moreover, even if the actual point at which a Whooping Crane roosted had optimal depth, it could not be logically inferred that the depths along a cross section of arbitrary length, which passed through the roosting point, were also optimal. The depth function, however, makes such an extrapolation to a larger spatial context without supporting ecological information. Because the 21 arbitrary cross sections intersected some deeper spots, such deep water (up to 3.5 feet deep) has become a requirement for optimality even though such deep spots may be hundreds of feet from the shallow spots on which cranes actually roosted. This concern was highlighted by a previous reviewer who pointed out that if the river cross sections were arbitrarily extended to encompass other landscape features, for example I-80 that runs parallel to the central Platte, similar logic would require one to conclude that an interstate highway is needed in order to have optimal Whooping Crane roosting habitat [court testimony of James Jenniges (General Reporting Services, 1997)].

It seems questionable that any of the 21 observations represent an optimal depth profile, much less all of them, because these observations occurred across such a wide range of channel widths (172-1,365 feet) and discharges (747-3,710 cfs). Instead, fig. 3 simply shows the conditions that 21 cranes selected, from the habitats that were available to them on 21 separate days. To illustrate this, we compared the cumulative frequency distributions for four of the 21 Whooping Cranes seen in segment 6 ("used") with the cumulative frequency distributions from the study site cross sections at the same discharge ("available") (fig. 11). The distribution for each of these crane observations is very typical of the set of available conditions. As discharge increases, the available cross sections become deeper (i.e., they shift to the right on the graph), and the cross section used by cranes changes to reflect the available conditions, which are tightly constrained.

\section{A Mathematical Bias toward High Flows}

In addition to the conceptual issues regarding optimality, a serious numerical bias occurs when using the C4R model to establish flow targets. The cumulative frequency distributions along the right margin of the envelope in fig. 3 are for deeper cross sections. The lower-right boundary of the envelope in fig. 3 represents Whooping Crane 88A-2, which roosted on the river on 6 April 1988 in a 507 foot wide channel with a river discharge of 2,680 cfs (see table D.1). 

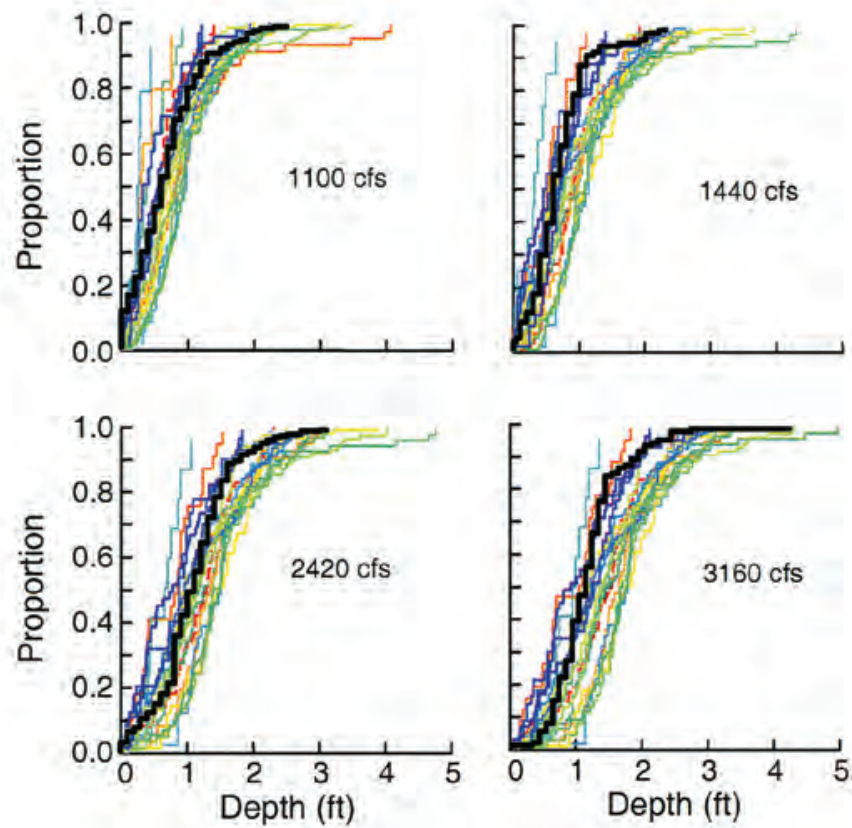

Figure 11. Cumulative frequency distributions for depth of Study Site 6 cross sections: black lines are individual Whooping Crane roost observations; colored lines are study site transects representing the available habitat at the discharge measured when that crane was present in the river.

Almost $90 \%$ of that depth distribution was deeper than 0.8 feet (the maximum suitable depth identified in our evaluation), and $70 \%$ was deeper than 1.3 feet, the maximum roosting depth observed for Whooping Cranes (Austin and Richert, 2001; Austin, oral commun., 2004).

This single crane observation essentially drives all model analyses. When the model is run to define the optimal flow (i.e., the flow that would maximize the amount of roosting habitat), the model attempts to emulate these deep conditions in all river segments. This happens as follows. The habitat model is typically run for a range of discharges (see Description of the C4R Model). As discharge increases, so does wetted channel width. As wetted channel width increases so does suitability, up to 1,000 feet in width. At high discharges however, the channel begins to become too deep and the depth suitability begins to decline. The discharge that maximizes overall suitability (a product of the wetted width and depth indices) is the discharge that produces the widest possible wetted channel width, while also maintaining an "optimal" depth profile.

This solution occurs for a depth profile at the right side of the fig. 3 envelope because this relatively deep condition: (1) has an "optimal" depth, and (2) the widest possible wetted width consistent with (1). The crane roost observations in the middle and along the left margin of the envelope in fig. 3 have no influence on the model results.

We removed the distribution for crane $88 \mathrm{~A}-2$ on 6 April 1988 from the depth function (fig. 3) and re-ran the C4R model. The flow that maximizes WUA drops to about 2000 cfs (95\% confidence interval of 1,700-2,500 cfs) from C4R's $2,400 \mathrm{cfs}$ (95\% confidence interval of 2,000-2,950 cfs) optimal flow. We also removed three other crane observations that collectively form the upper-right boundary of the envelope of fig. 3. These observations were also for the same crane group (88A-2) on 30 May, 1 April, and 4 April 1988, in relatively narrow channels $(172,495$, and 475 feet) at relatively high flows $(2,460,2,680$, and $2,450 \mathrm{cfs})$. When these three additional cross sections were removed the maximizing discharge dropped another $200 \mathrm{cfs}$ to about 1,800 cfs (95\% confidence interval of $1,550-2,150 \mathrm{cfs}$ ).

Hence, the Service's recommended flow targets for Whooping Cranes are largely based on emulating the cross sections from a single crane group (88A-2) that chose to roost in relatively narrow channels during a period of relatively high discharge. Removing just a single night's observation from the depth function decreases the maximizing discharge by $400 \mathrm{cfs}$, and removing the four observations of crane group $88 \mathrm{~A}-2$ decreases the maximizing discharge by $600 \mathrm{cfs}$. This model flaw is not related to a lack of data, and in fact cannot be overcome by incorporating more future crane observations. The inclusion of new data can only lead to a more extreme prediction when a depth profile(s) even deeper than for crane 88A-2 is discovered. For these reasons we strongly recommend that the model's depth function be replaced with a more ecologically justifiable function that can be improved as more data become available.

\section{Alternative Depth Functions}

Alternative approaches for constructing depth functions were discussed at a workshop in Grand Island, Nebraska on 13 and 14 February 2001 (Farmer and others, 2001). Alternatives considered in detail were based on replacing the depth function with a much simpler variable such as: (1) the wetted width of channel $<0.7$ feet deep, or (2) the wetted width of channel $<0.7$ feet deep and more than 80 feet from an obstruction. Such a variable was, in fact, used in an earlier version of the model. The preceding evaluation showed that wetted width $<0.7$ feet has the strongest pattern of selective channel use by cranes as a function of discharge. Furthermore, such a simple depth constraint (though perhaps a value different than 0.7 feet) is easier to justify on ecological grounds, and is easier to test and refine as more data become available in the future.

\section{Comparing Depth Criteria Based on Crane Observations}

There would be several ways to implement a variable based on simple depth constraints, and we evaluated 18 alternatives based on a range of depths (table 5). We made no judgments about the relative suitability of these alternatives for Whooping Cranes, but were more interested in evaluating a different concept than model C4R's depth function. Each 
Table 5. A description of 18 alternative depth criteria evaluated for modeling Whooping Crane roosting habitat. For each criterion, suitable roost sites have water depths between the specified minimum and maximum and must be at least as far as "Buffer" from a visual obstruction.

\begin{tabular}{cccc}
\hline Criterion & $\begin{array}{c}\text { Minimum } \\
\text { depth (feet) }\end{array}$ & $\begin{array}{c}\text { Maximum } \\
\text { depth (feet) }\end{array}$ & Buffer (feet) \\
\hline 1 & 0.0 & 0.7 & 0.0 \\
2 & 0.0 & 1.0 & 0.0 \\
3 & 0.0 & 1.5 & 0.0 \\
4 & 0.0 & 0.7 & 40.0 \\
5 & 0.0 & 1.0 & 40.0 \\
6 & 0.0 & 1.5 & 40.0 \\
7 & 0.0 & 0.7 & 85.0 \\
8 & 0.0 & 1.0 & 85.0 \\
9 & 0.0 & 1.5 & 85.0 \\
10 & 0.25 & 0.7 & 0.0 \\
11 & 0.25 & 1.0 & 0.0 \\
12 & 0.25 & 1.5 & 0.0 \\
13 & 0.25 & 0.7 & 40.0 \\
14 & 0.25 & 1.0 & 40.0 \\
15 & 0.25 & 1.5 & 40.0 \\
16 & 0.25 & 0.7 & 85.0 \\
17 & 0.25 & 1.0 & 85.0 \\
18 & 0.25 & 1.5 & 85.0 \\
\hline
\end{tabular}

of the alternative criteria was applied in a binary fashion: any portion of the river channel between the specified minimum and maximum depths, and at least a "buffer" away from a visual obstruction was deemed to be suitable. All other portions of the river channel not meeting the criteria were deemed unsuitable. In turn, each of the criteria was substituted in the model for the cumulative depth criteria, and a series of computer runs were made with the model to compare the model's depth criteria, and the 18 alternatives based on the crane observations.

The amount of WUA was plotted against discharge for the $<0.7,<1.0$, and $<1.5$ foot depth criteria for segment 6 . There was a strong decline in WUA with discharge for both the $<0.7$ and $<1.0$ foot depth criteria but very little change with the $<1.5$ foot depth criterion both for individual channel wetted widths and for average wetted widths as estimated by a locally weighted smoothing function (fig. 12).

The alternative depth criteria were evaluated with the crane observations (those observations with accompanying depth profiles) from river segment 6 , where the majority of crane roosting observations occurred $(n=23)$. We computed the proportion of the available channels for which wetted width was less than or equal to the wetted width of the channel where the cranes were roosting, and also computed these

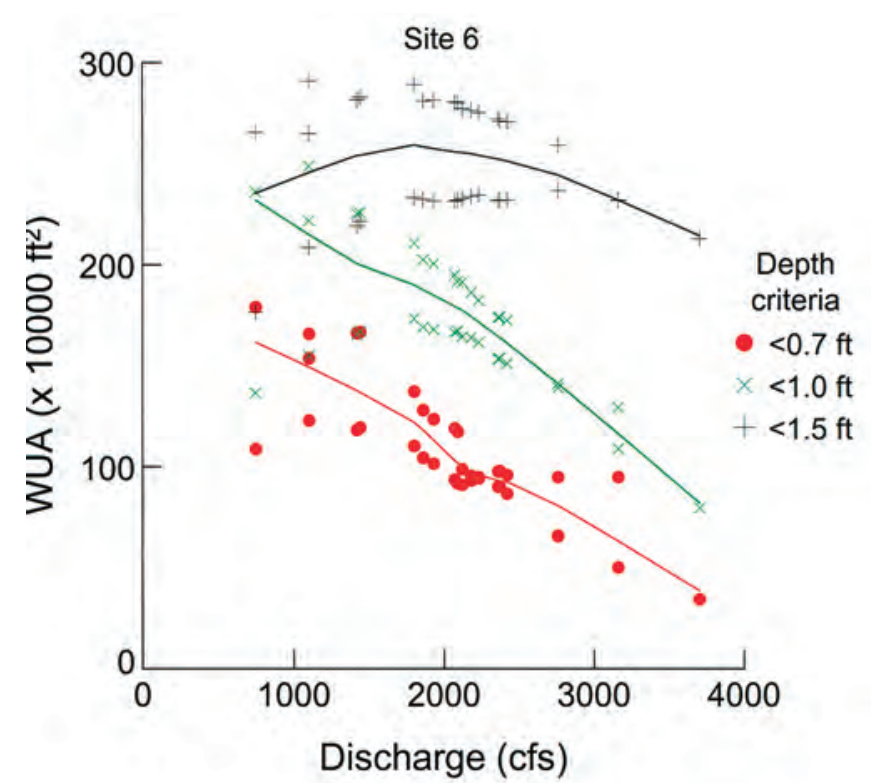

Figure 12. The area of suitable roosting habitat as a function of discharge for three maximum depth criteria (minimum depth $=0$, buffer $=0$ ) in segment 6 .

proportions based on the suitability index for the original depth function $(D F)$. These proportions were then tested against the null hypothesis that they were centered about 0.5 (the median) using a matched pairs variant of multiresponse permutation procedures (Mielke and Berry, 2001). This null hypothesis corresponds to channel widths used by cranes having the same distribution as channel widths available at a given discharge; i.e., cranes are choosing roost sites randomly. We also compared channels used by cranes to those that were available within segment 6 at the discharges corresponding to crane use based on proportionate differences [e.g., $\left.\left.\Delta \mathrm{WW}<0.7=\left(W W<0.7_{\text {used }}-W W<0.7_{\text {avail }}\right] / W W<0.7_{\text {avail }}\right)\right]$ as the summary variable. The null hypothesis that proportionate differences were centered about zero was tested using the matched pair's variant of the multiresponse permutation procedures (Mielke and Berry, 2001). This latter test assumed that channel-width availability in segment 6 was proportional to representative channel widths within study site 6 .

Within segment 6 there was little evidence that channels used by cranes differed from those available when based on the original depth function $(D F)$ (MRPP, $P=0.717$ ). If anything, the analysis suggests that there were many channels $(>50 \%)$ available that were similar to the suitability of channels used by cranes when described by the depth function (all crane transects had a 1.0 suitability), especially at lower discharges (fig. 13). However, the $<0.7,<1.0$, and $<1.5$ foot depth criteria indicated that channels used by cranes were greater in width than $70 \%$ of the channels available at a given discharge (MRPP, all $P<0.0001$ ).

The proportionate differences based on weighted averages for available channels also indicated nonrandom selection for the $<0.7,<1.0$, and $<1.5$ foot depth criteria (MRPP, all 


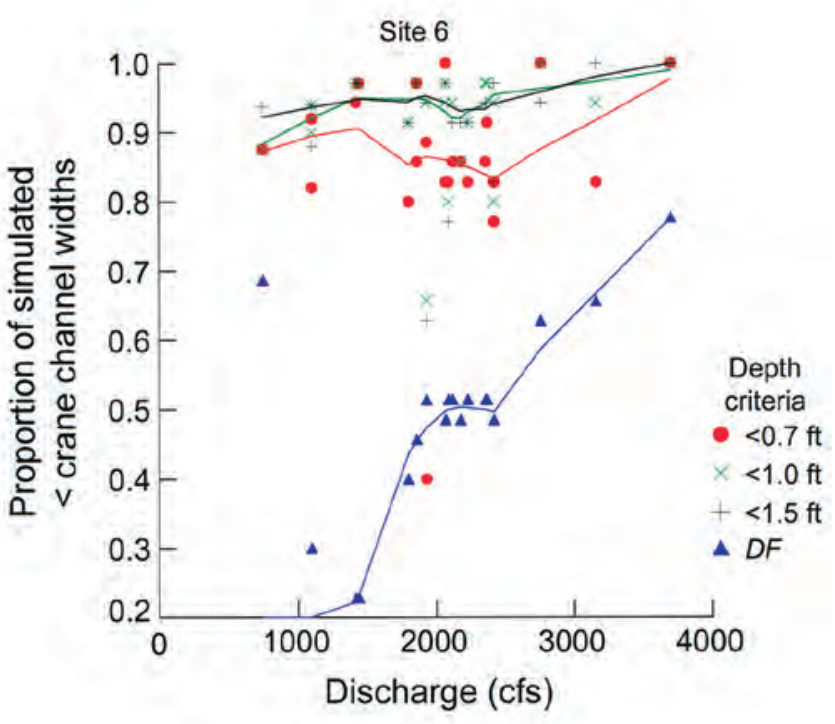

Figure 13. Proportion of simulated channel wetted widths less than or equal to the wetted widths used by cranes based on three maximum depth criteria (minimum depth $=0$, buffer $=0$ ) and the DF in segment 6 . Lines are lowess smoothing functions fitted through the respective data points.

$P<0.0001$ ), with the variation in proportionate differences increasing with increasing discharge as estimated by $10^{\text {th }}$ and $90^{\text {th }}$ regression quantiles (fig. 14). Nonrandom selection for the original depth function $(D F)$ was also evident (MRPP, $P$ $<0.001$ ) but the proportionate differences (median $=0.12$, range $=0.08$ to 0.42 ) were an order of magnitude smaller than for any of the simple depth criteria (e.g., $\Delta W W<0.7$ median $=$ 1.07 , range $=-0.58$ to 4.68$)$ and had little relation with discharge as indicated by the $10^{\text {th }}$ and $90^{\text {th }}$ regression quantiles (fig. 14). This analysis lends additional support to considering one of the alternative depth criteria (wetted channel widths $<0.7,<1.0$, or $<1.5$ foot depth) for describing suitability of channels for roosting cranes. Although we limited this analysis to segment 6 where most cranes were observed, thus providing sufficient samples for performing statistical tests, a similar pattern was expected in other segments. A specific choice of alternative depth criteria cannot be based solely on the preceding evaluation with Whooping Crane data. Additional considerations for selecting a specific depth criterion are discussed in more detail in the final section of this report.

\section{An Evaluation of Crane Roost Observations versus Discharge}

\section{An Alternative to the Habitat Model}

The modeling effort since the early 1980's has been directed at developing a tool that could be used to help support decisions about instream flows in the Platter River. The

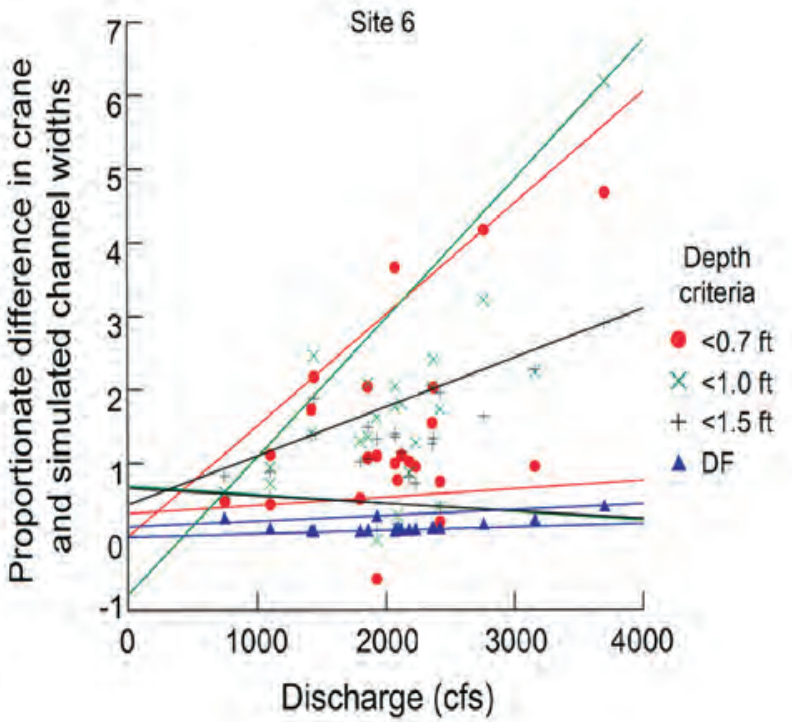

Figure 14. Proportionate differences in wetted channel widths used by cranes and the weighted average wetted widths from simulations for three maximum depth criteria (minimum depth = 0 , buffer $=0$ ) in segment 6 . The $90^{\text {th }}$ (higher lines) and 10th (lower lines) regression quantiles are shown.

various versions of the habitat model have included variables such as depth and wetted width that are not only thought to be important to cranes, but which also provide a functional linkage between habitat and discharge in a manner that allows one to explore the potential effects of flow variation. However, if Whooping Cranes really are affected by changes in discharge in the Platte River, it may be for other reasons that are not related to depth and wetted width per se. In other words, crane habitat suitability may be causally related to river discharge, but not in ways that have been perceived by model builders. Hence, we evaluated the Whooping Crane data to see if there was any evidence that crane sightings are related to discharge in a general sense.

\section{Methods and Results}

We examined the daily flow records for 4 March-8 May, corresponding to the dates of earliest and latest observations of Whooping Cranes during spring migration, for the Grand Island and Kearney-Odessa hydrologic reaches for the 23 years comprising 1980-2002. Days on which cranes were observed, number of sightings, and numbers of cranes were identified in the flow record.

We determined whether the total flows (or equivalently mean flows) over the migration period for the 11 years cranes were observed were likely just a random subset of all 23 years by conducting an exact permutation analysis with the excess group option of multiresponse permutation procedures, MRPP (Mielke and Berry, 2001:32-36). There was some indication that total flows in years when cranes were observed were an unlikely random subset in the Kearney-Odessa hydrological 
reach as cranes were only observed in 2 (1980 and 1987) of the 7 years with highest total flows (fig. $15 ; P=0.054$ ). Similarly for the Grand Island hydrologic reach, cranes were only observed in 1 (1998) of the 7 years with highest total flows (fig. 16; $P=0.019$ ). This could occur either because cranes tend to not stop on the Platte River during years of higher spring flows or because they are more difficult to detect under those conditions. The highest numbers of cranes $(11,10$, and 7) were observed in years $(1992,1996$, and 1997) with mean daily flows during the migration period of 1,600-2,200 cfs.

Platte River flows on days cranes were observed were compared against flows on all days within the migration period pooled across all years using the excess group option of MRPP to determine if the flows on days when cranes were observed were likely just a random subset of all available daily flows. There was weak evidence that the flow on days cranes were observed $(n=17)$ in the Kearney-Odessa hydrologic reach were a nonrandom subset of daily flows (MRPP, $P=$ 0.095), with most of the difference due to less use of daily flows $>2,200$ cfs than were available (fig. 17). Evidence that flow on days cranes were observed in the Grand Island hydrological reach $(n=15)$ were a nonrandom subset of daily flows also was weak (MRPP, $P=0.123$ ) with minor differences

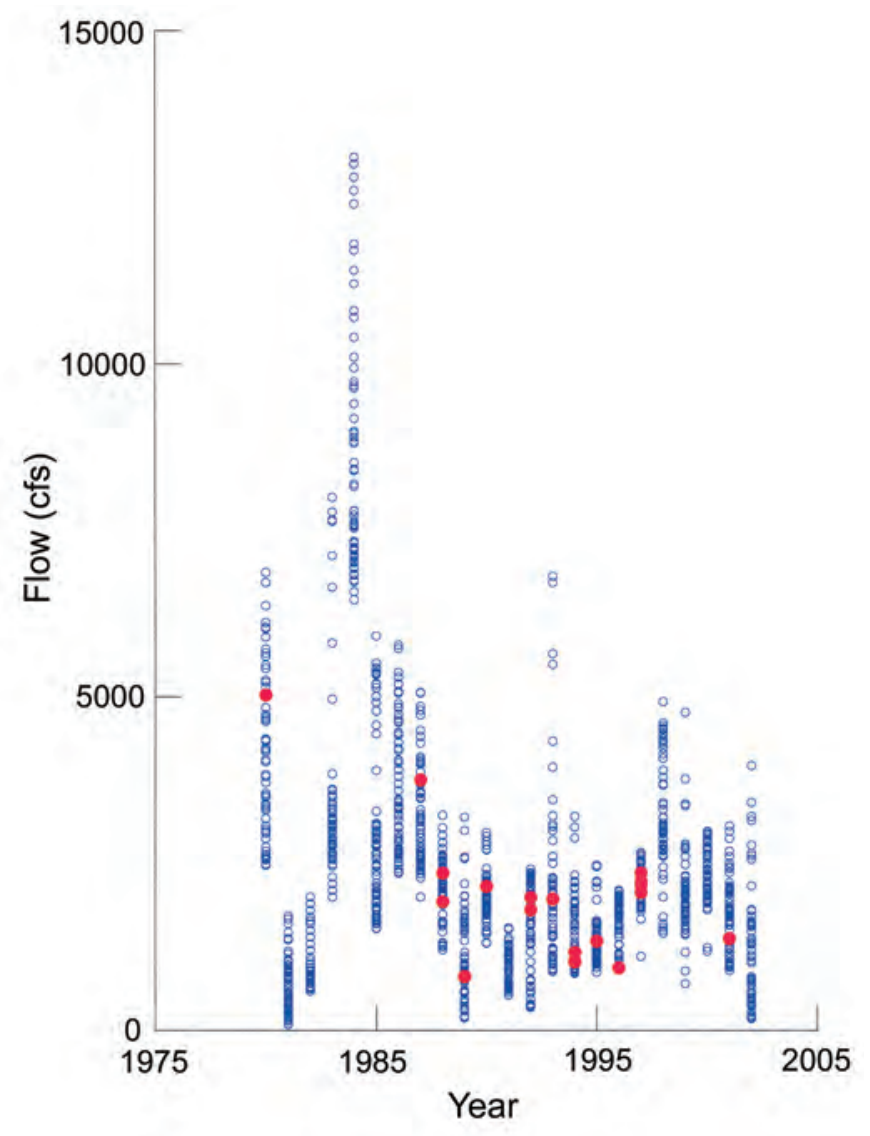

Figure 15. Daily flow (cfs) from 4 March-8 May in the KearneyOdessa hydrologic reach of the Platte River for 1980-2002. Red circles are days Whooping Cranes were observed.

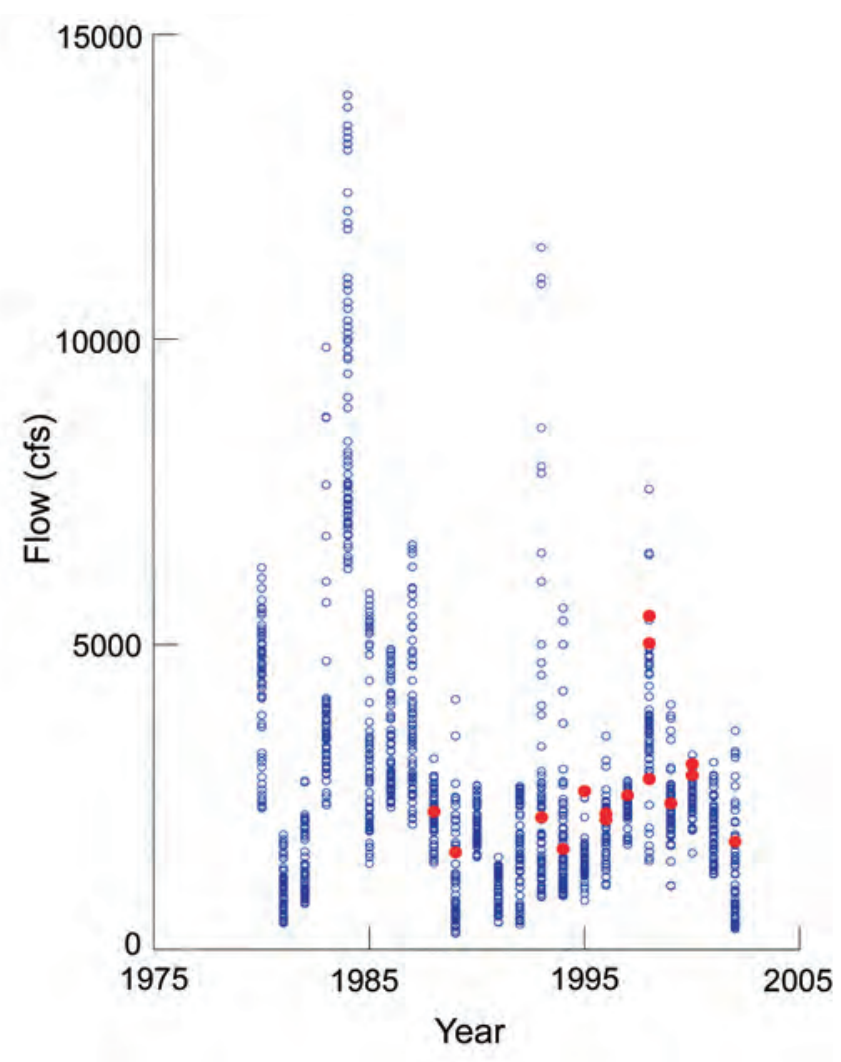

Figure 16. Daily flow (cfs) from 4 March-8 May in the Grand Island hydrologic reach of the Platte River for 1980-2002. Red circles are days Whooping Cranes were observed.

occurring because cranes made greater use of daily flows $1,500-2,200$ cfs than were available (fig. 17).

The daily flow analyses above ignored the great differences in flow during the migration period among years when cranes were observed and that in some years cranes were not observed. To examine relationships between flows on days cranes were observed adjusting for yearly differences in flow during the migration period, we regressed the flow on days cranes were observed (y) against the median flow for the migration period in the year and hydrologic reach where the crane was observed (X). Systematic departure from a 1:1 relationship across median seasonal flows was examined to determine if there was a deviation that might suggest some nonrandom selection. A 0.50 linear quantile (median) regression model was estimated and quantile rank score tests (Cade and others, 1999) were used to construct confidence intervals and test if the relationship was different from 1:1 $\left(\beta_{0}=0\right.$ and $\beta_{1}=1$ ). Pooled across hydrologic reaches, there was no statistical evidence that flow on days cranes were observed were other than just random deviations from a 1:1 response to median seasonal flows [flow on day crane was observed $=$ $-377.82(95 \% \mathrm{CI}=-1537.2$ to 794.7$)+1.230(95 \% \mathrm{CI}=0.59$ to 1.66) $\mathrm{x}$ median seasonal flow; $P=0.109$ for $\mathrm{H}_{0}: \beta_{0}=0 ; P=$ 0.149 for $\left.\mathrm{H}_{0}: \beta_{1}=1\right]$. Scatter plots and separate regression model estimates for each hydrological reach suggested some 

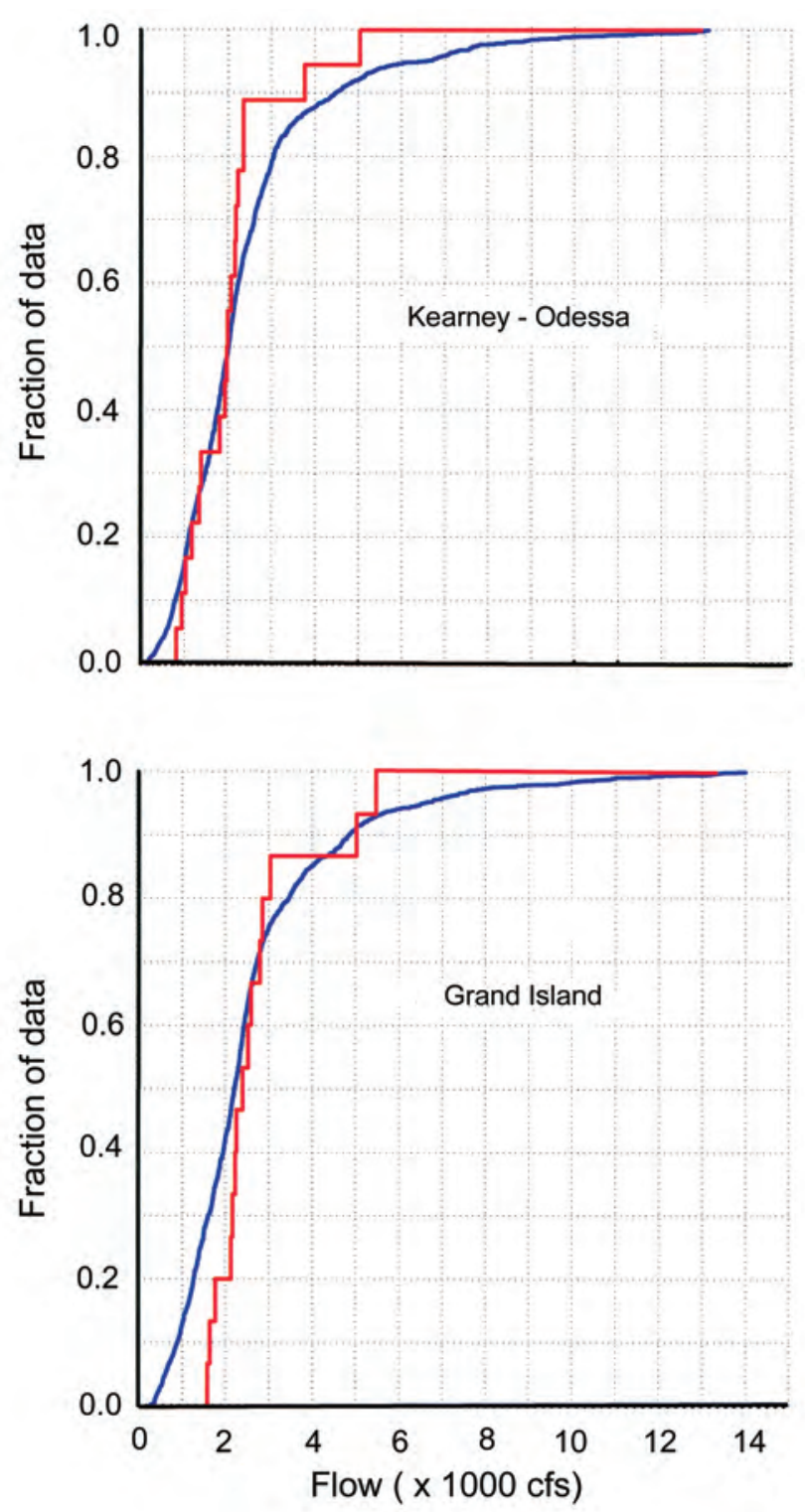

Figure 17. Cumulative distributions of daily flows (cfs) 4 March-8 May in the Kearney-Odessa and Grand Island hydrologic reaches of the Platte River for 1980-2002 (blue lines) and for days Whooping Cranes were observed (red lines).

evidence that flow on days cranes were observed in the Grand Island hydrologic reach were higher than the median seasonal flows when flows were $<1,800 \mathrm{cfs}$ (fig. 18). Because of the large variation in the Grand Island hydrological reach data, there was no statistical evidence to support different linear relationships between the Grand Island and Kearney-Odessa hydrologic reaches $(P=0.704)$. It is important to note that in years when median daily flows were $<3,000 \mathrm{cfs}$, the flow for 24 of 28 days cranes were observed deviated only slightly above and below the 1:1 relation with median daily flow, consistent with random selection of flow (fig. 18).

We also examined time series plots of daily flows between 4 March and 8 May in each hydrologic reach for

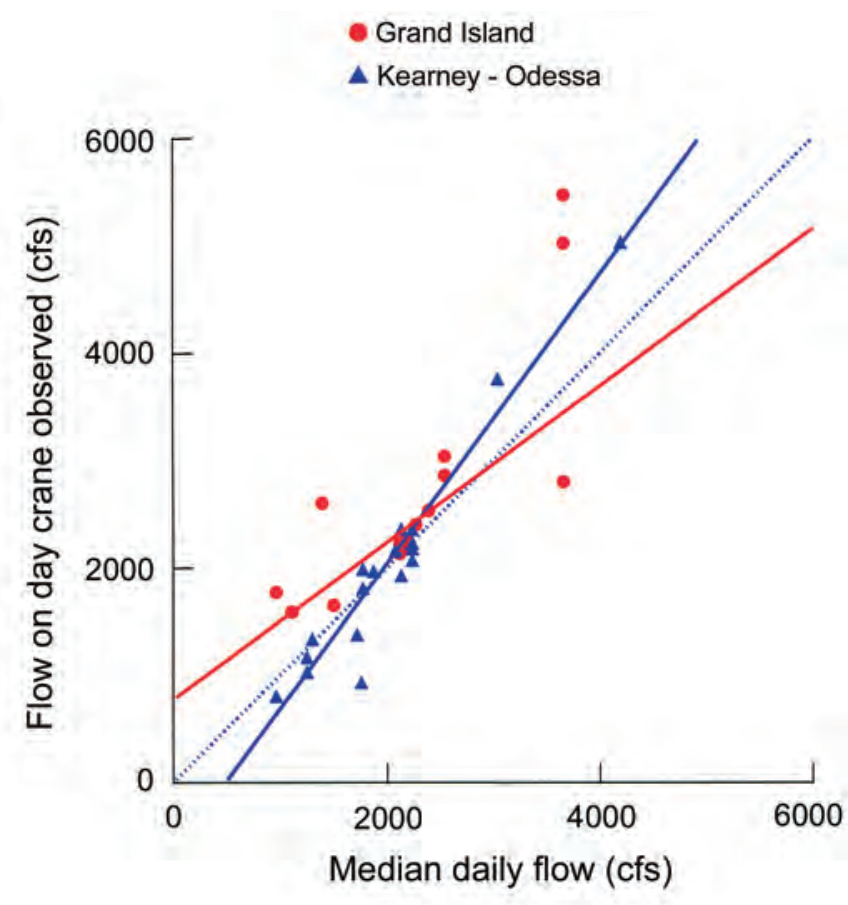

Figure 18. Flow (cfs) on days Whooping Cranes were observed by median daily flow 4 March-8 May at Kearney-Odessa and Grand Island hydrological reaches on the Platte River 1980-2002. Median regressions estimated for Kearney-0dessa: $n=18 ; b_{0}=-662.51$ $(95 \% \mathrm{Cl}=-1567.9$ to 18.8$), b_{1}=1.35(95 \% \mathrm{Cl}=0.95$ to 1.51$)$; and Grand Island: $n=15 ; b_{0}=774.84(95 \% \mathrm{Cl}=-2473.9$ to 1899.9$), b_{1}=$ $0.73(95 \% \mathrm{Cl}=0.33$ to 2.17$)$. Dotted line is 1:1 relation.

each year cranes were observed. Flow on days cranes were observed within these time series exhibited no obvious consistent pattern relative to peak, lowest, rising, or declining flows (figs. 19 and 20).

\section{Discharge-Habitat Relationships}

\section{Background}

One of the principal uses of the Whooping Crane habitat model is to estimate the relationship between discharge and the amount of roosting habitat within the Platte River channel. The general approach that has been used to derive discharge-habitat relationships involves two steps: (1) develop a discharge versus WUA relationship for each river segment, and (2) sum these relationships to get a single, aggregate relationship for the entire study area. Apart from the hydrologic issues associated with summing segment WUA's (see Hydrological Reference Points), this approach is complicated by the fact that there is not a single discharge versus WUA relationship for each of the study sites.

Study sites were visited for data collection on as many as nine different occasions. A separate WUA versus discharge 

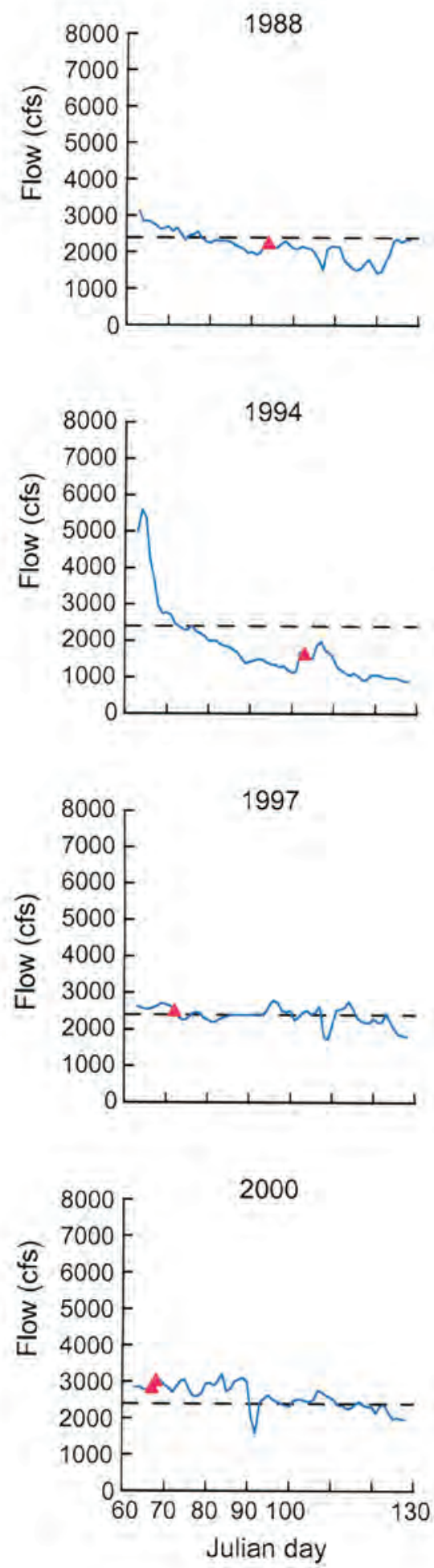
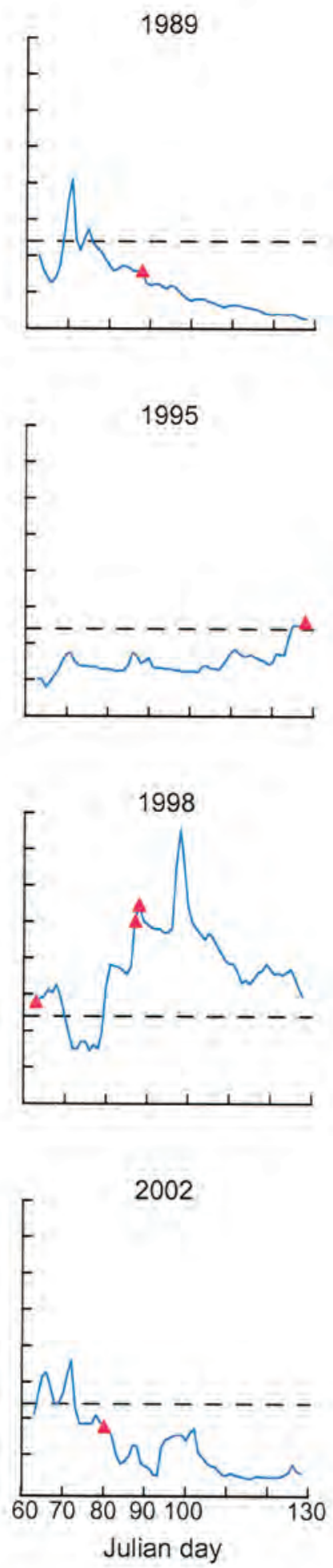
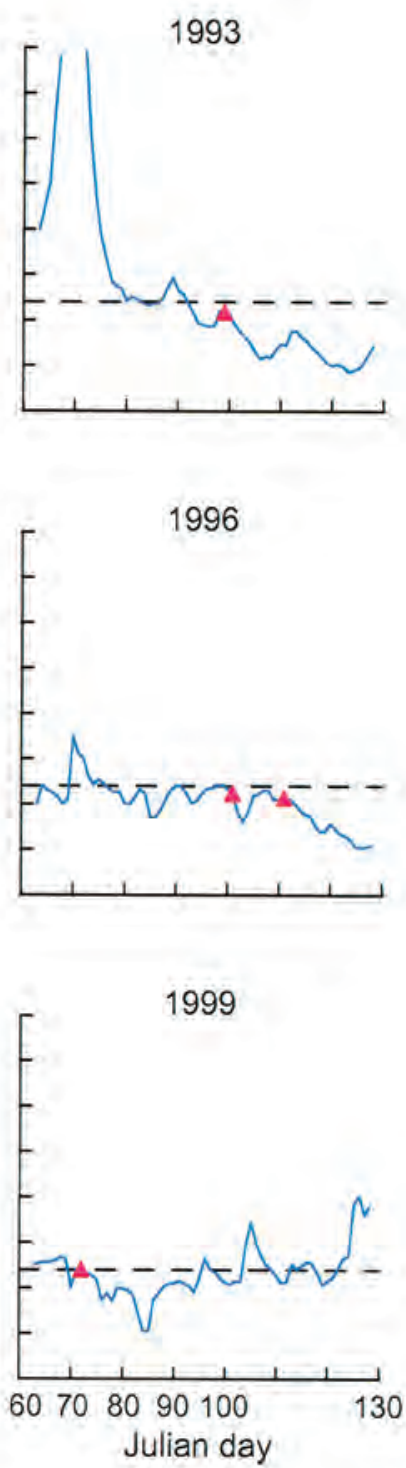

Figure 19. Whooping Crane observations (triangles) and daily flow (cfs) records from 4 March-8 May in the Grand Island hydrologic reach of the Platte River. 

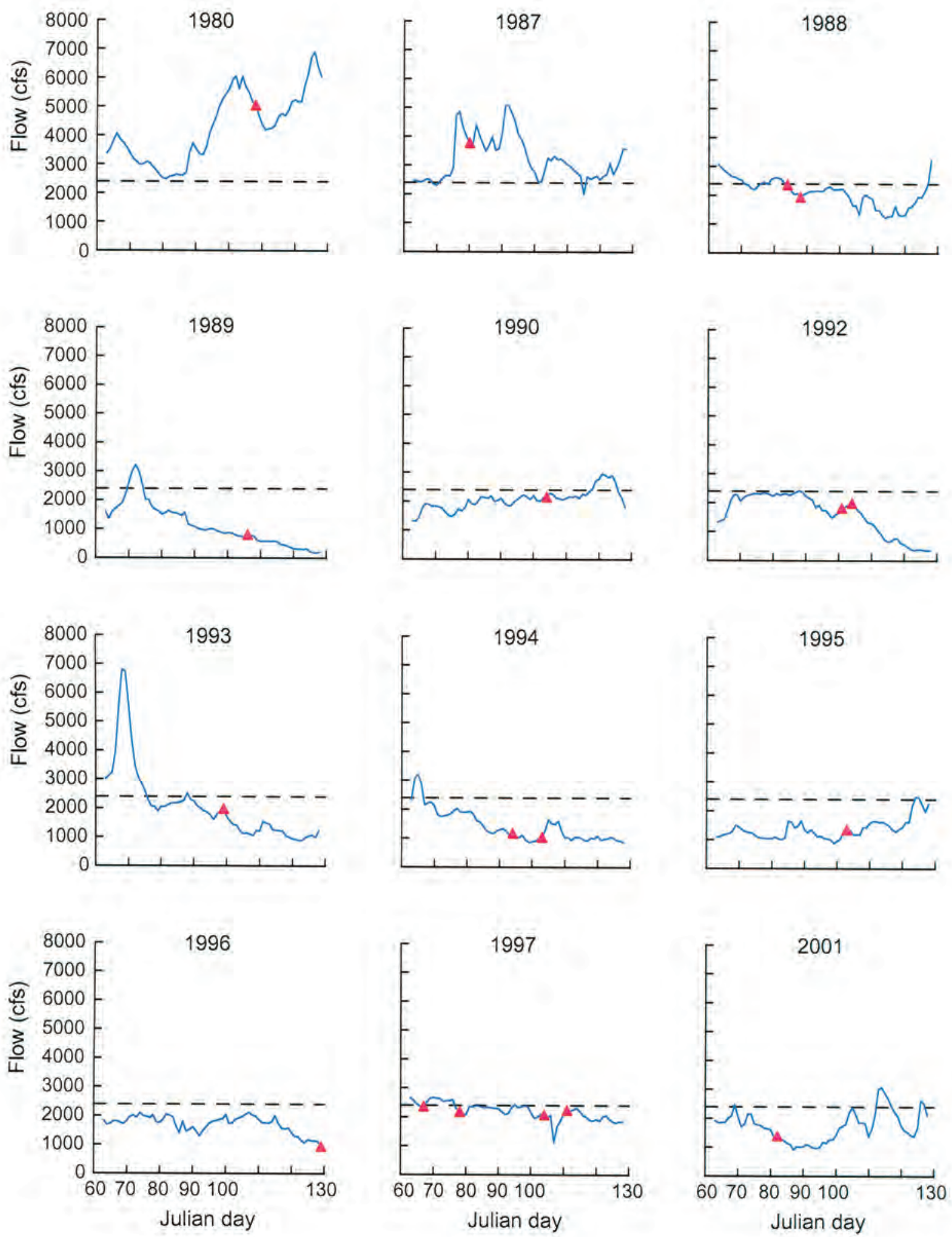

Figure 20. Whooping Crane observations (triangles) and daily flow (cfs) records from 4 March-8 May in the Kearney-0dessa hydrologic reach of the Platte River. 
relationship was developed for each visit (see Collecting and Processing IFG4 Data Files), based on measurements that were acquired during each visit to the site. The relationships derived from separate visits often cover a slightly different range of flows. However, even for discharges where the separate relationships overlap, there is some variation (fig. 21). There are multiple causes of this variation including measurement error, modeling error, and changes in channel morphology that occurred between visits.

The consequence of this inherent variation is that there is uncertainty in the relationship between discharge and WUA of Whooping Crane roosting habitat. It cannot be said with certainty, for example, that there are " $x$ " WUA units of habitat at a discharge of 2,000 cfs, nor can it be said that the optimum habitat conditions occur at precisely the discharge of $\mathrm{Q}(\mathrm{cfs})$. Even if hydraulic and channel topography data could be collected with no measurement error (which they cannot), and even if the hydraulic models were perfect in simulating water surface elevation (which they are not), there would still be some uncertainty because the Platte River channel is dynamic, and habitat conditions change over time. The issue, therefore, is determining how to contend with this variability in establishing management goals and performing impact assessments for alternative flow scenarios.

\section{The Spline Approach}

The historical approach for dealing with this inherent variability was to fit a single curve through the data points for each study site (fig. 21), and then to conduct habitat analyses with the assumption that the single curve accurately depicted reality. There are many different algorithms that could be used to fit the WUA versus discharge data, and each of these would give different results. For example, one statistical program alone, SYSTAT version 9.0, offers more than 120 curvefitting options (Wilkinson, 1999). Previous analyses utilized a particular spline program from the software library on the U.S. Bureau of Reclamation's mainframe computer in Lakewood, Colorado. There is very little available documentation for the program; the mainframe computer and software library no longer exist. We were provided two different versions of the FORTRAN code, neither of which could be run successfully. The written documentation provided to us contained a source listing for yet a third version of the spline program. Using the written documentation and two versions of the FORTRAN code, we were able to develop a fourth version that runs and produces results similar to those reported in Carlson and others (1990).

This spline program has one trait that may have guided its choice for the Whooping Crane analysis: it forces the curve through data points representing measured discharges. These points were perceived to be very important in defining the overall relationship. Each field visit to a study site produced cross sectional data that were input into program Water Surface Profile (WSP) (Milhous and others, 1984) to estimate the water surface elevations across a range of flows
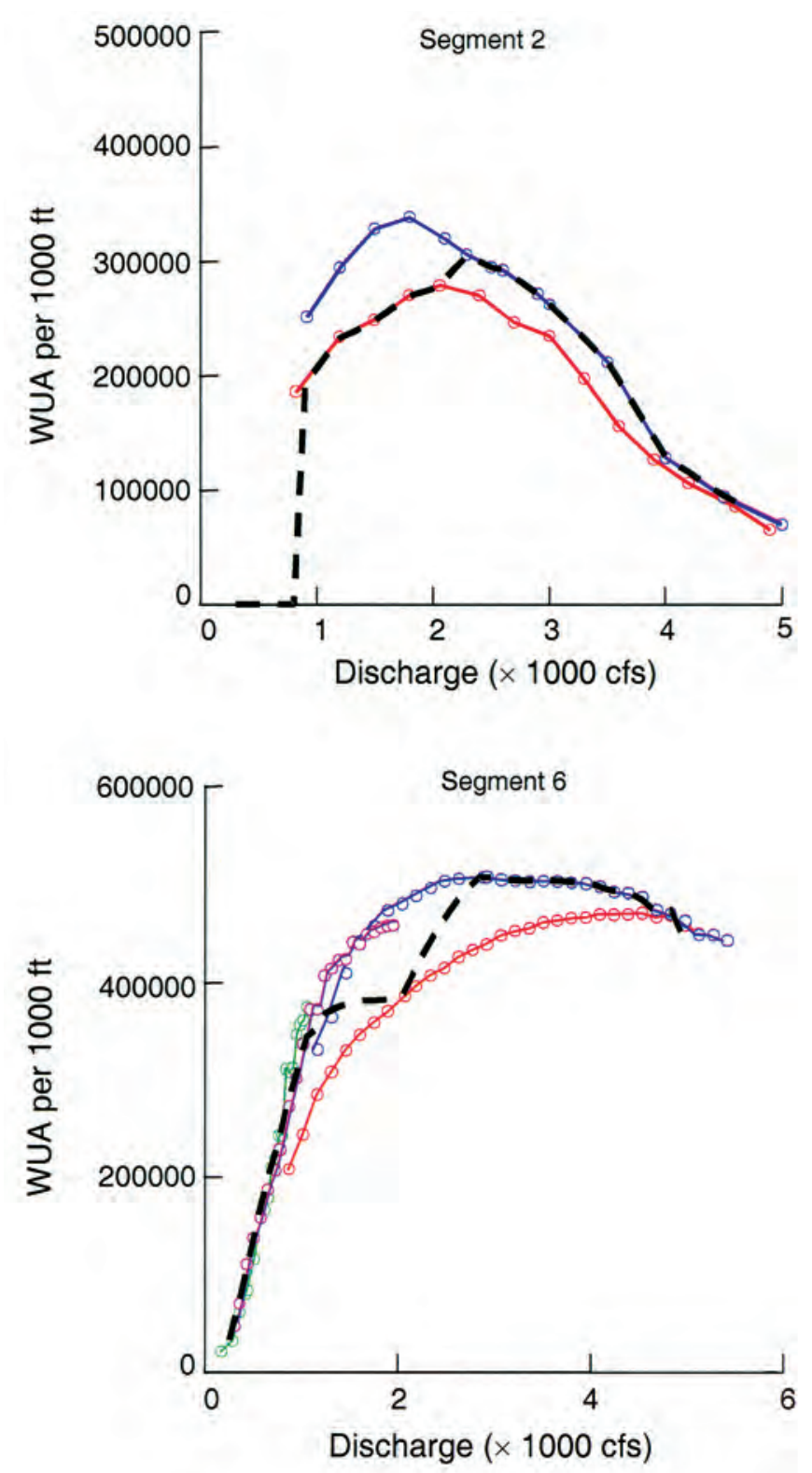

Figure 21. Different WUA versus discharge relationships for study sites 2 and 6 . Study site 2 was visited twice (red = visit 1; blue = visit 2), and study site 6 was visited four times (red $=$ visit 1 , blue $=$ visit 2 , green $=$ visit 3 , purple $=$ visit 4 ). The dashed lines are the spline curves that were fitted through the data using program SPLINE.

roughly between 0.4 and 2.5 times the flow measured during the site visit. This range represents the domain within which the hydraulic model is believed to be reasonably accurate (Bovee and Milhous, 1978). There is some (unknown) model error associated with the estimates at each of the simulated flows, but the data point representing the measured flow is free of model error because the water surface elevation at this flow was actually measured during the site visit. Thus, 
habitat estimates associated with the measured flows were assumed to be higher quality data, and the spline program was configured so that the relationship passed through these points. This approach produces a curve that takes erratic jumps at the measured points (fig. 21).

There are two flaws with this spline approach, however. First, the data points representing measured flows are not error free, and therefore there is no justification for forcing the relationship through these points (Robert Milhous, USGS-Fort Collins Science Center, oral commun., 2000). Even if the river channel were static (which it is not) and one repeatedly measured a site at a given flow, the estimated WUA would still vary because of systematic and measurement error. Furthermore, the channel is not static, but instead changes through time, introducing additional variation in the estimated WUA at any given discharge. Hence, the measured points are not an exact measure of WUA at the time of measurement, much less an exact estimate of the expected WUA in the future. Second, the use of a single curve masks the uncertainty inherent in the discharge-habitat relationship. There is really a range of WUA values associated with any single discharge; management recommendations for a specific discharge to meet a habitat goal may be unnecessarily restrictive. Masking the inherent uncertainty in the data exposes future management recommendations to unnecessary criticism that can be avoided simply by recognizing the inherent variability in the habitat-discharge relationship.

\section{An Alternative Randomization Approach}

We developed and implemented an alternative approach for deriving discharge-habitat relationships that is, we believe, technically superior because it avoids the previously identified problems. This approach is outlined here in general terms, and it is implemented in the Microsoft Windows program developed for the Service (see Computer Programs). The randomization approach described herein has the advantage that the inherent system variability is acknowledged and can be utilized to develop prediction limits for estimates of WUA. This approach is intuitively simple and works as follows:

1. For each study site, define the range of values for WUA at each of several selected flows. Beginning at $100 \mathrm{cfs}$, for example, and proceeding to 5,000 cfs in steps of 50 cfs $(100,150,200, \ldots, 5,000 \mathrm{cfs})$, WUA is estimated with each of the separate WUA relationships, and a maximum and minimum value of WUA is defined for each flow. The range of 100-5,000 cfs is arbitrary, but spans the range of flows of concern for assessing crane habitat. If these maximum/minimum values are plotted, they form a band that includes all the measured and simulated data points at a site (fig. 22).

2. Repeatedly resample between the minimum-maximum values, defined above, to estimate WUA statistics (e.g., mean, variance, and range) at each specified flow.
There are different ways to implement this resampling process, depending on the particular question or management issue being addressed. Two examples are given here based on habitat model C4R.

\section{Example 1. Estimating the Discharge-Habitat Relationships for a Single River Segment}

For this example, we chose segment 6, which is represented by flow data collected at study site 6 on four different occasions. First, we defined the range of WUA values from the four site visits, for individual flows between 100 and 5,000 cfs. Then beginning at $100 \mathrm{cfs}$, we randomly selected 1,000 WUA values (assuming a uniform distribution) between the minimum and maximum values. This process was repeated every $50 \mathrm{cfs}$ between 100 and 5,000 cfs. Thus, we produced a data file with 1,000 different estimates of WUA for each of 100 different discharges from 100 to 5,000 cfs. We used these data to produce basic statistics on WUA by flow (table 6). The 'mean' values, as well as the $95 \%$ confidence limits on the estimated mean values, which would prove helpful in management planning, are shown graphically in fig. 22 for study site 2 .

\section{Example 2. Estimating the Optimal Flow for Multiple River Segments}

The same procedure can be used to aggregate WUA outputs across two or more river segments (the Windows program described in the next section follows this procedure), as demonstrated by the following example. Beginning at

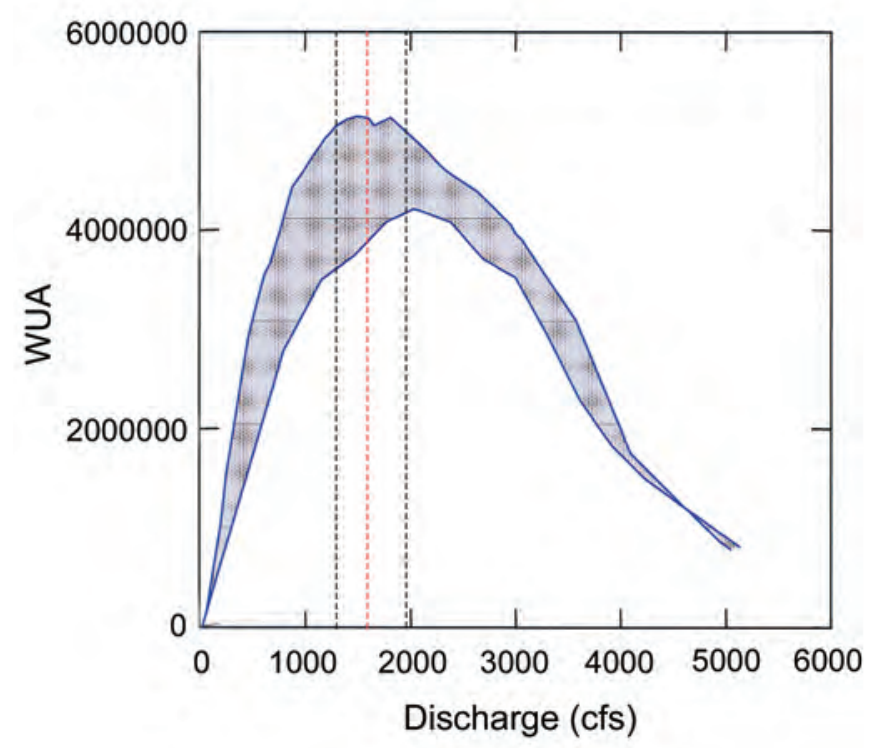

Figure 22. The range of WUA (crosshatched band) for discharges in study site 2 . The red vertical line is the optimal discharge 11,600 cfs), and the dashed lines are the $95 \%$ confidence limits on the optimal discharge (1,300-1,950 cfs) estimated with randomization. 
$100 \mathrm{cfs}$, we randomly selected a WUA value (between the minimum and maximum value) for each study site, and these WUA values were added to get a total WUA for $100 \mathrm{cfs}$. We repeated this every $50 \mathrm{cfs}$ up to and including 5,000 cfs. The result was a single curve for WUA versus discharge across the range of discharges. We then repeated this curve-building step 1,000 times to produce 1,000 different WUA versus discharge curves. Next, we inspected each of the 1,000 relationships to identify the discharge that maximized WUA (the 'optimal' flow), and saved these 1,000 estimates of optimal flow. We computed the median value, the range of optimal values for all 1,000 simulations, and the range of values for the central $95 \%$ of the simulations (i.e., the $95 \%$ prediction interval (PI)).

We repeated this procedure for the entire study area (Sites 2-12B), the segments comprising critical habitat (Sites 2-7, $8 \mathrm{c}$ ), and for each individual study site. The resulting statistics

Table 6. Weighted usable area statistics for river segment 6 based on resampling $(n=1,000)$ the field data collected during four separate visits to study site 6 .

\begin{tabular}{|c|c|c|c|}
\hline \multirow[b]{2}{*}{ Discharge (cfs) } & \multicolumn{3}{|c|}{ Weighted usable area } \\
\hline & Mean & S.D. & Range \\
\hline 400 & 3,759 & 380 & $3,097-4,405$ \\
\hline 600 & 6,421 & 312 & $5,896-6,977$ \\
\hline 800 & 9,264 & 696 & $8,047-10,457$ \\
\hline 1,000 & 12,804 & 1,485 & $10,170-15,351$ \\
\hline 1,200 & 14,432 & 1,190 & $12,394-16,481$ \\
\hline 1,400 & 15,899 & 1,268 & $13,734-18,173$ \\
\hline 1,600 & 16,866 & 1,203 & $14,799-18,914$ \\
\hline 1,800 & 17,771 & 1,266 & $15,549-19,886$ \\
\hline 2,000 & 18,480 & 1,247 & $16,761-20,575$ \\
\hline 2,200 & 19,051 & 1,190 & $17,018-21,048$ \\
\hline 2,400 & 19,645 & 1,100 & $17,629-21,485$ \\
\hline 2,600 & 19,981 & 1,034 & $18,169-21,771$ \\
\hline 2,800 & 20,242 & 934 & $18,645-21,848$ \\
\hline 3,000 & 20,427 & 820 & $19,068-21,858$ \\
\hline 3,200 & 20,607 & 673 & $19,433-21,742$ \\
\hline 3,400 & 20,687 & 588 & $19,636-21,693$ \\
\hline 3,600 & 20,810 & 517 & $19,905-21,720$ \\
\hline 3,800 & 20,860 & 467 & $20,048-21,667$ \\
\hline 4,000 & 20,869 & 428 & $20,122-21,599$ \\
\hline 4,200 & 20,804 & 326 & $20,244-21,369$ \\
\hline 4,400 & 20,728 & 274 & $20,263-21,206$ \\
\hline 4,600 & 20,569 & 185 & $20,246-20,873$ \\
\hline 4,800 & 20,272 & 46 & $20,191-20,354$ \\
\hline 5,000 & 19,866 & 58 & $19,785-19,985$ \\
\hline
\end{tabular}

for optimal discharges are reported in table 7. Each study site peaks at a slightly different discharge depending on the hydraulic characteristics of the site. Generally, the most upstream (i.e., the lower numbered) study sites tend to have narrower channels; thus, they peak at a lower discharge. The optimal flow for the critical habitat (1,800 cfs; $95 \%$ PI $=1350$ to $2,400 \mathrm{cfs}$ ) is somewhat lower than that for the entire study area $(2,400 \mathrm{cfs} ; 95 \% \mathrm{PI}=2,000$ to $2,950 \mathrm{cfs})$ because the critical habitat is on the upstream end of the area.

This randomization approach represents a departure from the traditional method of fitting a particular type of spline through the data. The randomization approach does not, in and of itself, produce a single graphical relationship between discharge and habitat as has been used in the past (e.g., Carlson and others, 1990). Instead, the emphasis in randomization is to describe statistical relationships between discharge and habitat while also accounting for the inherent uncertainty in the data. This is a more robust, defensible basis for performing impact assessments and management planning for the Platte River because it does not require one to ignore variability in the data and therefore to treat the river as a static system.

\section{Computer Programs}

\section{Documentation of Computer Code}

We were provided electronic copies of the FORTRAN source code for three computer programs that the Service used historically to implement the Whooping Crane habitat model. Two of these programs, PREFORW and WHOPCWC, were described in some detail in a file report (Ziewitz, 1987). Documentation of the third program, SPLINE, consisted of a 2-page undated, anonymous report provided by Dave Carlson, U.S. Fish and Wildlife Service. The function of the computer programs is summarized below (fig. 23).

The programs PREFORW and WHOPCWC were designed to work in tandem, and a DOS batch program, WHOOPER, was also developed to run PREFORW and WHOPCWC for a specified IFG4 data file and version of the habitat suitability criteria. A single run of PREFORW and WHOPCWC produced a data file containing the coordinate points for WUA versus discharge for the range of discharges simulated in the IFG4 data file. Hence, a user of these programs had to use PREFORW-WHOPCWC repeatedly to get WUA-discharge estimates for all the visits to a study site (fig. 23). After PREFORW-WHOPCWC had been run for each visit to a site, the program SPLINE integrated the data from all visits to produce a single relationship of WUA versus discharge for the study site. Program SPLINE was run separately for each of 16 study sites, resulting in 16 separate data files containing the coordinate points for the individual site spline curves (e.g., Appendix D; Carlson and others, 1990). The 16 data files were then imported into a commercially 
Table 7. Statistics on optimal discharges estimated form 1,000 simulations with habitat suitability model C4R. Prediction intervals could not be determined for some study sites with limited data. Values reported are total discharge at the Grand Island Gauge.

\begin{tabular}{|c|c|c|c|}
\hline \multirow[b]{2}{*}{ Study site } & \multicolumn{2}{|c|}{ Optimal discharge } & \multirow[b]{2}{*}{ 95\% P.I. } \\
\hline & Median & Range & \\
\hline 2 & 1,600 & $1,400-2,300$ & $1,100-2,250$ \\
\hline 3 & 1,700 & 1,700 & 1,700 \\
\hline $4 \mathrm{~A}$ & 1,300 & 1,600 & $950-1,400$ \\
\hline $4 \mathrm{~B}$ & 1,400 & 1,400 & 1,400 \\
\hline 5 & 1,700 & 1,700 & 1,700 \\
\hline 6 & 3,050 & $2,400-4,300$ & $2,550-3,950$ \\
\hline 7 & 1,450 & $1,000-1,600$ & $1,150-1,600$ \\
\hline $8 \mathrm{AN}$ & 2,000 & 2,000 & 2,000 \\
\hline $8 \mathrm{AS}$ & 1,050 & $600-1,400$ & $700-1,350$ \\
\hline 8B & 2,000 & $1,300-2,700$ & $1,550-2,500$ \\
\hline $8 \mathrm{C}$ & 1,650 & $1,200-2,700$ & $1,250-1,900$ \\
\hline 9BE & 3,000 & $2,400-3,700$ & $2,600-3,400$ \\
\hline $9 \mathrm{BW}$ & 2,850 & $2,100-4,000$ & $2,400-3,700$ \\
\hline 10 & 3,500 & $2,600-4,000$ & $3,050-3,850$ \\
\hline 11 & 1,900 & $1,300-2,100$ & $1,700-2,050$ \\
\hline $12 \mathrm{~A}$ & 3,050 & $2,000-3,300$ & $2,500-3,250$ \\
\hline $12 \mathrm{~B}$ & 2,000 & 2,000 & 2,000 \\
\hline $\begin{array}{r}\text { Critical } \\
\text { habitat } \\
\text { (sites } 2-7, \\
8 \mathrm{C})\end{array}$ & 1,800 & $1,100-2,600$ & $1,350-2,400$ \\
\hline $\begin{array}{r}\text { Study } \\
\text { area (sites } \\
002-12 \mathrm{~B})\end{array}$ & 2,400 & $1,800-3,100$ & $2,000-2,950$ \\
\hline
\end{tabular}

available spreadsheet program and aggregated to produce a single curve (e.g., fig. 2; Carlson, 1994) for the entire study area (Dave Carlson, oral commun., 1999).

Running this set of programs for the entire Whooping Crane study area requires 54 separate runs of PREFORWWHOPCWC and 16 separate runs of SPLINE. Additionally, some data files had to be manipulated by a user between these computer runs and for the final development of an aggregate spline curve. The learning curve was steep for effectively running these programs and the Service no longer had knowledgeable staff to perform these duties when we began this study. Consequently, the programs could not be run, for example, to assess the consequences of suggested modifications to the habitat suitability model.

The Service asked us to review the programs and revise them to make them functional and more user-friendly. Thus, a large portion of our initial effort in this project was learning the FORTRAN code, getting the programs to run successfully, and attempting to replicate the earlier results that were achieved with the programs.

Generally, there was good documentation of the FORTRAN programs. Programs PREFORW and WHOPCWC were well written and contained sufficient comments to allow one to understand the program flow. Program SPLINE, however, was an exception in that its documentation was virtually non-existent. We were provided two different versions of the source code (labeled SPLINE and WTAVE), but neither of these versions would execute correctly using outputs from WHOPCWC. Moreover, we acquired a source listing of the SPLINE program attached to the 2-page anonymous report mentioned earlier, but this source listing was for yet a third version of the code for which we had no electronic copy. Thus, we started our analysis with three different versions of the same program, with no documentation of which version 


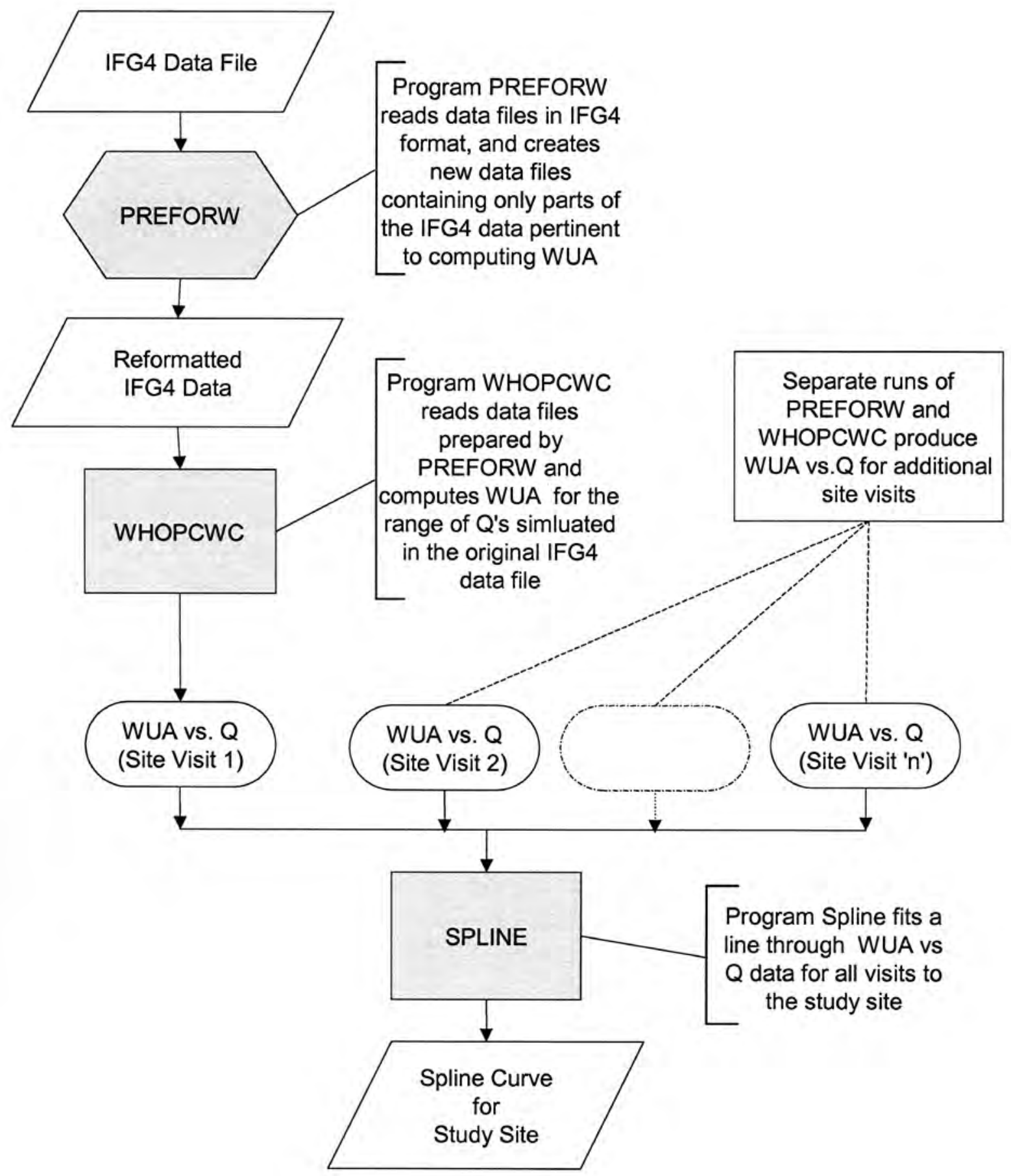

Figure 23. A flow diagram of the relationships between FORTRAN programs PREFORW, WHOPCWC, and SPLINE. 
was used in the most recent habitat analyses performed by the Service.

\section{Verification of Computer Code}

We compiled PREFORW, WHOPCWC, and SPLINE using Lahey's (v. 4.5) FORTRAN 90 compiler. This recompilation allowed us to run each program in Debug mode, stepping through the computations one line at a time to verify the calculations and logic flow. No significant problems (i.e., problems that affected the habitat calculations) were detected in either PREFORW or WHOPCWC. However, several apparently inconsequential problems were found and corrected to prevent future problems from cropping up. For example, program PREFORW had a "Y2K-like" problem having to do with the vegetation/habitat codes recorded for each station along a cross section. This code allowed only two digits, and in those cases where the habitat code exceeded 99, its value was truncated. Thus, when PREFORW read a code of 100 it converted this to 00 .

Program SPLINE was problematic. Using the three, non-functioning versions of the code, we rewrote the program and finally succeeding in getting it to run using outputs from WHOPCWC. However, we had no good standard with which to judge whether the program was giving the correct results. The only available standard consisted of the spline curves for each of the 16 study sites in Carlson and others (1990). The program as we rewrote it generally reproduces those figures, but there are differences that we could not explain with the information at our disposal.

\section{Enhancements to the FORTRAN Programs}

We made several changes to the individual programs with two objectives in mind: (1) modifying data output files to have a consistent record structure so that the files could readily be imported into other applications (e.g., spreadsheet, statistical programs); and (2) adding additional variables to some data files to facilitate coupling the programs together in an automated fashion. Specific enhancements included the following:

1. PREFORW was modified to read the measured flow on the 'CAL1' line and to read the study site number (new variable SEGID) and visit number (new variable OBSID) from the header of an IFG4 file.

2. PREFORW writes the measured flow, SEGID, and OBSID out to tape 2 for later use by WHOPCWC and other programs.

3. SPLINE was revised to read data files produced by WHOPCWC, rather than keyboard inputs and screen prompts.

4. SPLINE output formats were modified to allow the curve coordinates to be imported directly into various plotting programs, and the curves are based on WUA for the river segments represented by each study site, rather than WUA/1,000 feet for the study site as in the original code.

5. We developed a new program, ADDSPLIN, which aggregates the spline curves described above to produce a single curve for the entire study area.

6. Several new programs were developed to assist with developing discharge-habitat relationships using the randomization method described in the previous section, DISCHARGE-HABITAT RELATIONSHIPS. These first of these new programs, MINMAX, defines the range of WUA for selected discharges between 100 and 5,000 cfs for each study site. The second new program, RESAMPLE, samples from the range of WUA produced by MINMAX, and produces statistics that are used to characterize the WUA versus discharge relationship for each study site. Listings for all these FORTRAN programs are provided in Appendix E.

\section{A Microsoft Windows Program}

A Microsoft Windows program, Platte River Habitat Analysis, was partly built upon the earlier FORTRAN programs. These earlier programs, PREFORW and WHOPCWC, were designed to analyze the data from a single visit to a study site/segment, hence running the programs for the entire Whooping Crane study area required up to 54 separate runs, including manual manipulation of intermediate data files. Consequently, the programs could not be quickly rerun, for example, to assess the consequences of different scenarios. Moreover, there was no feasible way to conduct sensitivity analyses on various model parameters (e.g., suitability relationships).

The goals for development of the Platte River Habitat Analysis program were to make the Whooping Crane model and associated programs much more user friendly by:

1. Automating the application of the Whooping Crane suitability model so that all river segments are automatically analyzed in a single run.

2. Providing an easy means for the user to change model parameters, rerun applications, and conduct sensitivity analyses.

3. Providing a direct link to the OPSTUDY (Rodney, 2002) hydrology model so that different water use scenarios can be easily assessed in terms of Whopping Crane habitat.

4. Making application of the model much more user friendly by providing a graphic user interface, similar to familiar Windows applications. 
5. Building a solid foundation for future expansion of the program to include other types of analyses, data displays, or suitability criteria for other species.

Currently the program allows a user to EDIT suitability criteria, segment lengths, and IFG4 files, and the ANALYSES menu provides two different types of habitat analysis. The basic functionality of the program is outlined here.

\section{Edit-Suitability Criteria}

This window allows one to change the habitat suitability criteria. All aspects of the habitat suitability model can be modified in this window (fig. 24). The choices are:

1. Depth Criteria. Choose either the depth function $(D F)$ supported by the C4R model (Carlson, 1994), or simple depth constraint. With the simple depth constraint, only depths between a specified minimum and maxi- mum depth are suitable $(\mathrm{SI}=1.0)$, and all other depths are unsuitable $(\mathrm{SI}=0.0)$.

2. Depth Constraints. Specify a minimum and a maximum depth. These constraints are used for both criteria above-the $D F$ historically has been computed for depths between 0 and 3.5 feet.

3. Obstructions. The suitability of a river cross section is affected by width of 'shallow' (defined by the above depth constraints) channels. However, otherwise suitable wetted channel within the "buffer" of a visual obstruction may not be used by Whooping Cranes, and hence should not be included in the calculation of wetted width. The default value is 0 (zero).

4. Wetted Width Curve. The suitability curve for wetted width can be described using up to 6 coordinate pairs. The default curve is: $(0,0.0),(120,0.0),(850,0.9)$, $(1,000,1.0)$, and $(5,000,1.0)$.

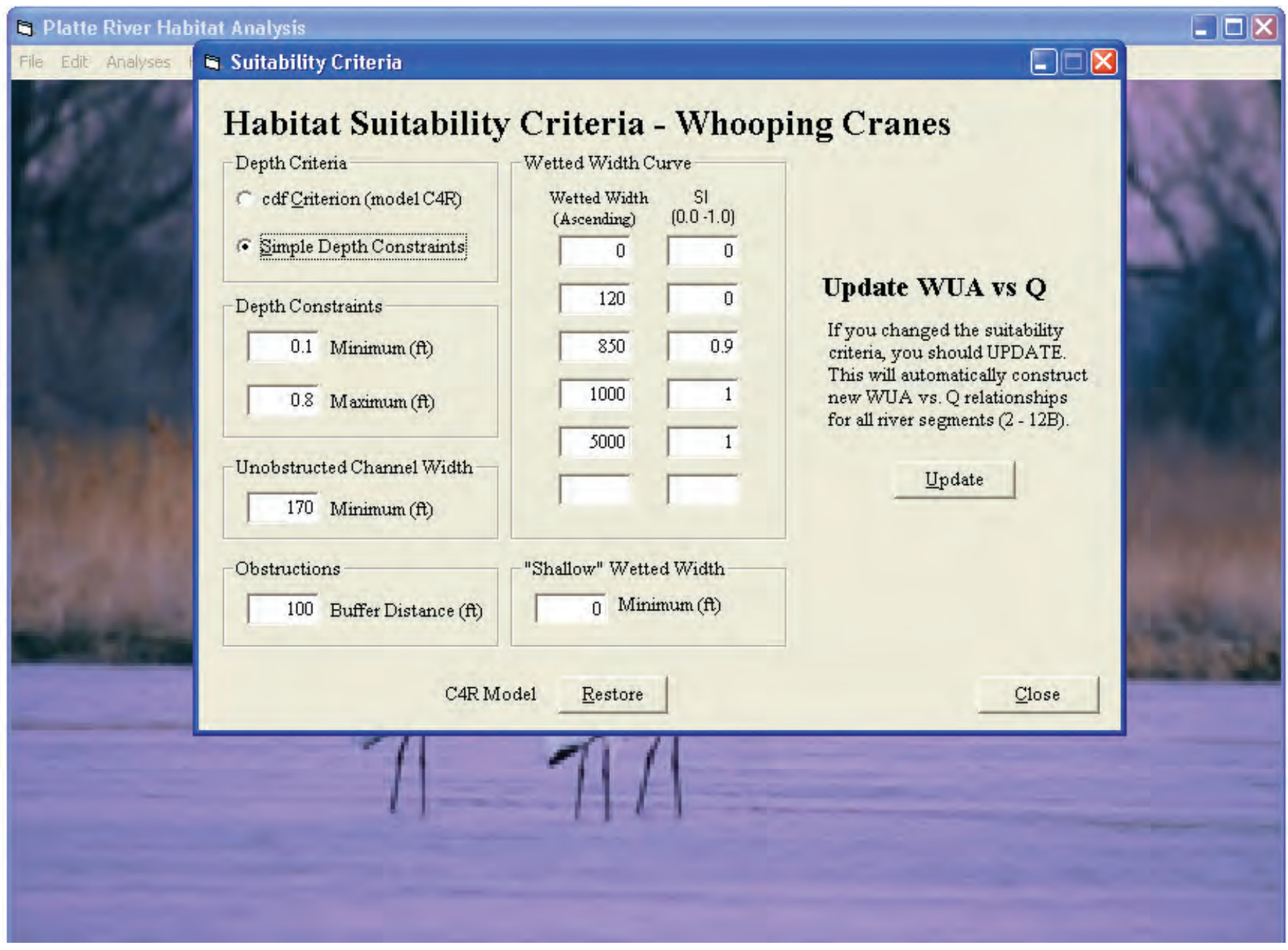

Figure 24. The Habitat Suitability Criteria screen in the Platte River Habitat Analysis program allows the user to change model parameters to explore the consequences of using different model assumptions. 
5. Unobstructed Channel Width. Any unobstructed channel width less than this amount is automatically assigned a suitability of 0.0 (zero). The default is 170 feet.

\section{Edit-Segment Lengths}

The segment lengths (default values) are the undisturbed segment lengths (Appendix B) taken from Farmer and others (2000; Appendix 1). Any or all these values may be changed to reflect changes in disturbance along the river. Zero is an acceptable value, and can be used to effectively remove a segment, or isolate a segment in the analyses (fig. 25).

\section{Edit-River Segment Data}

The program provides a set of screens to view segment maps, to view water surface profiles for cross sections at each site visit, and to plot WUA versus discharge relationships for currently defined site visits. This set of screens also allows users to import new IFG4 data as they become available.

Presently, the ANALYSES menu provides two types of habitat analyses: (1) estimate the optimal discharge, which is the discharge that would maximize WUA, and (2) assess future WUA associated with a scenario defined by outputs from the OPSTUDY model.

\section{Analyses-0ptimum Flow}

When the 'Run' button is clicked, the program estimates the discharge that would maximize the WUA by segment and across the entire study area. The computations follow the randomization process outlined in the previous section. Beginning at $100 \mathrm{cfs}$, a random WUA value (between the minimum and maximum value) is selected for each river segment, and these WUA values are added to get a total WUA for $100 \mathrm{cfs}$.

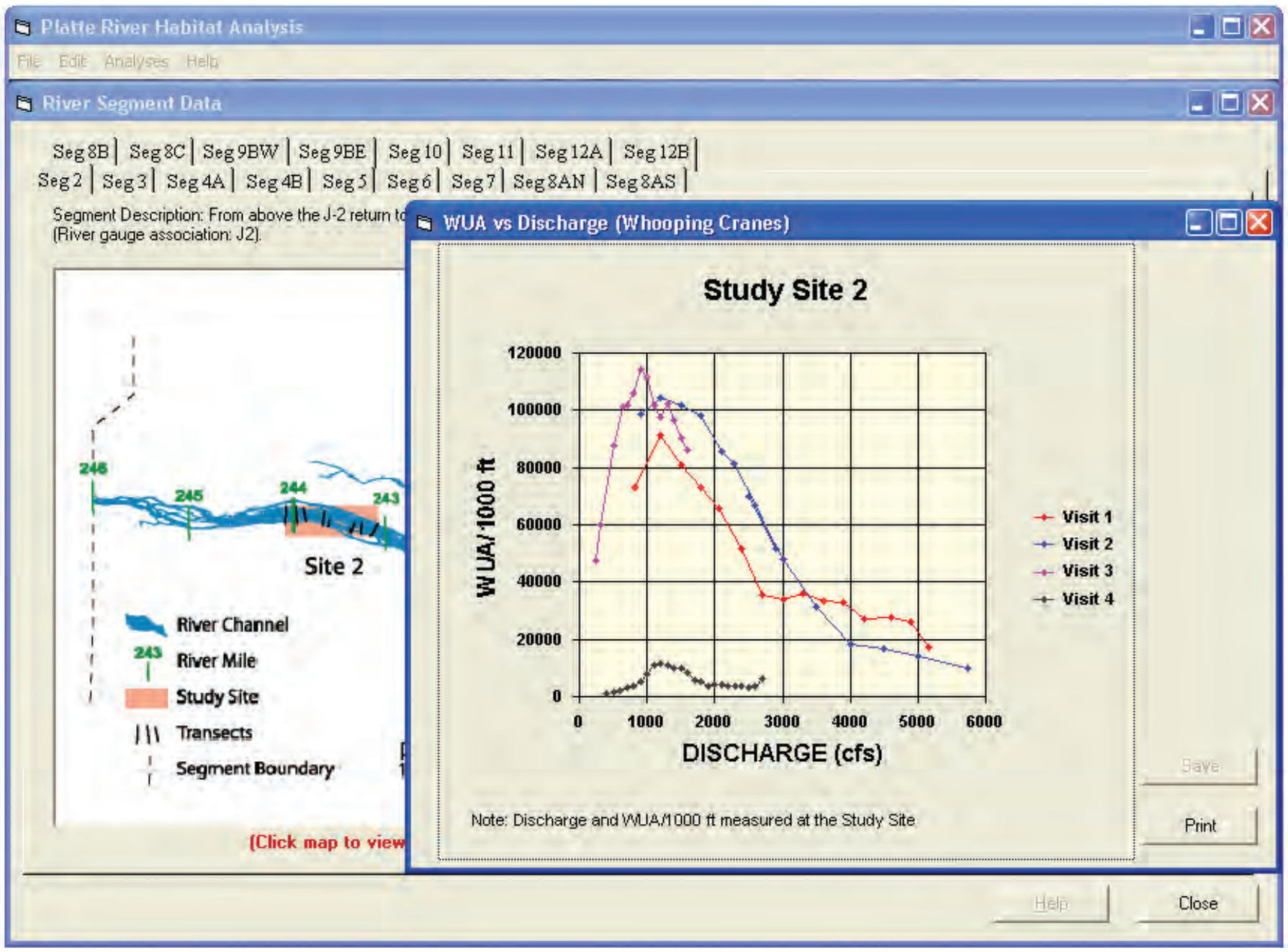

Figure 25. The River Segment Data Screen allows the user to visualize each segment by seeing a segment map, viewing water surface profiles for each cross section, and plotting WUA versus discharge relationships for all study site visits. New IFG4 files can be imported when they become available. 
This is repeated every $50 \mathrm{cfs}$ up to and including 5,000 cfs. The discharge at which the maximum summed WUA occurs is saved to a file and the above process is repeated 1,000 times. From the 1,000 values for optimal discharge, the median value and $95 \%$ confidence limits are reported as for a single segment. The results can be viewed in tabular and graphic form.

\section{Analyses-Assessment with OPStudy}

This analysis option was designed to estimate future WUA associated with scenarios previously analyzed by the hydrology program OPSTUDY (or files with a format similar to those from OPSTUDY). The habitat assessment is conducted with hydraulic files representing four distinct reaches along the Platte River: 'J2Flows.txt' represents the J2 Return gauge, 'OVFlows.txt' represents the Overton gauge, 'ODFlows.txt' represents the Odessa gauge, and 'GIFlows. txt' represents the Grand Island Gauge. If you click Browse, search for and open an OPSTUDY file (with file extension .tab), the four data files above are automatically extracted from the opened OPSTUDY file (tables 54, 56, 135, and 138).

When the 'Run' button is clicked discharges for the selected month are extracted from the appropriate OPSTUDY file for each river segment. The discharges are translated to the corresponding range of WUA for each segment. A random WUA is drawn for each year, between the minimum and maximum for that year, and a curve of WUA versus year is produced. The WUA versus year curve is integrated (WUA is totaled across all years for which WUA was predictable), and the total is divided by the number of years for which WUA was predictable (based on the WUA versus Q relationships for each segment). The resulting statistic is called 'average annual WUA' or AA-WUA. The above steps are repeated 1,000 times resulting in 1,000 values for AA-WUA. The median value is reported as AA-WUA, and the $25^{\text {th }}$ and $975^{\text {th }}$ values are reported as the $95 \%$ confidence limits. Results are available in tabular and graphic (fig. 26) form.

\section{Discussion}

\section{What Have We Learned About the Habitat Model?}

Models are simplifications of real systems, and consequently will never be without some shortcomings and limitations. Instead of expecting perfection, one should judge a model against its alternatives with regard to which alternative best achieves the stated purpose of the model. Throughout its development, a recurring theme in the Whooping Crane habitat model has been that channel width is a very important determinant of habitat suitability. The $\mathrm{C} 4 \mathrm{R}$ model reflects the generally accepted concept that channel widths, both unob- structed and wetted widths, are important habitat variables. Moreover, our evaluation of the C4R habitat model shows that cranes tend to use river segments that, on average, have greater unobstructed channel widths, and tend to select the wider, wetted cross sections within a segment.

The habitat model, however, was developed to do more than quantify channel widths from the perspective of Whooping Cranes. The model was developed, and has been applied for: (1) setting instream flow targets for the Whooping Crane (U.S. Department of the Interior, 1994); (2) assessing flow depletion impacts for biological opinions regarding ESA, section 7 consultations with other federal agencies; and (3) evaluating alternatives in the Platte River Recovery Implementation Program Draft Environmental Impact Statement. These applications require that the habitat model describe the relationship between river discharge and Whooping Crane roosting habitat suitability, and this in turn requires that the model framework incorporate a variable pertaining to water depth in the channel.

Our evaluation shows that the C4R's water depth variable, the depth function (DF) based on cumulative frequency distributions of depths at 21 crane roosts, has serious logic flaws, is a weak predictor of habitat suitability, and is outperformed by alternatives that are based on simple depth criteria. But which alternative depth criteria should be used?

\section{A Workshop to Discuss Depth Criteria}

Alternative depth criteria were discussed at a workshop held at the offices of the Whooping Crane Habitat Management Trust near Grand Island, Nebraska on February 13-14, 2001 (Farmer and others, 2001). The authors, Service staff from the Grand Island and Denver, Colorado offices, and several Whooping Crane authorities (Jim Lewis, U.S. Fish and Wildlife Service (retired); Brian Johns, Canadian Wildlife Service; John Dinan, Nebraska Game and Parks Commission; Craig Davis, Oklahoma State University; and Gary Lingle, University of Nebraska, Lincoln) attended this workshop. Many of the statistical analyses presented in this report were first discussed at that workshop. Alternatives considered in detail were based on replacing the depth function with a much simpler variable such as the wetted width of channel $<0.7$ feet depth, or the wetted width of channel $<0.7$ feet depth and also more than 80 feet from a visual obstruction. Similar variables were, in fact, used in earlier versions of the model. We considered 18 different alternative depth criteria with different ranges of suitable depths and buffer sizes (table 5).

There was considerable discussion following the presentations on the various evaluations of depth criteria. There was a consensus that the simple depth criteria, such as in table 5, seemed to be the appropriate way to model suitability, but there was no consensus which specific depth criteria, including criteria not identified in table 5, was best. The concept of using the model to identify the discharge that maximizes the amount of suitable roosting habitat (defined as having water between the maximum and minimum depth) was questioned, however. 


\section{All River Segments}

\section{$\rightarrow$ Anпual WUA $\quad$ - Average Anпual WUA}

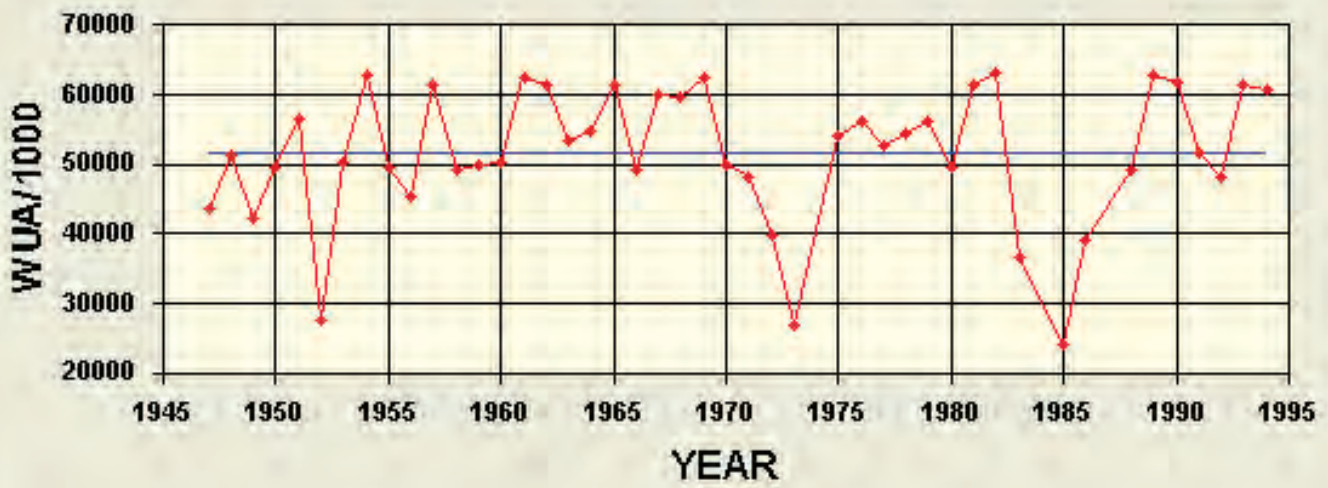

Plot Segment?

r $\operatorname{seg} 2$

C $\operatorname{seg} 3$

r $\operatorname{seg} 4 A$

(5) Seg $4 \mathrm{~B}$

C $\operatorname{seg} 5$

C $\operatorname{seg} 6$

C $\operatorname{seg} 7$

C Seg BAN

(c) Seg 8AS

r $\mathrm{seg} 8 \mathrm{~B}$

C Seg 80

r seg 9BW

C Seg $9 B E$

C $\operatorname{seg} 10$

r seg 11

r $\operatorname{seg} 124$

r $\operatorname{seg} 12 \mathrm{~B}$

c All Segments

Scenario $=$

Figure 26. The output window produced by a run of the Whooping Crane model with hydrological simulations produced by program OPSTUDY. The user can receive tabular and graphic (shown here) results.

When using the alternative depth criteria such as in table 5 , it may not be desirable to maximize the total amount of river channel within the specified depth range, per se. Whooping Cranes appear to be roosting on the "tops" of inundated sandbars, and it was suggested that they seldom roost in troughs even if the water depths are correct according to the model criteria. Hence, the appropriate management strategy might be to use the model to identify the discharge that maximizes the amount of inundated sandbar "tops." Further discussion led to the realization that this would require some operational definition of a "top". Even if such an analysis were conducted, however, it was not clear if the maximizing flow would differ substantially from the flow that would maximize the total amount of area within defined depth constraints.

The discussion of sandbar tops led to a discussion of the two-dimensional, landscape context of individual roost sites. Those who had conducted Whooping Crane aerial surveys have noticed that roost sites are clearly visible as relatively large, inundated "macroforms." Thus, it appears that cranes may select roost sites not based on depth alone, but also based on the presence of an inundated sandbar that has a minimum area, width, or breadth. Furthermore, a suitable macroform may need to be located a minimum distance away from the banks, as well as from perceived obstructions both up- and downstream. There was discussion about how one might use the existing crane roosting data to evaluate these landscape factors, and it was concluded that the existing data were not sufficient for that purpose.

\section{Analysis of Wetted Widths versus Discharge}

During the workshop, Mike Armbruster of the U.S. Bureau of Reclamation described an analysis that had been conducted by Reclamation aimed at understanding the overall relationship between discharge, channel morphology, and habitat suitability. This analysis looked at the discharge-depth relationship for Sandhill Crane roosting habitat at eight sites located from below (downstream) the J-2 return (study site 2) downstream to Chapman (Site 12A), and represented by multiple transects $(n=47)$ measured between 1984 and 1986.

Roosting area, represented by mean transect length within a 3-9" depth range, was maximized in a range of discharges between 800 and 1,600 cfs at these eight sites at measured flows between 1,068 and 2,062 cfs. The average discharge at which transect length in a 3-9" depth range was maximized was $1,175 \mathrm{cfs}$. The average transect length in the 
3-9" depth range was 328 feet at the eight sites. Roosting area, represented by the 3-9" depth range, is reduced as discharge increases. For example, at 2,400 cfs the mean transect length in the 3-9" depth range at four sites was 194 feet. All eight of the sites were resurveyed between 1998 and 2001, and illustrate a similar pattern. Roosting area (3-9" depth range) was maximized (381 feet) at $975 \mathrm{cfs}$ for measured flows between 653 and $1,750 \mathrm{cfs}$. At 2,400 cfs, four sites exhibited a mean transect length in the 3-9" depth range of 235 feet.

These simulated values can change with changes in measured flow at the sites, and with evaluation of different depths. However, a similar pattern remains. There is a range of discharges (700 to 1,600 cfs for the 3-9" depth range at surveyed sites) that occur after the channel first fills that maximize roosting area for Sandhill Cranes. The discharge at which the channel first fills varies at each site. When the mean transect data (1984 to 1986) for the eight sites discussed above are plotted (discharge versus wetted transect length), a few sites show a distinct flattening of the wetted width curve between 600 and 1,200 cfs. Such flattening in the curve occurs because wetted width increases sharply for initial increases in discharge, however the rate of increase in wetted width declines after the riverbed becomes fully wetted. At higher discharges, there is little remaining uninundated channel for the additional water to occupy.

This analysis was based on aggregation of transects from eight sites, and the channel filling discharge likely varies at each site based on channel width, depth, and bed morphology. Nevertheless, it seems likely that the channel filling discharge for individual sites is less than 1,500 cfs, substantially less than the Service's flow target of 2,400 cfs.

\section{Review by Whooping Crane Authorities}

In May 2002, a questionnaire was sent to four biologists familiar with crane usage of the Platte River (Dr. Craig Davis, Oklahoma State University; Dr. Jane Austin, USGS, Northern Prairie Wildlife Research Center; Dr. Felipe Chavez-Ramirez, Platte River Whooping Crane Management Trust; and Dr. Michael Armbruster, U.S. Bureau of Reclamation) by author Jeffrey Runge. The questions probed the appropriateness of using the C4R model's depth function versus alternatives including simple depth constraints discussed in the workshop (Farmer and others, 2001). The consensus of this review was as follows.

All four reviewers stated that simple depth constraints would be an appropriate alternative to the depth function of model C4R. An appropriate maximum depth was 0.8 feet. Austin and Richert (2001) recognized this depth as the $90 \%$ quantile for riverine roost sites. Some of the reviewers, however, believed that 0.8 feet was closer to a maximum threshold for suitability as opposed to an optimal depth. Based on information also from Austin and Richert (2001), the minimum depth constraint should be $>0.0$ feet (i.e., bare sand) because all observed roost sites were associated with wetlands. A depth of 0.1 feet was chosen to represent the minimum depth constraint, which was similar to shallowest observed depth of $5 \mathrm{~cm}$ (Austin and Richert 2001). Apart from the research cited above, there is little information on habitat selection on a micro scale, at the actual roost location. Hence all reviewers acknowledged that there is some uncertainty in these numbers.

All reviewers supported the concept of buffers because a shallow spot (i.e., between 0.1 and 0.8 feet depth) must also be located at a minimum distance from visual obstructions to be suitable for Whooping Cranes. There were no published data on this parameter value, so we estimated this distance based on the cross sections measured at Whooping Crane roost observations (Appendix D). The recorded distances to the nearest visual obstruction ranged from 80 to 575 feet $(n$ $=40$, median $=211$ feet), and the 25,75 and $90 \%$ quantiles were 320,133 , and 100 feet, respectively. To remain consistent with $90 \%$ quantile recommendation for maximum depth constraints, a 100 foot buffer would seem appropriate. In a sense, the C4R model already incorporates the concept of a buffer by specifying a minimum unobstructed channel width of 170 feet, or the equivalent of an 85 foot buffer from each bank.

\section{A Revised, Improved Model}

Several lines of evidence including: our statistical analyses, river hydraulic analyses conducted by U.S. Bureau of Reclamation, results from workshops held during the course of our work, and review comments from Whooping Crane authorities all point in the same direction-the depth function of model C4R should be replaced with a more supportable alternative. There is a consensus that suitable roosting habitat exists between about 0.1 and 0.8 feet of depth. If such alternative depth criteria were adopted, the revised model using the wetted width curve (fig. 2), a minimum unobstructed width of 170 feet, and a buffer of 100 feet around obstructions would predict an optimum flow for the study area as a whole of about 1,350 cfs (as compared to the current flow targets of $2,400 \mathrm{cfs}$ ). There, of course, would be some uncertainty in all components of this revised model, though less so than with the C4R model. To explore the possible consequences of this uncertainty, we conducted a sensitivity analysis on all model parameters, by varying one parameter at a time, while holding all others constant. A more complex, factorial analysis could, and probably should be conducted in the future by the Service.

During the development of model C4R, there was uncertainty in the value that should be selected for the minimum unobstructed width. Earlier versions of the model used 500 feet, but later versions settled on 170 feet because Whooping Cranes had been recorded roosting in channels this narrow. Hence, we varied the minimum width from its current value of 170 feet to as much as 400 feet, and recorded the model's predicted optimal flow for the entire study area. Changes in the minimum unobstructed width have very little influence, causing no more than about a $50 \mathrm{cfs}$ change in the model predictions (fig. 27). 
Conducting a sensitivity analysis of the wetted width curve (fig. 2) was potentially much more complex because one could vary the minimum suitable wetted width (assumed to be 120 feet), the point at which wetted width becomes optimal (1,000 feet), or the shape of the curve (the C4R model assumes a linear relationship between wetted width and channel suitability, but it has been suggested that this might be a non-linear relationship). For the analysis here, we simply varied the point at which the wetted width becomes optimal [from its assumed optimal value of 1,000 feet (fig. 2)] from 900 to 1,500 feet. The predicted optimal flow varied less than $50 \mathrm{cfs}$ over this entire range.

Buffers around visual obstructions have been recommended, although they were not included in the C4R model. Hence, we looked at the effects of varying buffer distance between 0 (no buffer) and 200 feet. The optimal flow without a buffer is predicted to be about 1,300 cfs. For an 80 foot buffer the optimal flows jumps to about $1,350 \mathrm{cfs}$, and for a 100 foot buffer the optimal flow is about 1,400 cfs. There was no more than about a $50 \mathrm{cfs}$ difference in optimal flows buffers between 100 and 200 feet (fig. 28). Hence, the decision about whether or not to include a buffer in the model would seem to have little consequence on model results.

Varying the minimum and maximum depth constraints had a somewhat larger influence on model predictions. Varying either of these parameters around their likely values has a similar effect, about a $100 \mathrm{cfs}$ change in the optimal flow for every 0.1 change in the parameter value (figs. 29 and 30). On the one hand, these effects are relatively large compared to the other parameters, so it may be important which specific values are chosen. On the other hand, there may not be that much actual uncertainty in the values from an ecological perspective, because there seems to be general agreement among crane biologists that the maximum depth is somewhere around 0.7 or 0.8 feet, and likely no more than 1.3 feet. Hence, with respect to the maximum depth, optimal flows may be about $1,350 \mathrm{cfs}$ (using 0.8 feet), but could be as high as $1,850 \mathrm{cfs}$ [using the

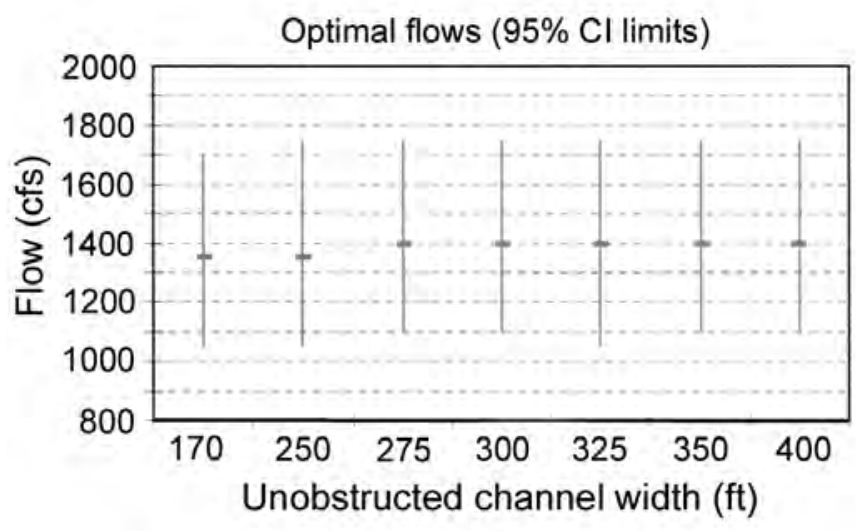

Figure 27. Variation in estimated optimal flow as a consequence of different assumptions about the minimum unobstructed width. Gray lines show the $95 \%$ confidence limits of estimated optimal flow.

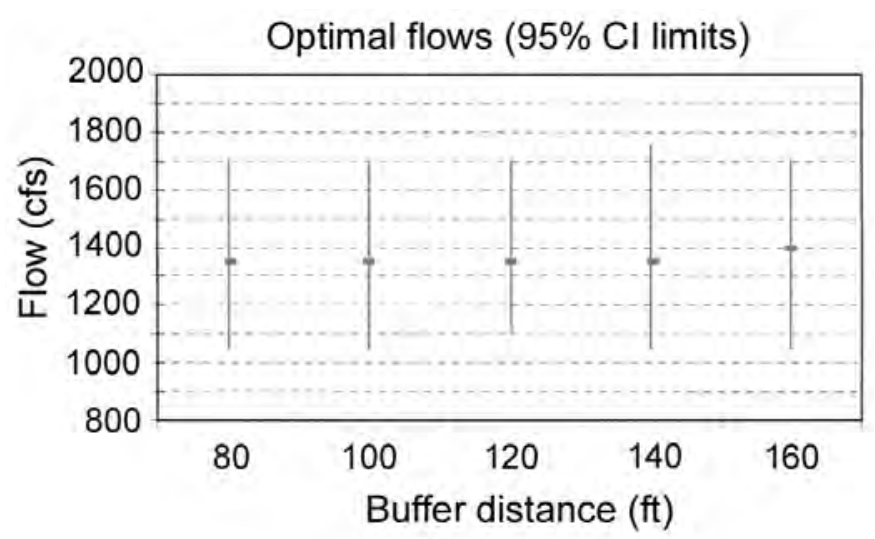

Figure 28. Variation in estimated optimal flow as a consequence of different assumptions about the buffer distance around visual obstructions. Gray lines show the $95 \%$ confidence limits of estimated optimal flow.

1.3 foot maximum observed depth for all riverine observations from Austin and Richert (2001)].

\section{Potential Model Extensions}

During our work, a number of other model issues were identified. Service and Reclamation biologists identified and discussed many of these in earlier workshops, and many of the issues relate to expanding the scope of the model to incorporate other factors, for perhaps achieving other purposes. We discuss them briefly below.

1.2-D Modeling. Horizontal visibility is regarded as an important aspect of Whooping Crane roost suitability. In the current model(s), unobstructed channel width serves as an index of horizontal visibility, but unobstructed channel width only measures visibility laterally (perpendicular to river flow). A better understanding between the relationship between lateral visibility and longitudinal visibility (upstream and downstream visibility) is needed for a better understanding of open horizontal visibility. A 2-D model would help with this understanding by accounting for changes in vegetation structure due to succession and habitat management.

2.Definition of an obstruction. Visual obstructions are defined as any channel bank or vegetation that is taller than $1 \mathrm{~m}$ in height. Visual obstructions are recorded along a roost site transect and serve as an index of horizontal visibility. There needs to be a better understanding of varying heights of obstructions, the distance of an obstruction from a roost site, and the direction of an obstruction from a roost site to better define an obstruction's effects on roost site suitability. Moreover, the new channel coding system for identifying obstructions should be revised with regard to the value of recently managed areas, to allow for improvements in 


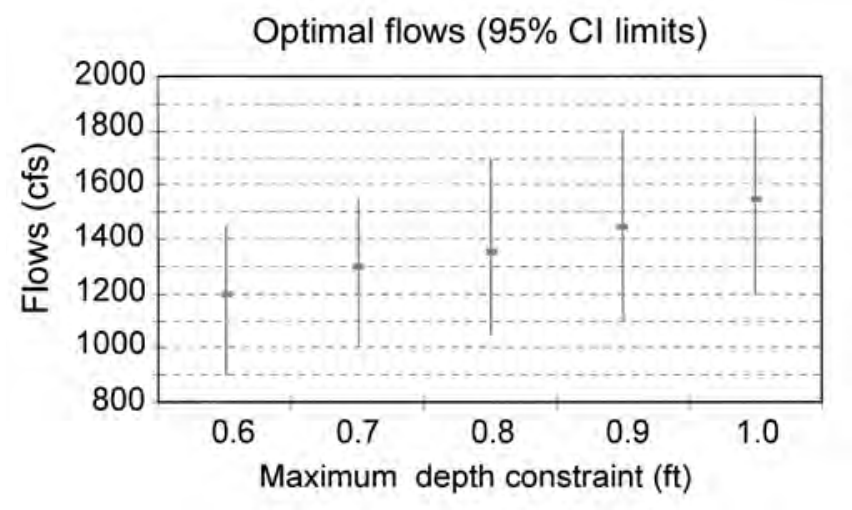

Figure 29. Variation in estimated optimal flow as a consequence of different assumptions about the maximum depth constraint. Gray lines show the $95 \%$ confidence limits of estimated optimal flow.

properly managed situations (see Land Cover Codes for the 1998-2004 Cross-Sections).

3. Roost Macroforms. There was discussion during the 2001 workshop (Farmer and others, 2001) that sandbar configuration may affect the attractiveness of a roost site. Those who have conducted Whooping Crane aerial surveys have noticed that roost sites are clearly visible as relatively large, inundated "macroforms." These macroforms are clearly the result of sediment dynamics in the Platte River, but it is possible that Whooping Cranes are selecting roost sites based on some river-landscape characteristics, for example macroforms above a certain minimum size or breadth. 2-D hydraulic modeling might be useful for evaluating the macroforms available to, and used by Whooping Cranes.

4. Channel Degradation Due to Sediment Losses. One assumption associated with use of the roosting habitat models is that channel morphology within the central Platte River basin remains in dynamic equilibrium (Ziewitz, 1992). This assumption has been violated to some degree because many of the IFG4 data files were collected in the mid 1980's, and there have been measurable channel changes since that time; for example, degradations have been caused by the clear water returns from the J-2 hydropower plant. Recent information suggests that upstream portions of the central Platte River may not be in dynamic equilibrium as demonstrated by changes in mean elevation of the river channel at seven study sites (Murphy and others, 2004). Hence, the Service and Reclamation should continue putting a priority on re-measuring the cross sections at all study sites to characterize current conditions.

5. Proximity of Wet Meadows to Roost Sites. Our largescale, river segment evaluation of the C4R model showed that cranes tend to select roost sites in wider

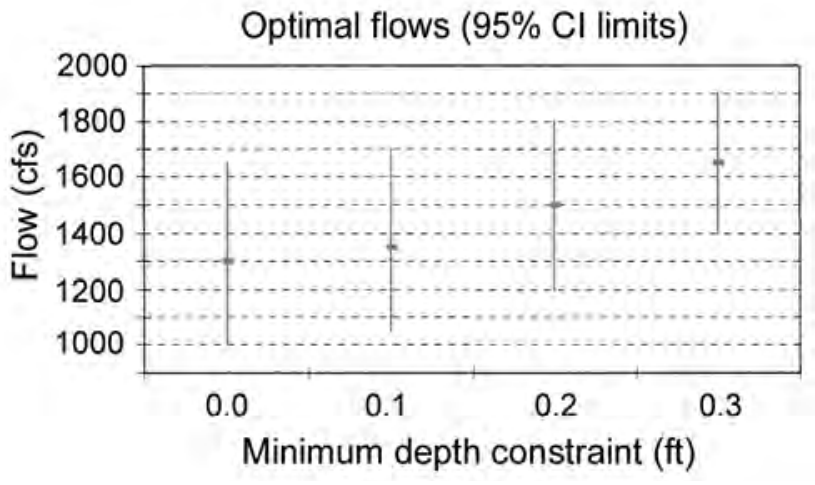

Figure 30. Variation in estimated optimal flow as a consequence of different assumptions about the minimum depth constraint. Gray lines show the $95 \%$ confidence limits of estimated optimal flow.

channels, but factors other than channel width per se may also be important. The river segments with the highest number of roost observations $(6,8 \mathrm{~B}, 9 \mathrm{BE}$, and 9BW) also have a relatively high amount of wet meadows in close proximity to potential roost channels. Whooping Crane biologists, including two that reviewed the depth criteria settings, emphasized the importance of wet meadow habitat. It would be important to develop a better understanding of how wet meadows affect roost suitability at a landscape scale.

6. Improving Disturbance Buffers. The original Whooping Crane suitability workshop in 1987 identified distance from a disturbance as an important variable. Disturbances were identified as any road, railway, bridge, or power line. The reported disturbance buffers ranged from a quarter mile (Carlson and others, 1990) to half a mile (Wingfield, 1993). Applications of the C4R model have only incorporated bridges as disturbances (Appendix A), but future applications of the model should incorporate all disturbances.

\section{Landscape Conditions Outside of the Central Platte} River. We evaluated the Whooping Crane model at two spatial scales within the river channel. However, Whooping Cranes may be influenced in their decisions to use the Platte River by conditions that occur within a much larger regional landscape. Richert (1999) identified three regions within Nebraska with the highest densities of night roosts. The three regions were the central Platte River, the western Rainwater Basin, and the Central Table Playa, all within 80 miles of each other. Understanding the habitat dynamics within these regions would give us a better understanding of the interactions that influence Whooping Crane usage from year to year.

8. Alternative Optimization Criteria. When it has been applied to set flow targets for the Platte River, the 
roosting habitat model has been run in the mode of identifying the "optimal" flow, defined as the flow that would maximize WUA within the entire study area. There may be alternative definitions of optimal that would be more meaningful. For example, it may be more meaningful to recommend a flow regime that results in some minimum amount of habitat (WUA) in each of the river segments. Or it may be more effective to maximize the WUA in segments that historically receive the highest Whooping Crane usage. There are no doubt other operational definitions of optimality that might be considered in further planning on the Platte River.

9. The hydraulic data need to be reviewed and updated in several ways:

a. The IFG4 data should be updated for every site; the most recent data from some sites is from the 1980's.

b. The spatial locations of segments and their associated study sites should be reviewed given the morphological changes that have occurred in the river since these units were originally defined, and that will occur in the future due to management. In particular, there is no study site to represent the river reaches where channel clearing is targeted. These areas should probably be defined as a new "segment" with a corresponding study site, cross-sections, and hydraulic data.

c. The linear regression equations that describe the splitting of river flow among segments (Hydrologic Reference Points, page 3) may no longer be accurate, and may need to be replaced with more realistic nonlinear relationships that more accurately describe flow splitting across a wide range of discharges.

\section{Protocols for Collecting Whooping Crane Observation Data}

During the course of our work we have been asked to conduct many evaluations using the single source of data available to us - the 71 Whooping Crane roost observations. Many of the analyses that we were asked to do, and in fact the analyses that we actually conducted probably stretched those data to their limits. There are some potentially useful analyses that simply cannot be conducted with the historic crane data. For example, during the February 2001 workshop and afterward we deliberated on how one might use the existing roost data to evaluate landscape factors that might be important in roost site selection. But it seems to us that the data are not sufficient for that purpose.

In the coming years there will be renewed efforts by the Whooping Crane Trust and the Service to survey Whooping Crane Roost sites along the river, and the February 2001 work- shop focused on defining protocols for future data collection that would help understand the landscape context of suitable roost sites. The recommended Whooping Crane survey protocol is as follows (a copy of the Service's previous data collection form for recording Whooping Crane observations is in Appendix D):

1. From an aerial survey, if a Whooping Crane is spotted in the river, acquire an aerial photo of the roost site with sufficient detail to delineate submerged bars and deeper channels. (It would also be desirable to photograph the high-use sites, to analyze the changes in bars through time).

2. Record discharge at the time of observation, as in the past.

3. Identify with the highest possible resolution, the specific coordinates of the crane's location in the river, especially with respect to the particular bar used for roosting.

4. Run three cross sections across the roost bar to characterize the bar in two dimensions, i.e., its width and size. Measure depths and identify obstructions as in the past, and also measure unobstructed channel width.

5. Also collect random cross section data in the same segment for later analysis to determine if there is selection for particular sandbar traits.

6. Measure distances to the following landscape features:

a. nearest wet meadow, and

b. nearest obstruction (however obstructions are defined - see previous section).

7. Identify if the Whooping Crane is associated with Sandhill Cranes or not.

\section{Acknowledgments}

The following individuals participated in workshops, provided data and shared insights about Whooping Crane ecology, and/or reviewed earlier versions of this report: D. Anderson, U.S. Fish and Wildlife Service. M. Armbruster, U.S. Bureau of Reclamation; J. Austin, U.S. Geological Survey, Northern Prairie Wildlife Research Center; K. Bovee, U.S. Geological Survey; D. Carlson, U.S. Fish and Wildlife Service; C. Davis, Oklahoma State University; J. Dinan, Nebraska Game and Parks Commission; L. Fotherby, U.S. Bureau of Reclamation; J. Jenniges, Nebraska Public Power District; B. Johns, Canadian Wildlife Service; D. Johnson, U.S. Geological Survey; P. Kinzel, U.S. Geological Survey; J. Lewis, U.S. Fish and Wildlife Service (retired); G. Lingle, University of Nebraska; P. Mielke, Colorado State University, 
R. Milhous, U.S. Geological Survey; and F. Chavez-Ramirez, Platte River Whooping Crane Management Trust. The U.S. Fish and Wildlife Service, Denver, Colorado and the U.S. Geological Survey provided financial support for the project.

\section{References Cited}

Anonymous, 1992, Summary of Instream Flow Incremental Methodology Whooping Crane roosting habitat models, report attached to February 7, 1992 memo from USFWS Grand Island Office to Greg Winfield, Nebraska Game and Parks Commission.

Austin, J.E., and Richert, A.L., 2001, A comprehensive review of observational and site evaluation data of migrant Whooping Cranes in the United States, 1943-1999: U.S. Geological Survey, Northern Prairie Wildlife Research Center, Jamestown, ND, 157 p.

Bovee, K.D., 1982, A guide to stream habitat analysis using the Instream Flow Incremental Methodology: Information Paper No. 12, FWS/OBS-82/26, 242 p.

Bovee, K.D., and Milhous, R., 1978, Hydraulic simulation in instream flow studies: Theory and techniques: Instream Flow Information Paper 5, U.S. Fish and Wildlife Service, Cooperative Instream Flow Service Group, Ft. Collins, Colorado.

Cade, B.S., Terrell, J.W., and Schroeder, R.L., 1999, Estimating effects of limiting factors with regression quantiles: Ecology, v. 80, p. 311-323.

Carlson, D., 1994, Supplemental information on the Whooping Crane roost habitat model: computation of the depth SI: A report from the Whooping Crane modeling subcommittee to the Biology Work Group, Platte River Management Joint Study, January 25, 1994.

Carlson, D., Holz, D., Woodward, D., and Ziewitz, J., 1990, Whooping Crane roosting habitat simulation model for the Platte River in Nebraska: Report to the Biology Workgroup, Platte River Management Joint Study.

Faanes, C.A., Johnson, D.H., and Lingle, G.R., 1992, Characteristics of Whooping Crane sites in the Platte River, in Proceedings of the 1988 North American Crane Workshop, International Crane Foundation, Baraboo, Wisconsin, p. 90-94.

Farmer, A.H., Cade, B.S., Terrell, J.W., Henriksen, J.H., Schneider, D.M., and Fancher, T.S., 2000, Evaluation of a suitability model and associated procedures utilized for assessment of Whooping Crane (Grus americana) habitat in the Platte River, Nebraska: A report to the U.S. Fish and Wildlife Service, Region 6, Contract 1448-60181-99-N292, Midcontinent Ecological Science Center, Fort Collins, Colorado.

Farmer, A.H., Cade, B.S., Terrell, J.W., and Henriksen, J.H., 2001, Results of a Whooping Crane roosting habitat model workshop: A report to the U.S. Fish and Wildlife Service, Region 6, Contract 1448-60181-99-N292, Midcontinent Ecological Science Center, Fort Collins, Colorado.

General Reporting Service. Inc., 1997, In the matter of applications A-17329 through A-17333 for permits to appropriate water for instream flows on the Plate River, Water Division 1-A, 2-A \& 2-B: Transcripts of February 20, 1997 hearing before the Nebraska State Department of Water Resources, J.M. Hess, Director and hearing officer.

Mielke, P.W., and Berry, K.J., 2001, Permutation methods: A distance function approach, Springer-Verlag, $352 \mathrm{p}$.

Milhous, R. T., Wegner, D.L., and Waddle, T., 1984, Users guide to the Physical Habitat Simulation System: Instream Flow Information Paper 11, U.S. Fish and Wildlife Service, FWS/OBS-81/43 Revised.

Murphy, P.J., Randle, T.J., Fotherby, L.M., and Dario, J.A., 2004, Platte River Channel: History and restoration: U.S. Bureau of Reclamation, Denver, Colorado, 149 p.

National Research Council of the National Academies, 2004, Endangered and threatened species of the Platte River, Interim Report: The National Academies Press, Washington, D.C.

Richert, A.L.D., 1999, Multiple scale analyses of Whooping Crane habitat in Nebraska: Ph.D. dissertation, University of Nebraska, Lincoln, 175 p.

Rodney, M., 2002, Calibration and validation of the Central Platte OPSTUDY model: Ph.D. dissertation, University of Nebraska, Lincoln, 175 p.

Shenk, T.M., and Armbruster, M.J., 1986, Whooping Crane habitat criteria for the Big Bend area of the Platte River: U.S. Fish and Wildlife Service, 34 p. + appendices.

U.S. Bureau of Reclamation, 1987, Platte River instream flow incremental methodology hydraulic simulation result: Prairie Bend and Platte River Joint Management Study, Nebraska-Kansas Projects Office of the U.S. Bureau of Reclamation, February 1987.

U.S. Bureau of Reclamation, 1989, Prairie Bend unit, P-S MBP, Nebraska, Regional Directors planning report/draft environmental statement: Hydrology appendix, vol. 1 of 3. 
U.S. Fish and Wildlife Service, 1987, Whooping Crane roosting habitat criteria for the Platte and North Platte Rivers in Nebraska: U.S. Fish and Wildlife Service, Grand Island, Nebraska, February 1987.

U.S. Geological Survey, 2000, Whooping Crane roosting habitat study series (Maps a through i). U.S. Geological Survey, Midcontinent Ecological Science Center, GIS and Remote Sensing Project.

Wilkinson, L., 1999, SYSTAT, Version 8.0: SPSS Incorporated, Chicago, Illinois.

Williamson, S., 1983, Platte River database documentation: Rapid Assessment Methods Group, Western Energy and Land Use Team (WELUT), U.S. Fish and Wildlife Service, Fort Collins, Colorado.
Wingfield, G.A., 1993, Request for instream flow appropriation for the Central Platte River, Flows to maintain Whooping Crane roosting habitat: Nebraska Game and Parks Commission, Lincoln.

Ziewitz, J.W., 1987, Whooping Crane riverine roost habitat suitability model program documentation: Platte River Whooping Crane Maintenance Trust, Inc., Grand Island, Nebraska.

Ziewitz, J.W., 1992, Whooping Crane riverine roosting habitat suitability model, in Proceedings of the 1988 North American Crane Workshop: State of Florida Game and Freshwater Fish Commission, p. 71-81. 
Appendix A. Study Area Maps 


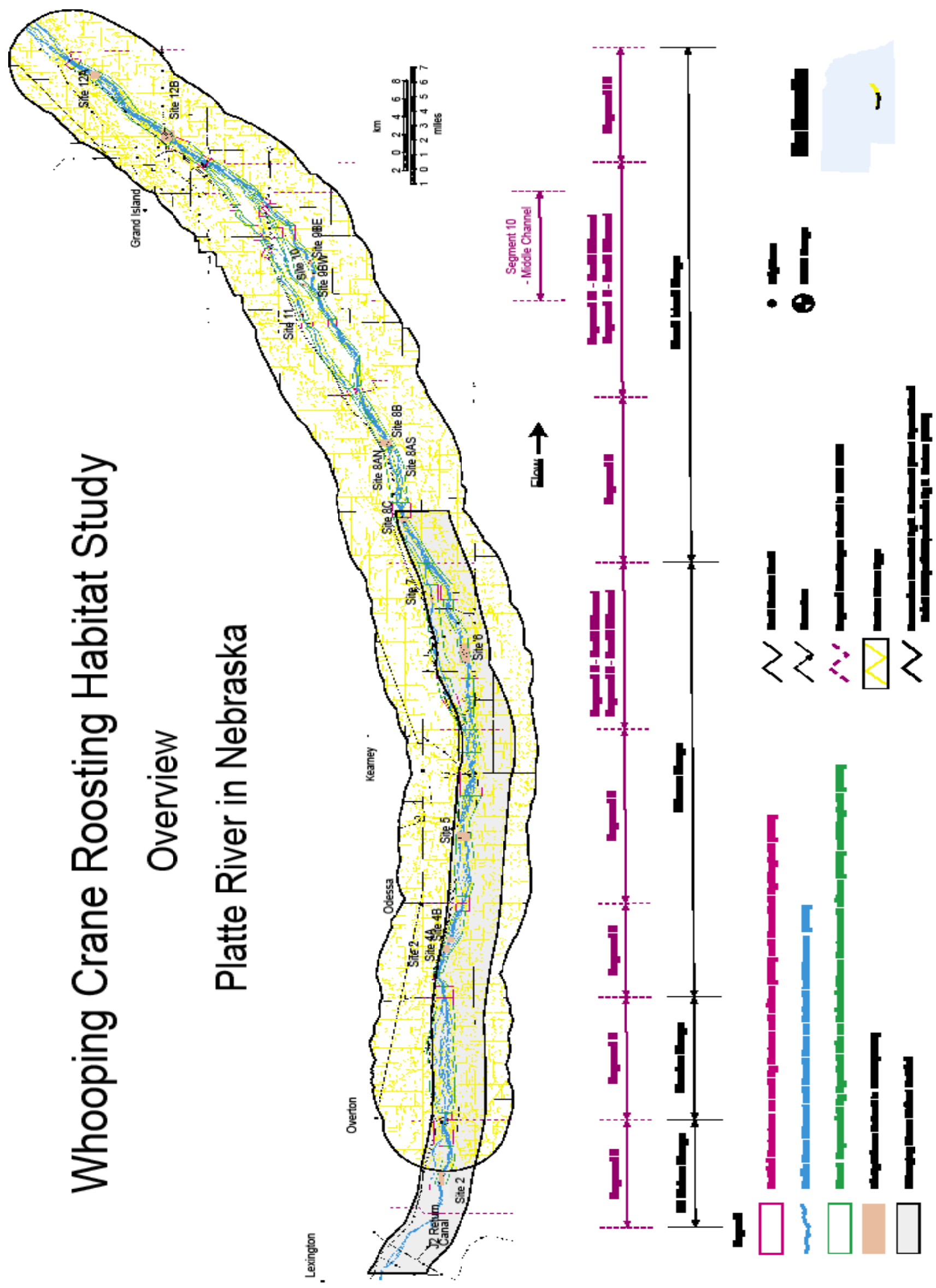



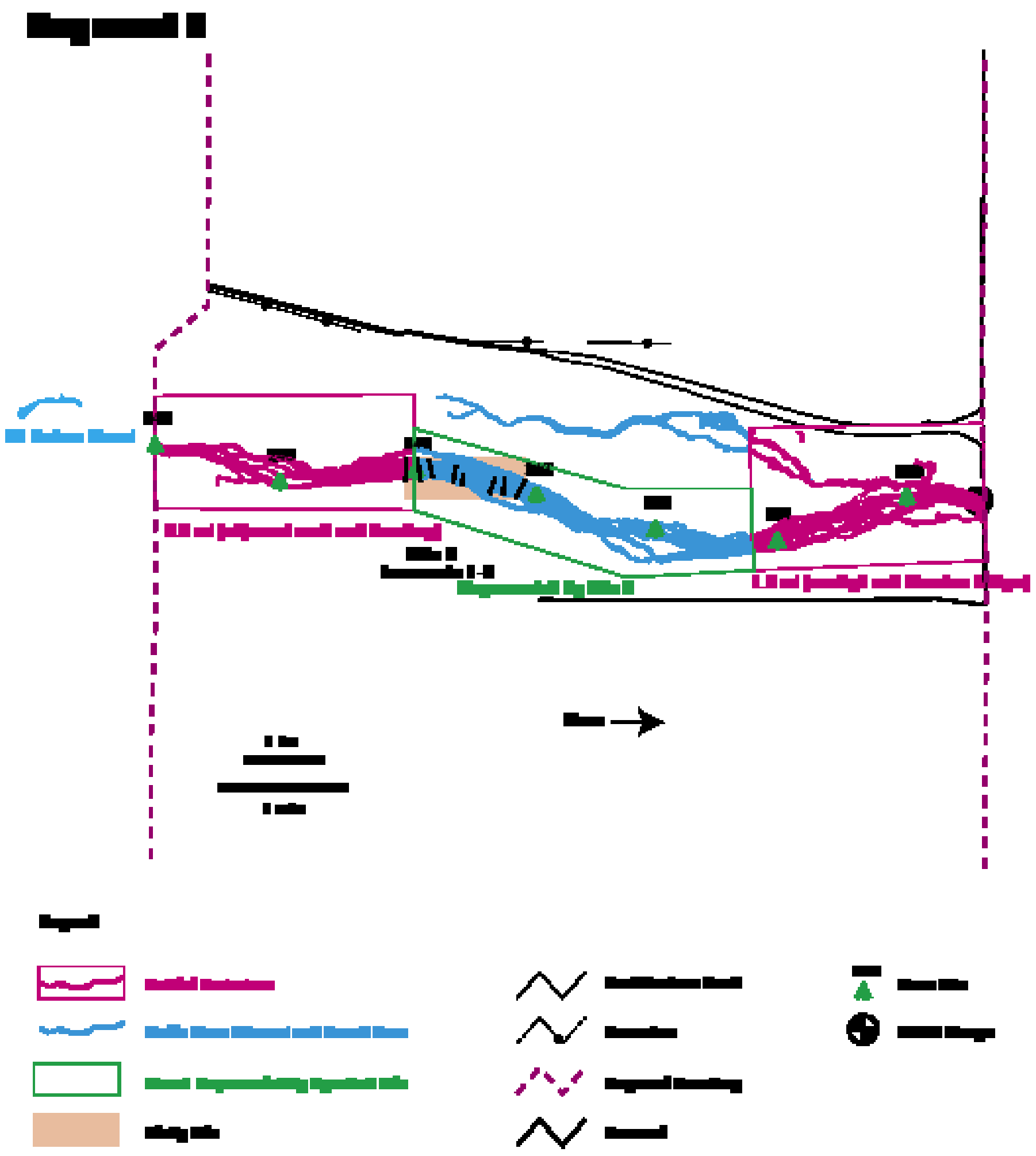

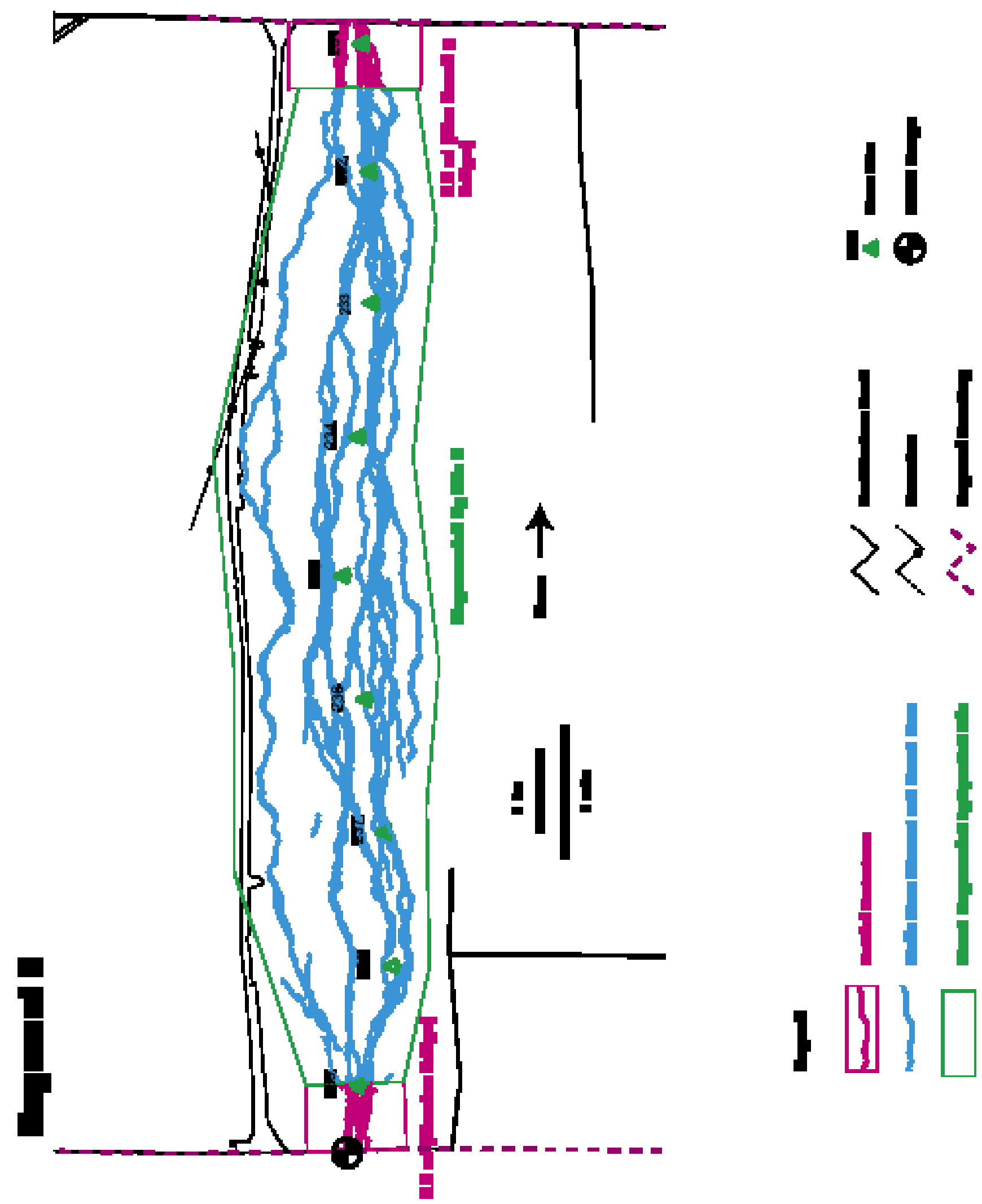


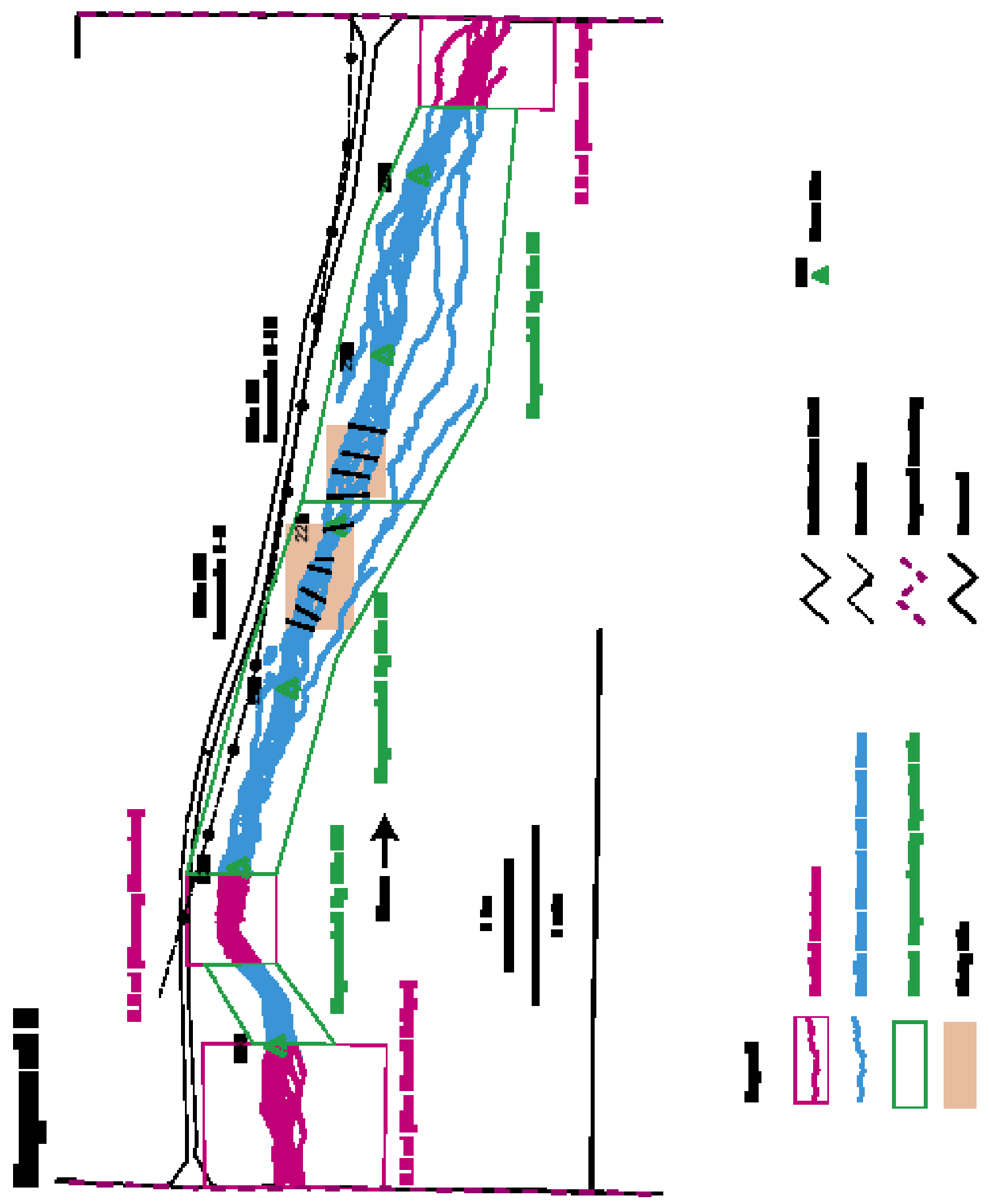




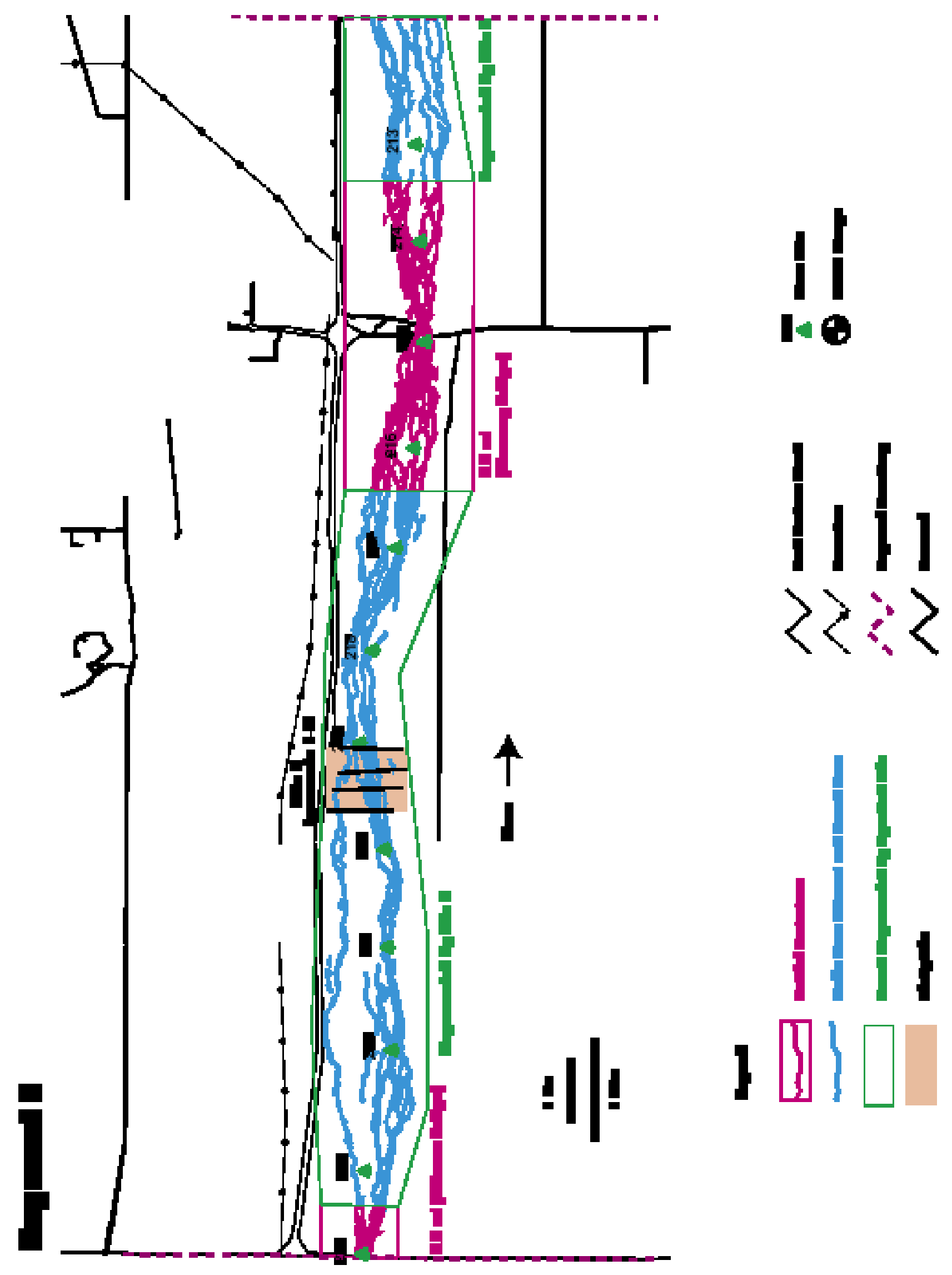




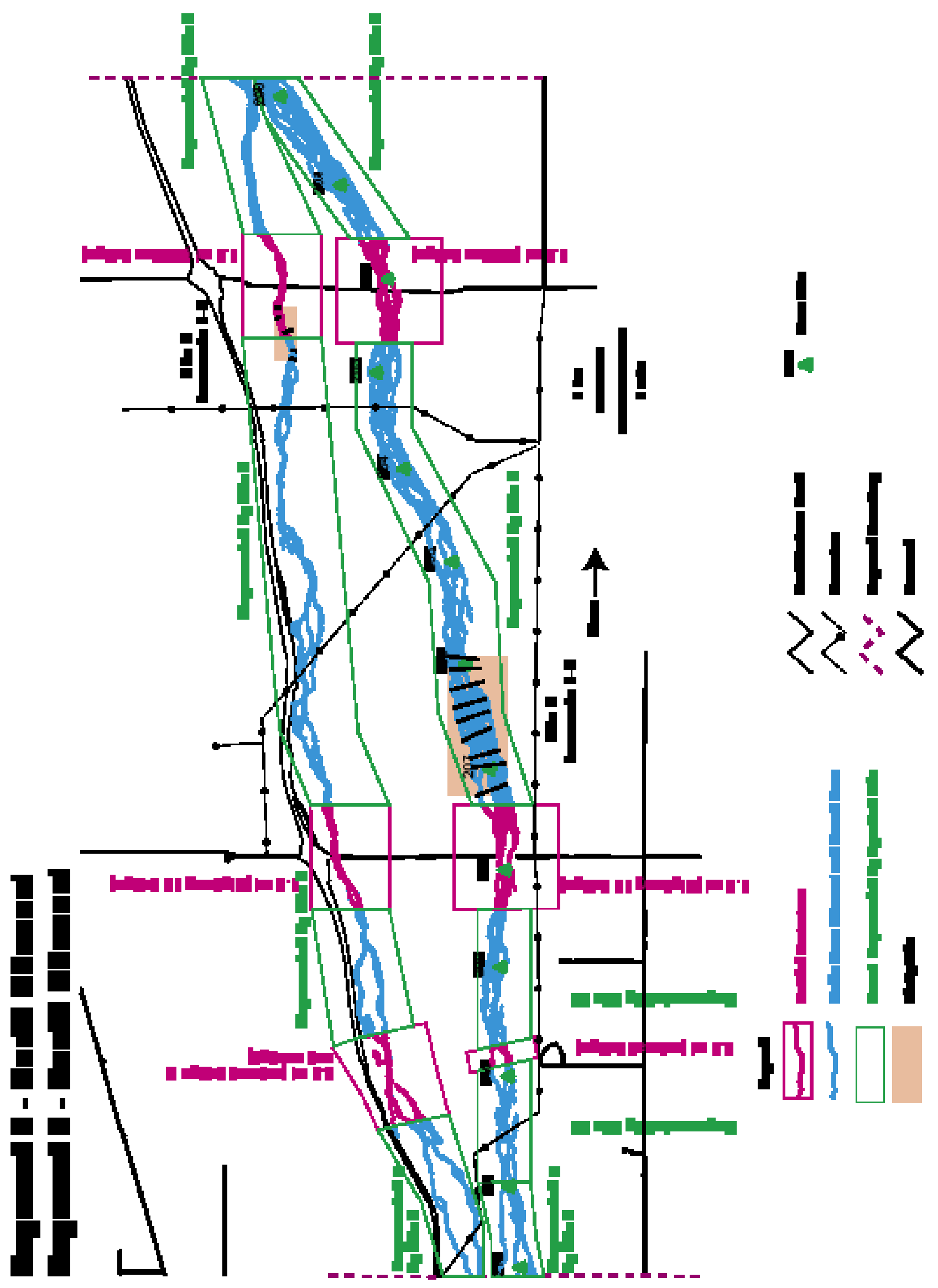




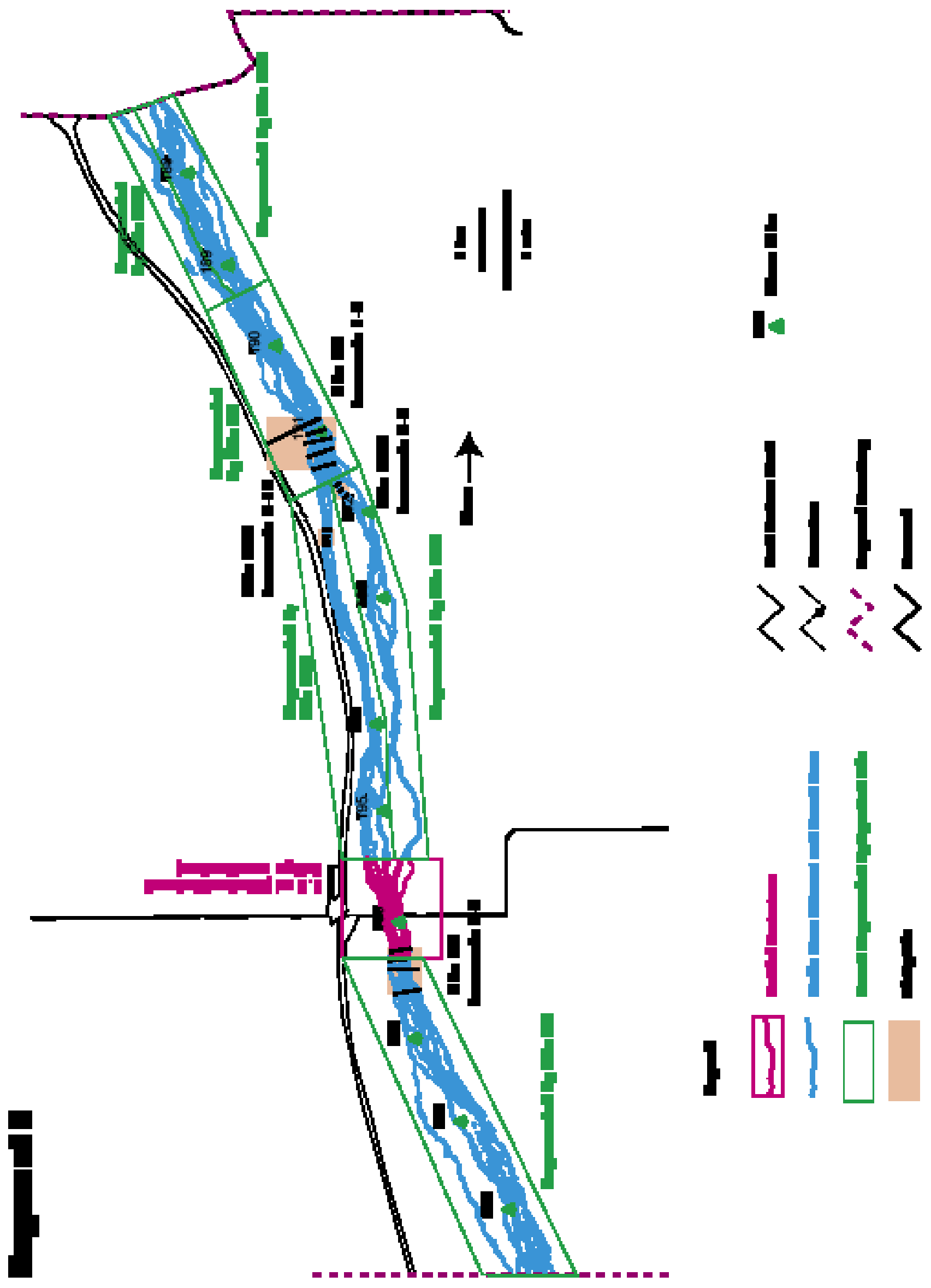




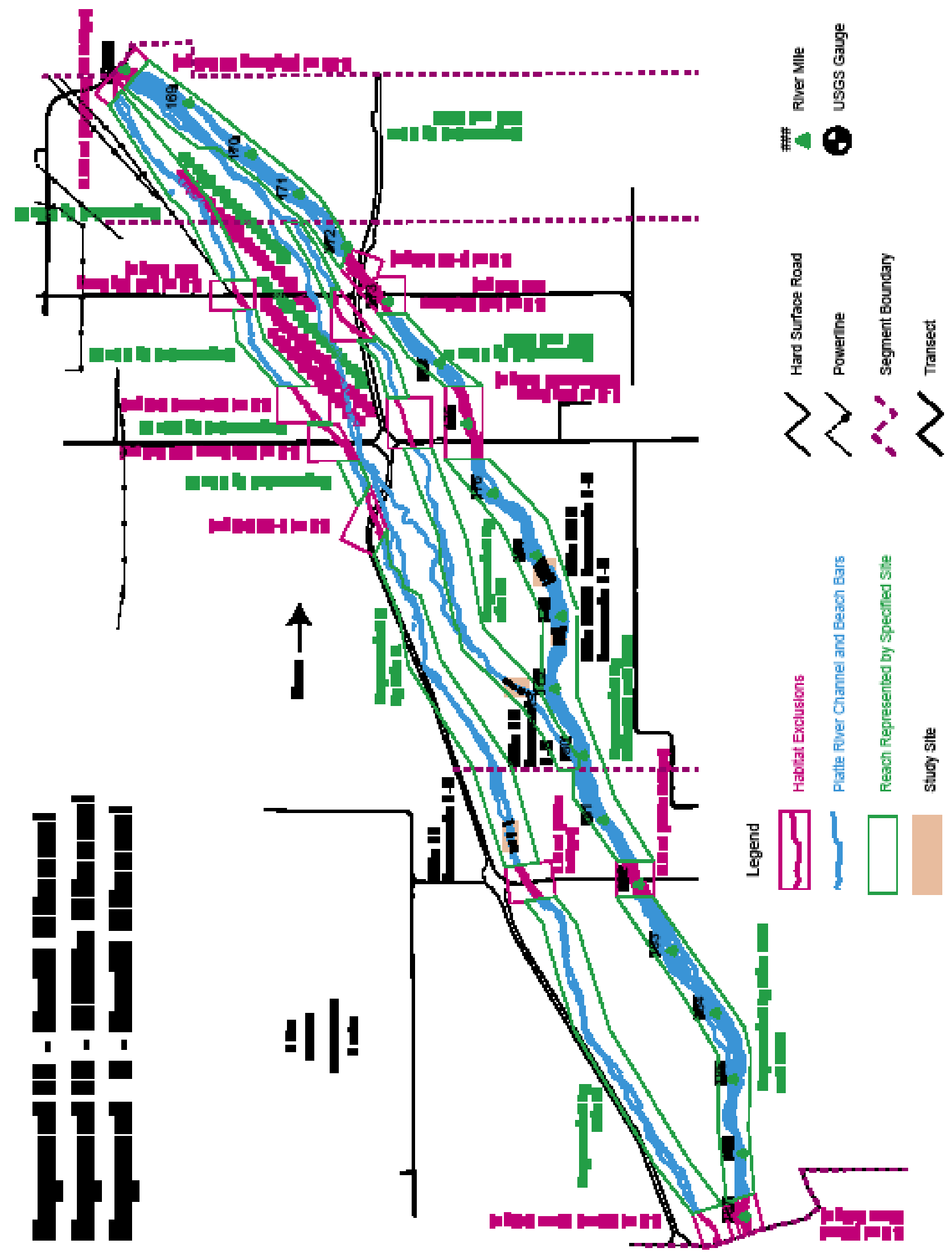




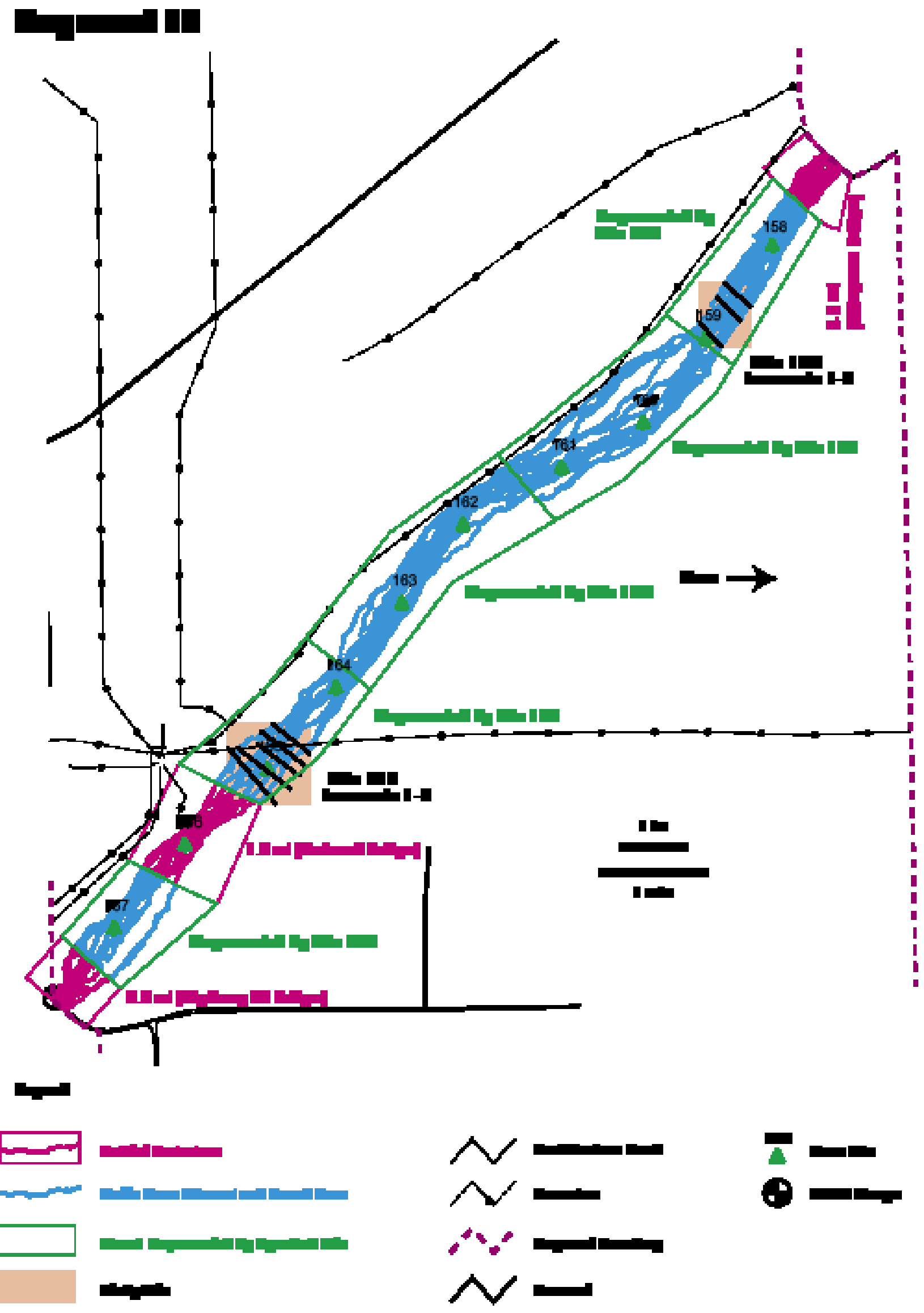




\section{Appendix B. Segment Lengths and Excluded Areas}

In a braided channel such as the Platte River, determining the channel length is problematic. Typical approaches used in a single channel, such as following the channel centerline, were not practical because of numerous crossovers and islands within some segments. Instead, we calculated segment length as the mean of the length of the north bank and the length of the south bank of the selected segment (either the main channel or a distinct secondary channel). Bank length was measured along the edge of the water (or beach bar) except that when an intrusion had a length that exceeded the width of its base, the measurement followed the base of the intrusion. Various sources of human disturbance (e.g., bridges) also affect WUA by excluding areas of habitat that might otherwise be usable by cranes. Lengths of exclusions were calculated in a similar manner. The boundaries of exclusion were set by rule (e.g., 0.5 mile straight-line distance either side of a bridge as shown in Appendix A). Then the actual exclusion length was determined using the same measuring convention as for segment length. 


\section{Appendix B. Segment lengths and excluded areas.-Continued.}

\begin{tabular}{|c|c|c|}
\hline Location & Length (feet) & Length (miles) \\
\hline Segment 2 (J-2 to Overton Bridge) & $38,536.94$ & 7.30 \\
\hline Overton Bridge exclusion & $11,060.84$ & 2.09 \\
\hline Adjacent road and dwelling exclusion & $11,217.08$ & 2.12 \\
\hline Length after exclusions (represented by site 2 ) & $15,163.91$ & 2.87 \\
\hline Segment 3 (Overton Bridge to Elm Creek Bridge) & $49,613.00$ & 9.40 \\
\hline Elm Creek Bridge exclusion & $2,868.75$ & 0.54 \\
\hline Overton Bridge exclusion & $2,980.15$ & 0.56 \\
\hline Length after exclusions (represented by site 5) & $43,764.10$ & 8.29 \\
\hline Segments 4A, 4B (Elm Creek Bridge to Odessa Bridge) & $38,732.32$ & 7.34 \\
\hline Elm Creek Bridge exclusion & $4,474.08$ & 0.85 \\
\hline Kearney Diversion exclusion & $3,150.97$ & 0.60 \\
\hline Odessa Bridge exclusion & $3,069.41$ & 0.58 \\
\hline Length after exclusions (represented by Site $4 \mathrm{~A}$ ) & $15,100.66$ & 2.86 \\
\hline Length after exclusions (represented by Site 4B) & $12,937.20$ & 2.45 \\
\hline $\begin{array}{l}\text { Segment } 5 \text { (Odessa Bridge to Fort Farm Island ( } 3.0 \text { miles below Kearney } \\
\text { Bridge) }\end{array}$ & $69,521.56$ & 13.17 \\
\hline Kearney Bridge exclusion & $17,698.23$ & 3.35 \\
\hline Odessa Bridge exclusion & $2,836.74$ & 0.54 \\
\hline Length after exclusions (represented by Site 5) & $48,986.60$ & 9.28 \\
\hline Segment 6 (South channel, length of Fort Farm Island) & $68,998.96$ & 13.07 \\
\hline Railroad Bridge exclusion & $1,130.11$ & 0.21 \\
\hline Highway 10 Bridge exclusion & $5,908.00$ & 1.12 \\
\hline Gibbon Bridge exclusion & $6,275.48$ & 1.19 \\
\hline Length after exclusions (represented by Site 6) & $43,013.37$ & 8.15 \\
\hline Length after exclusions (represented by Site 4B) & $12,672.00$ & 2.40 \\
\hline \multicolumn{3}{|l|}{ Potential Powerline Exclusions } \\
\hline Length of 1st Powerline & $3,292.39$ & 0.62 \\
\hline Length of 2nd Powerline & $3,242.29$ & 0.61 \\
\hline Length of 3rd Powerline & $2,812.86$ & 0.53 \\
\hline Segment 7, North Channel, length of Fort Farm Island & 68619.48 & 13.00 \\
\hline Railroad Bridge exclusion & $5,426.98$ & 1.03 \\
\hline Highway 10 Bridge exclusion & $5,769.85$ & 1.09 \\
\hline Gibbon Bridge exclusion & $5,930.21$ & 1.12 \\
\hline Length after exclusions (represented by Site 7) & 51492.44 & 9.75 \\
\hline \multicolumn{3}{|l|}{ Potential Powerline Exclusions } \\
\hline Length of 1st Powerline & $3,417.79$ & 0.65 \\
\hline Length of 2nd Powerline & $5,360.96$ & 1.02 \\
\hline Length of 3rd Powerline & $2,795.42$ & 0.53 \\
\hline
\end{tabular}




\section{Appendix B. Segment lengths and excluded areas.-Continued.}

\begin{tabular}{|c|c|c|}
\hline Location & Length (feet) & Length (miles) \\
\hline Segments 8An, 8AS, 8B, 8C (Fort Farm Island to Shoemaker Island) & $105,022.88$ & 19.89 \\
\hline Shelton/RR Bridge exclusion & $5,790.21$ & 1.10 \\
\hline Segment 8AN & $32,941.61$ & 6.24 \\
\hline Segment 8AS & $34,773.02$ & 6.59 \\
\hline Segment 8B & $11,790.55$ & 2.23 \\
\hline Segment $8 \mathrm{C}$ & $19,727.48$ & 3.74 \\
\hline Length of segment 8AN (represented by Site 8AN) & $32,941.61$ & 6.24 \\
\hline Length of segment 8AS (represented by Site 8AS) & $34,773.02$ & 6.59 \\
\hline Length of segment 8B (represented by Site 8B) & $11,790.55$ & 2.23 \\
\hline Length of segment $8 \mathrm{C}$ (represented by Site $8 \mathrm{C}$ ) & $19,727.48$ & 3.74 \\
\hline Segments 9BE, 9BW, South channel, Shoemaker and Mormon Islands & $107,064.39$ & 20.28 \\
\hline Alda Bridge exclusion & $3,050.28$ & 0.58 \\
\hline Highway 281/Railroad Bridge exclusion & $5,686.10$ & 1.08 \\
\hline Highway 34 Bridge exclusion & $1,541.32$ & 0.29 \\
\hline I-80 Bridge exclusion & $2,868.45$ & 0.54 \\
\hline Wood River Bridge exclusion & $2,888.20$ & 0.55 \\
\hline Old Highway 281 Bridge exclusion & $2,755.80$ & 0.52 \\
\hline Length of Segment 9BE (represented by Sites 9BE) & $44,137.11$ & 8.36 \\
\hline Length of Segment 9BW (represented by Sites 9BW) & $44,137.11$ & 8.36 \\
\hline Segment 10, Middle channel, Mormon Island & $48,078.38$ & 9.11 \\
\hline Highway 281/Railroad Bridge exclusion & $4,087.50$ & 0.77 \\
\hline I-80/old Highway 281 Bridge exclusion & $5,084.17$ & 0.96 \\
\hline Length after exclusions (represented by Site 10) & $38,906.71$ & 7.37 \\
\hline Segment 11 (Middle channel, Shoemaker Island to Highway 34 Bridge) & $102,905.10$ & 19.49 \\
\hline Alda Bridge Exclusion & $2,995.99$ & 0.57 \\
\hline Highway 281 Bridge exclusion & $3,907.26$ & 0.74 \\
\hline Highway 34 Bridge exclusion & $1,904.01$ & 0.36 \\
\hline I-80 Bridge exclusion & $3,766.86$ & 0.71 \\
\hline Railroad Bridge exclusion & $3,580.19$ & 0.68 \\
\hline Wood River Bridge exclusion & $3,008.94$ & 0.57 \\
\hline Old Highway 281 Bridge exclusion & 2,564.01 & 0.49 \\
\hline Length after exclusions (represented by Site 11) & $81,177.85$ & 15.37 \\
\hline Segment 12 (Highway 34 Bridge to Chapman Bridge) & $60,030.23$ & 11.37 \\
\hline Chapman Bridge exclusion & $2,903.30$ & 0.55 \\
\hline Highway 34 Bridge exclusion & $3,076.86$ & 0.58 \\
\hline Railroad Bridge exclusion & $5,932.54$ & 1.12 \\
\hline Length of Segment 12A (represented by Site $12 \mathrm{~A}$ ) & $27,404.48$ & 5.19 \\
\hline Length of Segment 12B (represented by Site 12B) & $20,713.05$ & 3.92 \\
\hline Potential Powerline Exclusion & $3,037.86$ & 0.58 \\
\hline
\end{tabular}


Appendix C. Table of UTM Coordinates for Study Site Cross Sections 


\section{Appendix C. Table of UTM Coordinates for Study Site Cross Sections}

\begin{tabular}{|c|c|c|c|c|c|c|c|}
\hline Study site & Cross section & X-coordinate & Y-coordinate & Study site & Cross section & X-coordinate & Y-coordinate \\
\hline 2 & 1 & $448,659.80$ & 4503698 & 6 & 3 & $506,724.10$ & 4501696 \\
\hline 2 & 1 & $448,540.50$ & 4503468 & 6 & 3 & $506,851.30$ & 4501176 \\
\hline 2 & 2 & $448,399.00$ & 4503718 & 6 & 4 & $506,447.00$ & 4501643 \\
\hline 2 & 2 & $448,384.30$ & 4503501 & 6 & 4 & $506,594.40$ & 4501132 \\
\hline 2 & 3 & $448,283.30$ & 4503715 & 6 & 5 & $506,225.30$ & 4501604 \\
\hline 2 & 3 & $448,211.60$ & 4503519 & 6 & 5 & $506,398.00$ & 4501097 \\
\hline 2 & 4 & $447,889.50$ & 4503765 & 6 & 6 & $505,936.50$ & 4501519 \\
\hline 2 & 4 & $447,866.30$ & 4503634 & 6 & 6 & $506,150.20$ & 4501057 \\
\hline 2 & 5 & $447,798.20$ & 4503873 & 6 & 7 & $505,668.30$ & 4501394 \\
\hline 2 & 5 & $447,762.30$ & 4503649 & 6 & 7 & $505,795.90$ & 4500925 \\
\hline 2 & 6 & $447,458.40$ & 4503948 & 6 & 8 & $505,531.50$ & 4501356 \\
\hline 2 & 6 & $447,508.40$ & 4503720 & 6 & 8 & $505,591.30$ & 4500851 \\
\hline 2 & 7 & $447,317.40$ & 4503978 & 6 & 9 & $505,093.00$ & 4501294 \\
\hline 2 & 7 & $447,354.40$ & 4503679 & 6 & 9 & $505,275.60$ & 4500812 \\
\hline 2 & 8 & $447,179.40$ & 4503976 & 7 & 1 & $512,620.90$ & 4504402 \\
\hline 2 & 8 & $447,167.40$ & 4503676 & 7 & 1 & $512,621.10$ & 4504309 \\
\hline $4 \mathrm{~A}$ & 1 & $473,807.40$ & 4503052 & 7 & 2 & $512,446.90$ & 4504390 \\
\hline $4 \mathrm{~A}$ & 1 & $473,862.30$ & 4502781 & 7 & 2 & $512,468.60$ & 4504322 \\
\hline $4 \mathrm{~A}$ & 2 & $473,557.10$ & 4503127 & 7 & 3 & $512,224.90$ & 4504272 \\
\hline $4 \mathrm{~A}$ & 2 & $473,548.30$ & 4502963 & 7 & 3 & $512,278.80$ & 4504134 \\
\hline $4 \mathrm{~A}$ & 3 & $473,482.30$ & 4503194 & 7 & 4 & $511,964.90$ & 4504151 \\
\hline $4 \mathrm{~A}$ & 3 & $473,431.10$ & 4502976 & 7 & 4 & $511,950.80$ & 4504079 \\
\hline $4 \mathrm{~A}$ & 4 & $473,288.10$ & 4503252 & 7 & 5 & $511,843.80$ & 4504172 \\
\hline $4 \mathrm{~A}$ & 4 & $473,170.70$ & 4503034 & 7 & 5 & $511,806.20$ & 4504078 \\
\hline $4 \mathrm{~A}$ & 5 & $473,120.00$ & 4503294 & $8 \mathrm{AN}$ & 9 & $528,130.10$ & 4508115 \\
\hline $4 \mathrm{~A}$ & 5 & $473,007.70$ & 4503094 & $8 \mathrm{AN}$ & 9 & $528,132.60$ & 4507986 \\
\hline $4 \mathrm{~A}$ & 6 & $472,998.00$ & 4503384 & $8 \mathrm{AN}$ & 10 & $528,030.70$ & 4508113 \\
\hline $4 \mathrm{~A}$ & 6 & $472,922.20$ & 4503134 & $8 \mathrm{AN}$ & 10 & $528,028.50$ & 4508003 \\
\hline $4 B$ & 7 & $474,748.60$ & 4502819 & $8 \mathrm{AN}$ & 11 & $527,989.60$ & 4508119 \\
\hline 4B & 7 & $474,686.70$ & 4502496 & $8 \mathrm{AN}$ & 11 & $527,943.40$ & 4508002 \\
\hline 4B & 8 & $474,504.30$ & 4502881 & $8 \mathrm{AN}$ & 12 & $527,900.20$ & 4508119 \\
\hline $4 B$ & 8 & $474,443.70$ & 4502567 & $8 \mathrm{AN}$ & 12 & $527,897.60$ & 4508002 \\
\hline $4 \mathrm{~B}$ & 9 & $474,338.30$ & 4502962 & 8AS & 6 & $528,835.40$ & 4507918 \\
\hline $4 B$ & 9 & $474,288.50$ & 4502639 & $8 \mathrm{AS}$ & 6 & $528,896.40$ & 4507843 \\
\hline $4 B$ & 10 & $474,104.50$ & 4503008 & $8 \mathrm{AS}$ & 7 & $528,738.10$ & 4507860 \\
\hline 4B & 10 & $474,124.90$ & 4502648 & $8 \mathrm{AS}$ & 7 & $528,796.40$ & 4507808 \\
\hline 5 & 1 & $486,320.80$ & 4501888 & 8AS & 8 & $528,640.60$ & 4507787 \\
\hline 5 & 1 & $486,287.80$ & 4500899 & 8AS & 8 & $528,701.60$ & 4507709 \\
\hline 5 & 2 & $485,922.80$ & 4501888 & $8 \mathrm{~B}$ & 1 & $529,513.00$ & 4509019 \\
\hline 5 & 2 & $485,986.80$ & 4500849 & $8 \mathrm{~B}$ & 1 & $529,927.60$ & 4508161 \\
\hline 5 & 3 & $485,660.80$ & 4501981 & $8 \mathrm{~B}$ & 2 & $529,660.70$ & 4508387 \\
\hline 5 & 3 & $485,693.80$ & 4500910 & $8 \mathrm{~B}$ & 2 & $529,752.10$ & 4508072 \\
\hline 5 & 4 & $485,331.80$ & 4502082 & $8 \mathrm{~B}$ & 3 & $529,519.10$ & 4508419 \\
\hline 5 & 4 & $485,341.80$ & 4501043 & $8 \mathrm{~B}$ & 3 & $529,610.70$ & 4507975 \\
\hline 6 & 1 & $507,242.20$ & 4501746 & $8 \mathrm{~B}$ & 4 & $529,300.80$ & 4508366 \\
\hline 6 & 1 & $507,194.10$ & 4501252 & $8 \mathrm{~B}$ & 4 & $529,446.60$ & 4507947 \\
\hline 6 & 2 & $507,039.60$ & 4501710 & $8 \mathrm{~B}$ & 5 & $529,107.10$ & 4508284 \\
\hline 6 & 2 & $507,058.30$ & 4501232 & $8 \mathrm{~B}$ & 5 & $529,203.20$ & 4507909 \\
\hline
\end{tabular}




\section{Appendix C. Table of UTM Coordinates for Study Site Cross Sections- Continued.}

\begin{tabular}{|c|c|c|c|c|c|c|c|}
\hline Study site & Cross section & X-coordinate & Y-coordinate & Study site & Cross section & X-coordinate & Y-coordiante \\
\hline $8 \mathrm{C}$ & 1 & $521,355.00$ & 4507035 & 10 & 3 & $547,175.90$ & 4515996 \\
\hline $8 \mathrm{C}$ & 1 & $521,413.70$ & 4506691 & 10 & 4 & $547,064.80$ & 4516008 \\
\hline $8 \mathrm{C}$ & 2 & $521,210.50$ & 4507058 & 10 & 4 & $547,127.60$ & 4515940 \\
\hline $8 \mathrm{C}$ & 2 & $521,259.10$ & 4506644 & 10 & 5 & $547,021.00$ & 4515957 \\
\hline $8 \mathrm{C}$ & 3 & $521,079.50$ & 4507046 & 10 & 5 & $547,084.40$ & 4515903 \\
\hline $8 \mathrm{C}$ & 3 & $521,077.90$ & 4506559 & 10 & 6 & $546,938.00$ & 4515841 \\
\hline $8 \mathrm{C}$ & 4 & $520,677.20$ & 4506970 & 10 & 6 & $547,033.80$ & 4515801 \\
\hline $8 \mathrm{C}$ & 4 & $520,749.50$ & 4506538 & 10 & 7 & $546,894.60$ & 4515772 \\
\hline $9 \mathrm{BE}$ & 1 & $549,680.40$ & 4515493 & 10 & 7 & $546,994.80$ & 4515719 \\
\hline $9 \mathrm{BE}$ & 1 & $549,877.60$ & 4515268 & 10 & 8 & $546,852.80$ & 4515647 \\
\hline $9 \mathrm{BE}$ & 2 & $549,644.40$ & 4515454 & 10 & 8 & $546,954.10$ & 4515605 \\
\hline $9 \mathrm{BE}$ & 2 & $549,812.50$ & 4515240 & 11 & 1 & $544,025.90$ & 4516177 \\
\hline $9 \mathrm{BE}$ & 3 & $549,603.30$ & 4515436 & 11 & 1 & $544,104.80$ & 4515956 \\
\hline $9 \mathrm{BE}$ & 3 & $549,750.40$ & 4515213 & 11 & 2 & $543,800.40$ & 4516092 \\
\hline $9 \mathrm{BE}$ & 4 & $549,555.60$ & 4515418 & 11 & 2 & $543,808.50$ & 4515903 \\
\hline $9 \mathrm{BE}$ & 4 & $549,675.40$ & 4515178 & 11 & 3 & $543,638.90$ & 4516056 \\
\hline $9 \mathrm{BE}$ & 5 & $549,491.90$ & 4515395 & 11 & 3 & $543,651.10$ & 4515879 \\
\hline 9BE & 5 & $549,628.40$ & 4515146 & 11 & 4 & $543,553.40$ & 4516024 \\
\hline $9 \mathrm{BE}$ & 6 & $549,408.60$ & 4515345 & 11 & 4 & $543,542.80$ & 4515869 \\
\hline $9 \mathrm{BE}$ & 6 & $549,553.80$ & 4515084 & 11 & 5 & $543,437.80$ & 4516006 \\
\hline $9 \mathrm{BE}$ & 7 & $549,300.50$ & 4515284 & 11 & 5 & $543,492.40$ & 4515853 \\
\hline $9 \mathrm{BE}$ & 7 & $549,449.50$ & 4515030 & $12 \mathrm{~A}$ & 1 & $570,433.30$ & 4535419 \\
\hline 9BW & 1 & $548,328.20$ & 4515127 & $12 \mathrm{~A}$ & 1 & $570,834.30$ & 4535061 \\
\hline 9BW & 1 & $548,340.20$ & 4514790 & $12 \mathrm{~A}$ & 2 & $570,324.70$ & 4535215 \\
\hline 9BW & 2 & $548,244.00$ & 4515091 & $12 \mathrm{~A}$ & 2 & $570,677.60$ & 4534849 \\
\hline 9BW & 2 & $548,240.00$ & 4514825 & $12 \mathrm{~A}$ & 3 & $570,063.80$ & 4534832 \\
\hline 9BW & 3 & $548,180.70$ & 4515089 & $12 \mathrm{~A}$ & 3 & $570,403.70$ & 4534474 \\
\hline $9 \mathrm{BW}$ & 3 & $548,177.80$ & 4514835 & $12 \mathrm{~B}$ & 1 & $563,741.60$ & 4528906 \\
\hline 9BW & 4 & $548,112.50$ & 4515083 & $12 \mathrm{~B}$ & 1 & $564,310.60$ & 4528443 \\
\hline 9BW & 4 & $548,104.30$ & 4514846 & $12 \mathrm{~B}$ & 2 & $563,579.60$ & 4528789 \\
\hline 9BW & 5 & $547,999.90$ & 4515072 & $12 \mathrm{~B}$ & 2 & $564,248.40$ & 4528255 \\
\hline 9BW & 5 & $548,018.60$ & 4514844 & $12 \mathrm{~B}$ & 3 & $563,514.90$ & 4528601 \\
\hline 10 & 1 & $547,167.60$ & 4516136 & $12 \mathrm{~B}$ & 3 & $564,156.00$ & 4528120 \\
\hline 10 & 1 & $547,237.60$ & 4516081 & $12 \mathrm{~B}$ & 4 & $563,212.70$ & 4528550 \\
\hline 10 & 2 & $547,136.30$ & 4516099 & $12 \mathrm{~B}$ & 4 & $564,024.10$ & 4527925 \\
\hline 10 & 2 & $547,206.80$ & 4516038 & $12 \mathrm{~B}$ & 5 & $563,080.10$ & 4528552 \\
\hline 10 & 3 & $547,102.40$ & 4516052 & $12 \mathrm{~B}$ & 5 & $563,812.10$ & 4527700 \\
\hline
\end{tabular}


Appendix D. Whooping Crane Roost Observations 


\section{Crane Roost Observation Data}

Information on Whooping Crane sightings along the Platte River during 1940-1974 are derived from records of the U.S. Fish and Wildlife Service, and information since 1975 is derived from the Cooperative Whooping Crane Migration Monitoring Project. The records are stored and maintained in a computer database and as paper hardcopies by the U.S. Fish and Wildlife Service, Ecological Services Field Office, at Grand Island, Nebraska.

A "sighting," as defined by the Cooperative Migration Monitoring Project to describe crane stopovers, is the observation of a single Whooping Crane or a group of Whooping Cranes that are migrating together through an area. Confirmed sightings in the same general area (within a reasonable distance of daily crane activities) along the Platte and within one to several days of another sighting are assumed to be the same bird/bird group, unless: (1) the number of birds differs, (2) the bird(s) constitute a bird/bird group in addition to those already known to be in the general area, or (3) the original birds were observed to migrate from the valley or are known to have moved to a different area of the valley. This assumption is necessary because individual cranes cannot be distinguished; very few birds are marked, and continuous surveillance of a crane or crane group using the study area is not possible.

Reported sightings are classified by the Service database managers as confirmed, probable, or unconfirmed, based on the definitions given in the Whooping Crane Recovery Plan.

\section{Confirmed Sighting}

Observation made by a state or federal biologist or officer or by other known qualified observer (trained ornithologist or birder with experience in identification of whooping cranes). A photograph may also be used to confirm sightings.

\section{Probable Sighting}

No confirmation made by state or federal biologist or officer or by other known qualified observer, yet details of the sighting seem to identify the birds as whooping cranes. To be classified as a probable sighting each of the following factors must be met: (1) location of sighting is within normal migration corridor and is an appropriate site for whooping cranes, (2) date of sighting is within period of migration, (3) accurate physical description, (4) number of birds is reasonable, (5) behavior of the birds does not eliminate whooping cranes, and (6) good probability that the observer would provide a reliable report.

\section{Unconfirmed Sighting}

Details of the sighting meet some, but not all of the six factors listed for a probable sighting.

The Service and cooperators have documented characteristics of Whooping Crane use sites along the Platte River since 1966. A total of 71 Whooping Crane roosting locations (for 50 different groups of birds) collected from 1966 through 2002 were used for the analyses in this report (Table D.1). Forty-six (46) of the observations included river depth profiles, and the first 21 of these were used (Carlson and others, 1990) to construct the C4R model's depth function (DF) criterion. The discharges (Q (cfs)) reported in Table D.1 were measured on the day that the crane transect was measured, or if that measurement was not available, we reported the discharge measured on the first day that the crane was observed.

Five primary records are made of crane stopover use-sites on the Platte River: (1) whooping crane sighting report forms used by the Cooperative Whooping Crane Migration Monitoring Program; (2) field site evaluation forms used by the Cooperative Whooping Crane Migration Monitoring Program; (3) a reporting form for channel profile measurements of use sites; (4) ground photographs of use sites; and (5) entry of use-locations into a GIS database (in preparation). The monitoring program and channel profile forms used by the Service are provided in Figure D.1, at the end of this Appendix. The data from field site evaluation forms are also entered into a digital database.

Field evaluation and measurements of use sites are obtained as soon as possible following crane departure. Channel profile data were obtained by placing a cross section through the channel use site, perpendicular to the river flow (Lingle and others, 1984). Units for cross section length were in feet and vertical measurements were to the nearest 0.1 foot. Whooping Crane riverine suitability information such as channel width, water width, and water depth characteristics are derived from channel profile measurements. 
These characteristics are applicable to the descriptions of Whooping Crane use-site on the Platte River. Information available for baseline parameters at crane use-sites, extracted from the Service's information on file at Grand Island includes:

1. Active channel width is measured perpendicular to the flow, from one permanent bank to another.

2. Water depths vary across the channel at each site, providing shallowly submerged sandbars and deeper channels around the sandbars (Faanes and others, 1992). Water depth is measured at 10 foot increments along the channel width. Transitions between water and bare sand are also measured.

3. Water width is the summed width of the channel profile cross section that is inundated by water. Water width data is obtained with the channel profiles measurements. 


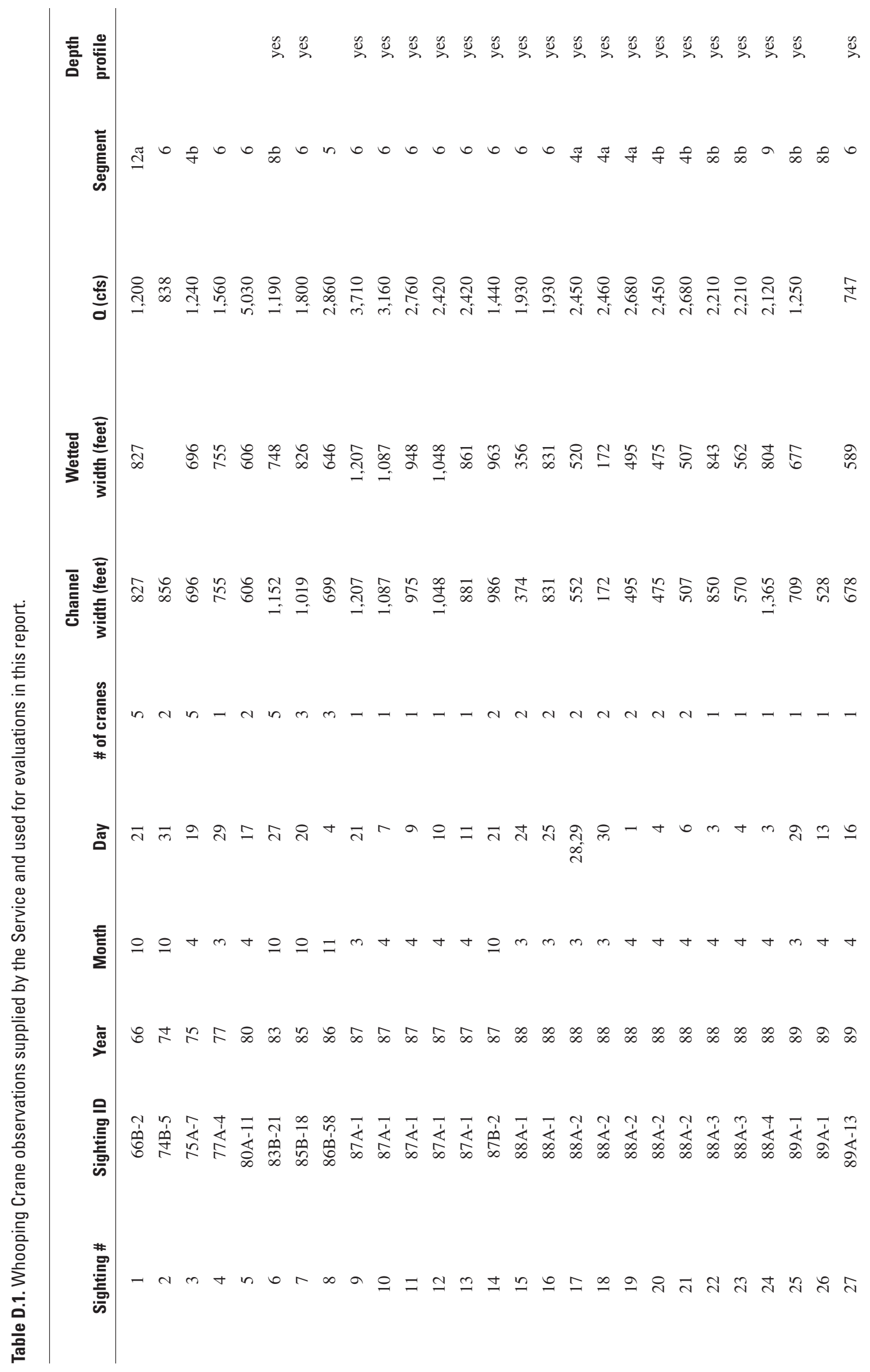




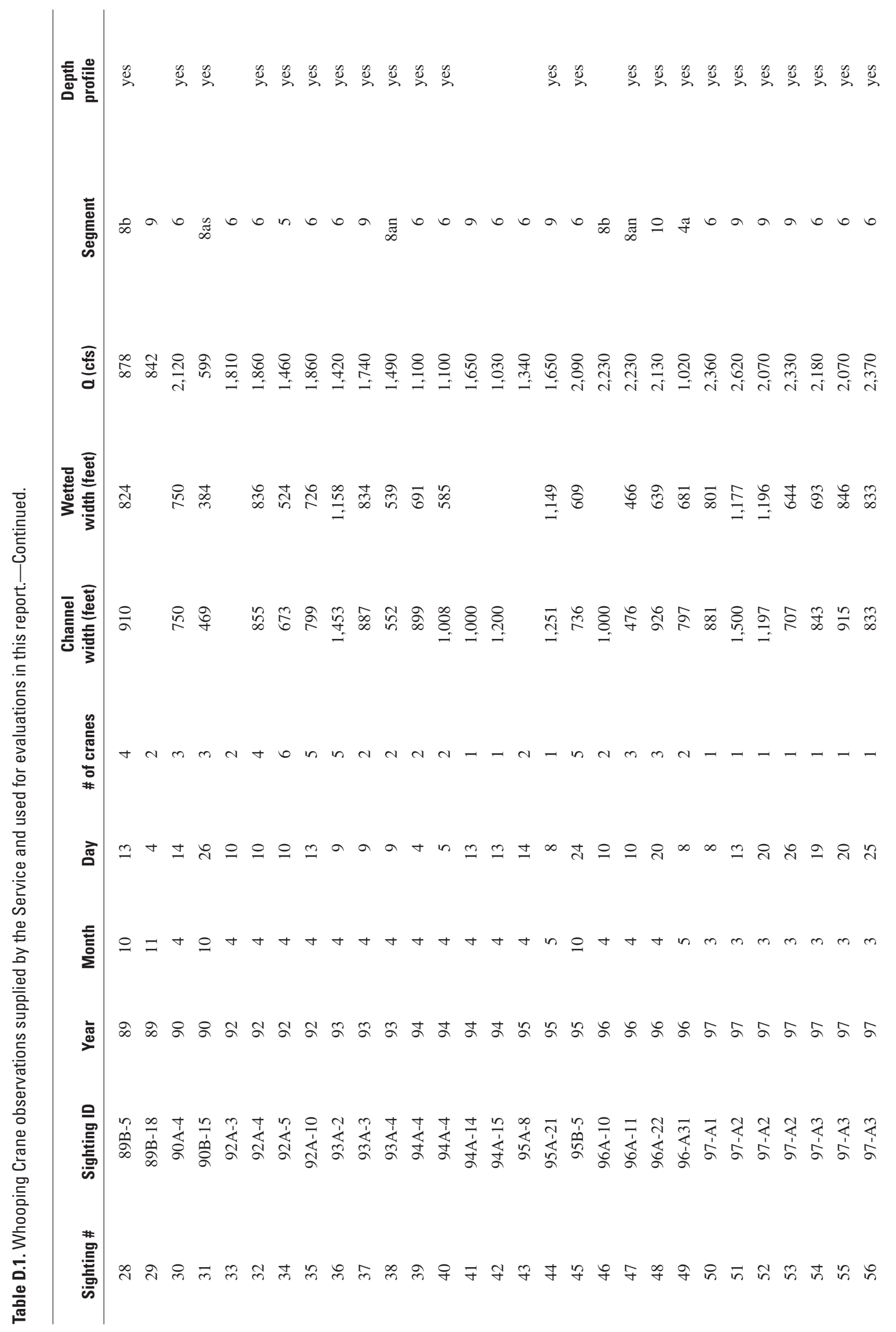




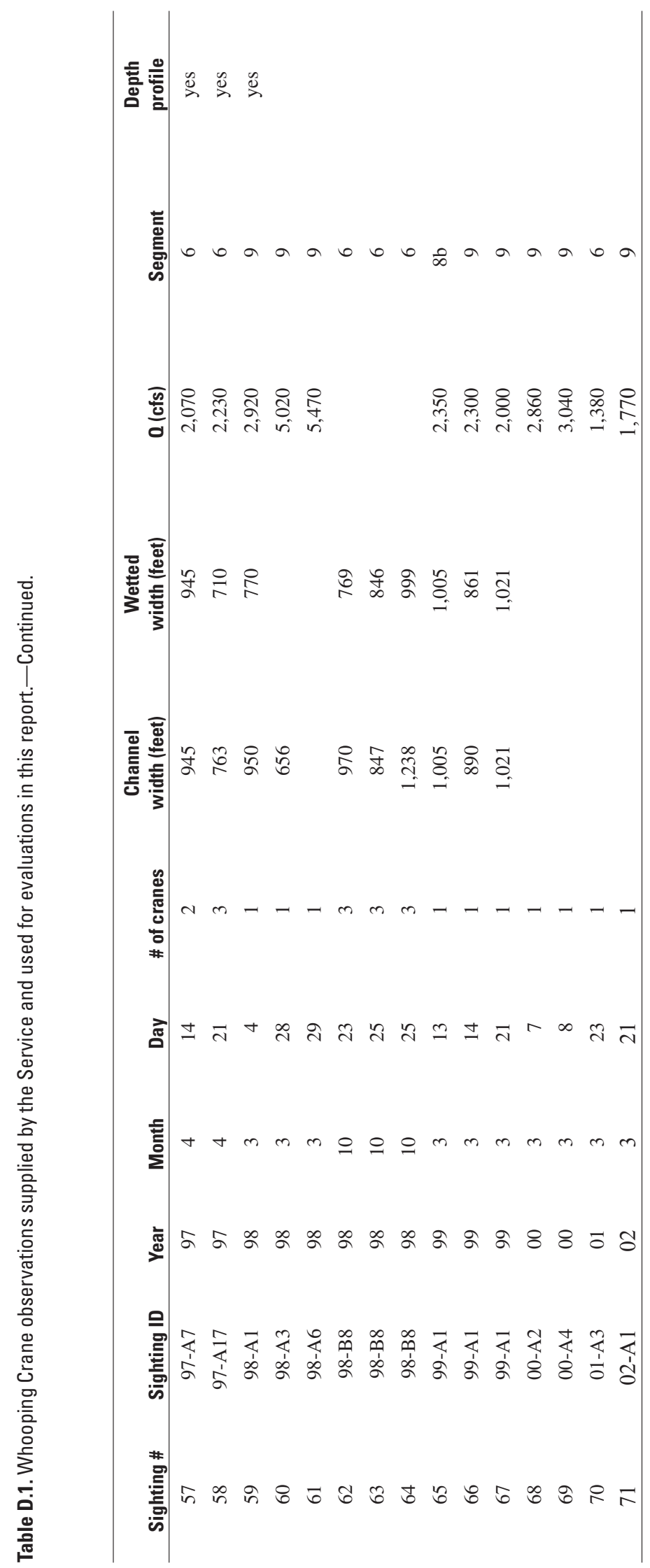




\section{Appendix D. Whooping Crane Roost Observations-Continued.}

\section{Whooping Crane Riverine Use-Site Channel Profile and Site Evaluation}

Observation Identification Number:

Date(s) of observed use:

Total number of adult cranes:

Total number of cranes with juvenile plumage:

Name and contact information of initial observer(s):

Contact information for person confirming observation:

Location:

C.O.E. river mile:

Date profile was measured:

Time:

Reconnaissance party and affiliation:

River discharge (cfs): Gage:

Does the channel carry total river flow? No Yes

Total water width (feet):

Active channel width (feet):

Percent wet (TWW/ACW):

Are exposed sandbars present in the vicinity? No Yes

If sandbars are present, describe height, distance, and location in relation to roost:

Upstream visibility (feet):

Downstream visibility (feet):

Height and density of bank vegetation:

Disturbance factors (describe feature and distance): 


\section{Appendix D. Whooping Crane Roost Observations-Continued.}

Riverine Use Site Evaluation-Continued.

Observation Identifcation Number:

Location:

Channel profile:

\begin{tabular}{|c|c|c|}
\hline Station (feet) & Depth (feet) & Comments \\
\hline & & \\
\hline & & \\
\hline & & \\
\hline & & \\
\hline & & \\
\hline & & \\
\hline & & \\
\hline & & \\
\hline & & \\
\hline & & \\
\hline & & \\
\hline & & \\
\hline & & \\
\hline & & \\
\hline & & \\
\hline & & \\
\hline & & \\
\hline & & \\
\hline & & \\
\hline & & \\
\hline & & \\
\hline & & \\
\hline & & \\
\hline & & \\
\hline & & \\
\hline & & \\
\hline & & \\
\hline & & \\
\hline & & \\
\hline & & \\
\hline
\end{tabular}




\section{Appendix D. Whooping Crane Roost Observations-Continued.}

C.O.E. river mile: Using the Corps of Engineers 1985 photo atlas for the Platte River or Department of the Interior GIS. Active channel width: Measurement of width of the channel used by the cranes. Active channel is the portion periodically inundated with a frequency to maintain an active channel bed.

Total water width: Sum of the total wetted width measured along the cross-channel transect.

Sandbars: Note the presence or absence of sandbars on the transect, and in the vicinity, upstream and downstream of the transect. Include height of the sandbar, vegetation on the bar, and location of the bars in the channel and in relation to location used by cranes.

Disturbance factors: Can include lights, noise, or human activity (houses, roads, railroad tracks).

Channel profile: Measure water depths at 10 foot intervals or where changes in channel profile occur on a transect perpendicular to the channel and through the roost. In comments, note the stations where prominent features occur, e.g., edge of water at sandbars and at bank; top of bank; and the stations the cranes were observed to use. 
98 Printed on recycled paper 